\title{
Regulation of prefrontal patterning and connectivity by retinoic acid
}

https://doi.org/10.1038/s41586-021-03953-x

Received: 31 December 2019

Accepted: 25 August 2021

Published online: 1 October 2021

Check for updates

\author{
Mikihito Shibata", ${ }^{1,2}$, Kartik Pattabiraman ${ }^{1,2,12}$, Belen Lorente-Galdos', David Andrijevic', \\ Suel-Kee Kim ${ }^{1}$, Navjot Kaur', Sydney K. Muchnik ${ }^{1,3}$, Xiaojun Xing ${ }^{1,4}$, Gabriel Santpere ${ }^{1,5}$, \\ Andre M. M. Sousa ${ }^{1,6,7}$ \& Nenad Sestan ${ }^{1,3,4,8,9,10,11 凶}$
}

The prefrontal cortex (PFC) and its connections with the mediodorsal thalamus are crucial for cognitive flexibility and working memory ${ }^{1}$ and are thought to be altered in disorders such as autism ${ }^{2,3}$ and schizophrenia ${ }^{4,5}$. Although developmental mechanisms that govern the regional patterning of the cerebral cortex have been characterized in rodents ${ }^{6-9}$, the mechanisms that underlie the development of PFCmediodorsal thalamus connectivity and the lateral expansion of the PFC with a distinct granular layer 4 in primates ${ }^{10,11}$ remain unknown. Here we report an anterior (frontal) to posterior (temporal), PFC-enriched gradient of retinoic acid, a signalling molecule that regulates neural development and function ${ }^{12-15}$, and we identify genes that are regulated by retinoic acid in the neocortex of humans and macaques at the early and middle stages of fetal development. We observed several potential sources of retinoic acid, including the expression and cortical expansion of retinoic-acid-synthesizing enzymes specifically in primates as compared to mice. Furthermore, retinoic acid signalling is largely confined to the prospective PFC by CYP26B1, a retinoic-acid-catabolizing enzyme, which is upregulated in the prospective motor cortex. Genetic deletions in mice revealed that retinoic acid signalling through the retinoic acid receptors RXRG and RARB, as well as CYP26B1-dependent catabolism, are involved in proper molecular patterning of prefrontal and motor areas, development of PFC-mediodorsal thalamus connectivity, intra-PFC dendritic spinogenesis and expression of the layer 4 marker RORB. Together, these findings show that retinoic acid signalling has a critical role in the development of the PFC and, potentially, in its evolutionary expansion.
The PFC reaches its greatest complexity in anthropoid primates (monkeys and apes), which appear to uniquely have many prefrontal areas that cover theentire anterior two-thirds of the frontal lobe and a well-defined granular layer $4^{10,11}$. Previous analyses revealed that the transcriptomic differences between neocortical areasaremost prominent in primates during themiddle stages of fetal development, corresponding to post-conception weeks (PCW) 13 to 24 (hereafter referred to as 'mid-fetal') ${ }^{16,17}$-a crucial period for neuronal specification and the initial assembly of neocortical neural circuits ${ }^{18}$. Thus, wehypothesized that themolecular processes that govern the development and evolutionary diversification of thePFC could be revealed by differential regional gene expression analysis of the primate mid-fetal neocortex.

\section{Fetal frontal upregulation of RA-related genes}

Using human BrainSpan RNA-sequencing (RNA-seq) data ${ }^{17}$, we screened for genes that are differentially upregulated in the mid-fetal frontal lobe. The mid-fetal data consisted of tissue-level samples ranging in age from PCW 16 to 22, which included four prospective PFC areas (medial, mPFC or MFC; orbital, oPFC or OFC; dorsolateral, dIPFC or DFC; and ventrolateral, vIPFC or VFC) and the primary motor cortex (M1C). Gene expression in these frontal areas was compared to areas within the parietal (primary sensory cortex, S1C; and inferior parietal cortex, IPC), occipital (primary visual cortex, V1C), and temporal lobes (primary auditory cortex, A1C; superior temporal cortex, STC; and inferior temporal cortex, ITC) (Fig. 1a, Extended Data Fig. 1a). We identified 190 protein-coding genes, using stringent criteria, that were specifically upregulated in at least one area within a lobe in comparison with areas from other lobes, including 125 in the frontal lobe, which were able to differentiate the four brain lobes and most areas within them (as observed by principal component analysis (PCA)) (Fig. 1b, Extended Data Fig. 1a, b). Moreover, the first principal component (PC1), which accounts for the highest variability present in the data,

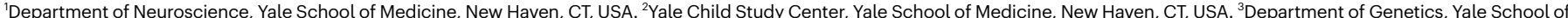

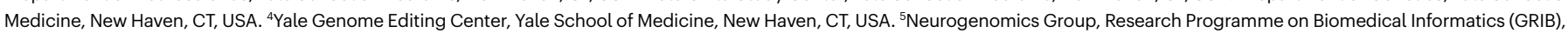

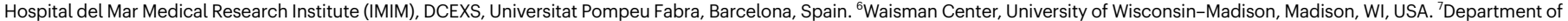
Neuroscience, University of Wisconsin-Madison, Madison, WI, USA. ${ }^{8}$ Department of Comparative Medicine, Yale School of Medicine, New Haven, CT, USA. ${ }^{9}$ Department of Psychiatry, Yale

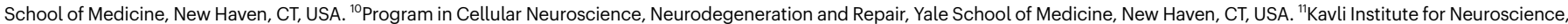
Yale University, New Haven, CT, USA. ${ }^{12}$ These authors contributed equally: Mikihito Shibata, Kartik Pattabiraman. ${ }^{凶}$ e-mail: nenad.sestan@yale.edu 

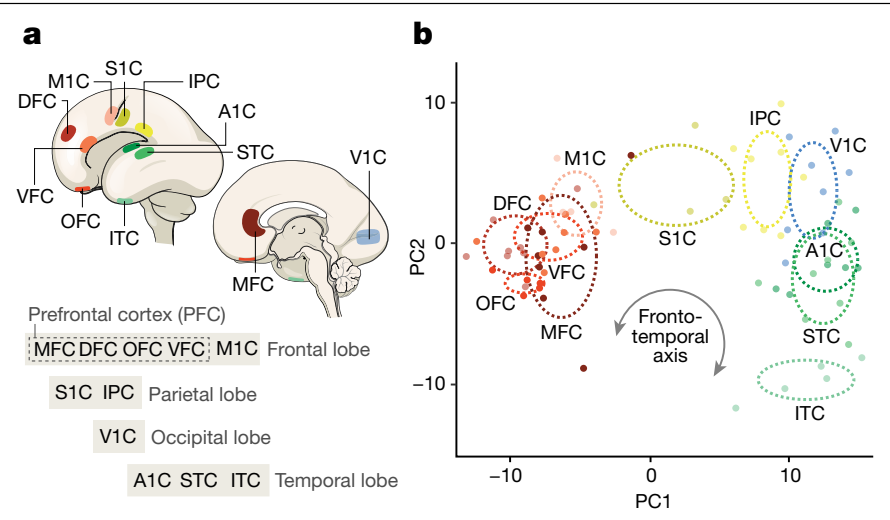

c
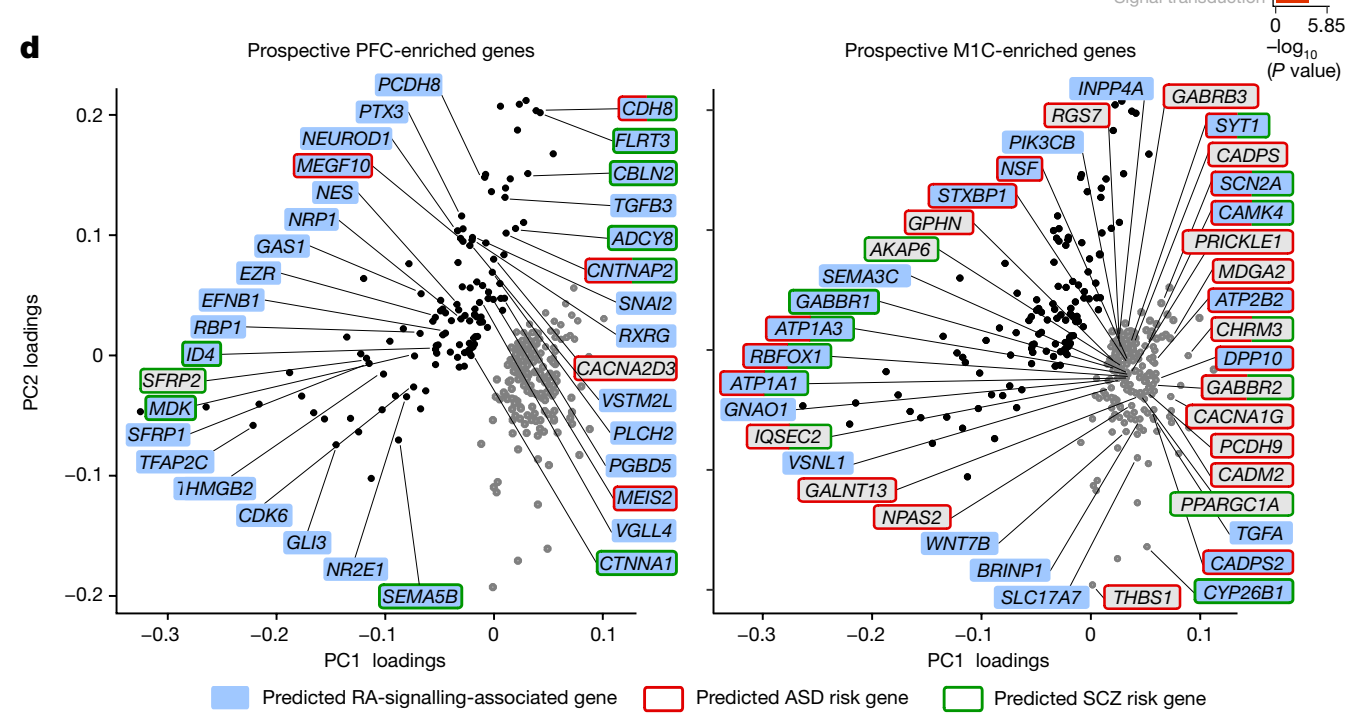

Fig. 1 | Predicted RA-signalling-associated genes are upregulated in human mid-fetal prospective frontal areas. a, Diagram of the analysed eleven areas of the human mid-fetal brain from the four lobes. b, PCA of genes specifically enriched in at least one area within a lobe. Ellipses are centred on the mean of the points of a given area and the size of the axes corresponds to their standard deviation on each component.c, GO terms associated with frontally

corresponded to the anterior-posterior or frontal-temporal axis (Fig. 1b). Gene Ontology (GO) analysis of the frontally upregulated genes identified an enrichment of genes associated with categories such as 'response to retinoic acid', 'synapse organization/assembly' and 'axon development/guidance' (Fig.1c, d, Supplementary Tables 1, 2 ), suggesting that these genes may have a role in frontal lobe patterning and circuit development. Many of the same frontal-lobe-enriched and retinoic acid (RA)-related genes were upregulated in the mid-fetal macaque frontal cortex using a PsychENCODE datase ${ }^{19}$ (Extended Data Fig. 1c, d). We also observed prominent frontal enrichment of many of the same genes during the early fetal period (Extended Data Figs.1, 2). Closer analysis of the spatiotemporal profile of the fetal frontally upregulated genes revealed the enrichment to be largely transient, with mainly conserved and some divergent expression patterns between human and macaque (Extended Data Fig. 4b).

Analysing only the RNA-seq data that encompassed the five mid-fetal frontal areas ${ }^{17}$, predicted RA-signalling-associated genes-such as $C B L N 2, R X R G, C D H 8, M E I S 2$ and $R B P 1$-were among the genes upregulated in the PFC compared to the M1C (Fig. 1d, Supplementary Table 2; see our accompanying study ${ }^{20}$ ), whereas the RA-degrading enzyme CYP26B1 was upregulated in the M1C (Fig. 1d), consistent with previous microarray-based findings ${ }^{21}$. We also identified multiple predicted autism spectrum disorder (ASD) (group 1-3; https://gene.sfari.org/) and schizophrenia (SCZ) (score of 3 or higher from http://szdb.org/) upregulated genes and their unadjusted $P$ value. Black text is used to highlight relevant $\mathrm{GO}$ terms. d, PC loadings with labelled genes being upregulated in the PFC (black dots) and M1C (grey dots). Genes highlighted in blue are associated with RA signalling and genes with red or green outlines are predicted ASD or SCZ risk genes, respectively. For reproducibility information, see Methods.

risk genes that were upregulated in the mid-fetal frontal cortex (Fig.1d, Supplementary Table 2).

\section{Mid-fetal PFC-enriched gradient of RA}

RA is a diffusible biologically active derivative of vitamin A that is involved in neural tube patterning, neurogenesis, cell differentiation and synaptic function ${ }^{12,13,22-27}$. Moreover, alterations in RA signalling have been implicated in the pathophysiology of $\mathrm{ASD}^{28-30}$ and $S C Z^{14,31,32}$. Given the enrichment of RA-related genes among the upregulated fetal frontal genes, we assessed the concentration of RA in different areas of the human and macaque mid-fetal neocortex, and in the neonatal mouse neocortex (approximately equivalent developmental ages), using an enzyme-linked immunosorbent assay (ELISA). We found that there was a PFC-enriched anterior-posterior gradient of RA concentration in the human and macaque mid-fetal neocortex, with homologous mPFC exhibiting the highest concentration within each species (Fig. 2). Overall, RA concentrations were significantly higher in prospective primate PFC areas compared to more posterior areas. Comparison across the three species identified higher concentrations of RA both in the MPFC and in all four PFC areas overall in humans as compared to the other two species (Fig. 2), as well as in macaques compared to mice (Fig. 2). Notably, the ITC-an association area within the temporal lobe that is thought to exhibit unique features and connectivity 


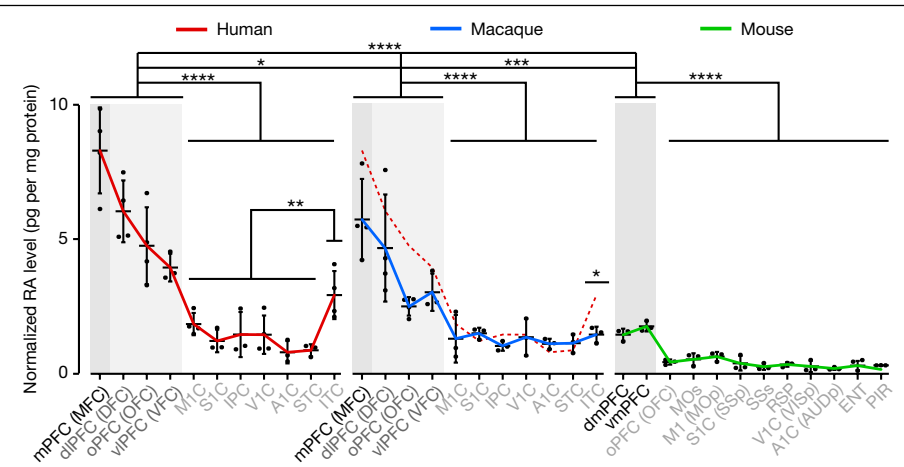

Fig. 2 | PFC-enriched anterior-posterior gradient of RA. RA concentrations in human (post-conception weeks (PCW) 16,18,19 and 21), macaque (four post-conception day (PCD) 110 brains) and mouse (four PD1brains) cortical areas ( $n=3-4$ for each sample area). One-way ANOVA with post-hoc Dunnett's adjustment or two-tailed unpaired $t$-test: ${ }^{* * * *} P<1 \times 10^{-4},{ }^{* * *} P=0.0005$ (macaque PFC versus mouse PFC), ${ }^{* *} P=0.005$ (human non-PFC minus ITC versus human ITC), ${ }^{*} P=0.01$ (human PFC versus macaque PFC), ${ }^{*} P=0.04$ (human ITC versus macaque ITC). Centre value, average; error bars, s.e.m. The dashed red line in the macaque graph represents the human RA concentrations. A single assay was done for each brain sample. dmPFC, dorsomedial PFC; ENT, entorhinal cortex; PIR, piriform cortex; RSP, retrosplenial cortex; SSs, secondary somatosensory area; VISp, primary visual cortext; vmPFC, ventromedial PFC.

in humans ${ }^{33}$-had a higher concentration of RA among non-frontal areas in humans (Fig. 2), with a two-fold increase in RA concentration in humans compared to macaques.

\section{Expanded RA synthesis in the primate cortex}

The observed enrichment and primate-specific lateral expansion of RA levels in the fetal PFC led us to systematically examine the spatiotemporal expression of RA-synthesizing enzymes (ALDH1A1-ALDH1A3) and RA-degrading enzymes (CYP26A1, CYP26B1 and CYP26C1 ${ }^{27,28}$ ) in human, macaque and mouse (for full descriptions, see Supplementary Results). In brief, we observed several potential sources of RA in the fetal cortex, including conserved expression of ALDH1A1 in the meninges and midbrain axons, and ALDH1A3 in the $\mathrm{mPFC}^{26}$. We also observed primate-specific expression of ALDH1A1 in astrocytes and frontal subplate neurons, and lateral frontal expression of $A L D H 1 A 1$ and $A L D H 1 A 3$ (Extended Data Fig. 6). These primate-specific expression patterns were complementary to the increase in and lateral expansion of RA in the mid-and late-fetal primate PFC and the lateral extension of PFC in anthropoid primates ${ }^{10,11}$.

\section{Frontal RA signalling is mediated by RARB and RXRG}

Given the fetal frontal cortical upregulation of both RA and RA synthesizing enzymes, we further assessed the expression of RA-dependent receptors and RA-responsive downstream genes in the developing human, macaque and mouse cortex. In addition to $R X R G$, which is upregulated in the human mid-fetal PFC ${ }^{21}$ (Extended Data Fig 1c), several genes that encode RA receptors were also detected in the developing human, macaque and mouse cortex or cultured human primary mid-fetal cortical neurons (Extended Data Figs. 5, 8, 9, 15b). Of these, only RARB and RXRG exhibited a higher anterior to lower posterior gradient of expression (Extended Data Figs. 8, 9a).

The RARB-RXRG heterodimer has previously been shown to mediate RA signalling in the adult mouse cortex and striatum, and is required for learning, locomotion and dopamine signalling ${ }^{34,35}$. To assess whether RARB and RXRG are required for RA signalling activity in the developing mouse frontal cortex, we generated constitutive Rarb and
Rxrg double-knockout (dKO) mice (Extended Data Fig. 9d), whichconsistent with previous findings ${ }^{34,35}$-are viable. Using a RARE-lacZ reporter line ${ }^{36}$ in which lac $Z$ is under the transcriptional control of an RA response element, we identified a significant reduction of RA signalling in the $\mathrm{mPFC}$ of $\mathrm{dKO}$ mice at post-natal day (PD) 0 compared to control mice (Fig. 3a, Extended Data Fig. 10a). In addition, we found a less extensive reduction of RA signalling in the anterior cingulate area (ACA) and retrosplenial area (Extended Data Fig. 10a). Furthermore, expression of frontally enriched RA-regulated genes, $\mathrm{Cbln} 2^{20}$ and Meis2, which are induced by RA in human and chimpanzee cerebral organoids and repressed by RA receptor antagonists in human cortical neurons (Fig. 1d, Extended Data Figs. 11b, 15d), was reduced in the dKO mice (Extended Data Fig. 10b). Together, these findings indicate that RARB and RXRG mediate RA signalling in the developing mouse mPFC.

\section{RARB and RXRG regulate frontal connectivity}

To understand the functional importance of RA signalling through the RARB-RXRG heterodimer in the developing cortex, we performed RNA-seq analysis of different regions of the PD 0 mouse frontal cortex (mPFC; secondary motor cortex (MOs) and the adjacent parts of the primary motor cortex (MOp); and OFC) microdissected from dKO and wild-type littermates. We identified 4,768 differentially expressed protein-coding genes between the two genotypes in at least one of the areas, with the highest number of differentially expressed genes in the mPFC (Extended Data Fig. 12a, Supplementary Table 3). PCA based on the expression of these differentially expressed genes separated the wild-type and dKO mice along PC1, with the MPFC showing the greatest distance between wild type and $\mathrm{dKO}$, further supporting the notion that the $\mathrm{MPFC}$ is most affected by the reduction in RA signalling (Fig. 3b).

The GO enrichment analysis revealed that terms associated with genes that are overexpressed in the wild-type compared to the dKO frontal cortex were highly related to the process of synaptogenesis and cellular components related to synapses and axons, whereas the genes that are overexpressed in the $\mathrm{dKO}$ were related to the regulation of the cell cycle (Fig. 3c, Extended Data Fig. 12c, d). In addition, when analysing differentially expressed genes in individual regions of the frontal cortex, only genes overexpressed exclusively in the wild-type mPFC were associated with the cellular components, axons and synapses (Extended Data Fig. 12c, d). Several of the genes overexpressed in wildtype mice that are related to axon guidance and synapse development exhibited an anterior enrichment in wild-type neonatal mouse cortex (Extended Data Fig. 13a, Supplementary Table 4). We also observed a significant enrichment of homologous genes specifically upregulated in the human mid-fetal frontal lobe among the genes that were downregulated exclusively in the frontal cortex and $\mathrm{mPFC}$ of the dKO mice (Extended Data Fig. 12b, e). Overall, these results suggest a possible role for RA signalling in the regulation of synaptogenesis and axon development, specifically in the mPFC.

We analysed the role of RA in synaptogenesis by quantifying synaptic puncta in multiple regions of the PD 0 cortex in dKO mice compared to wild-type littermate controls. We identified a significant reduction in DLG4 (also known as PSD95)-positive excitatory post-synaptic densities in the dKO mPFC and MOs compared to wild-type, but not in the OFC, primary motor area (MOp) and primary somatosensory area (SSp) (Fig.3d, Extended Data Fig. 13b). Similarly, we identified a significant decrease in synaptophysin (SYP) and DLG4 co-immunolabelled puncta in deep layers of the MPFC (Extended Data Fig 13c) and a decrease in number of all and mushroom dendritic spines in mPFC pyramidal neurons labelled by the retrograde viral tracer $A$ AVrg-Cag-Gfp (Extended Data Fig. 14e). Of note, there was no difference observed in the dendritic complexity of mPFC upper-layer neurons (Extended Data Fig. 14e). In addition, treating human primary cortical neurons with RA induced the expression of DLG4 (PSD95) mRNA, and DLG4 (PSD95) and SYP co-immunolabelled synaptic puncta, whereas applying the RA receptor inhibitor AGN193109 


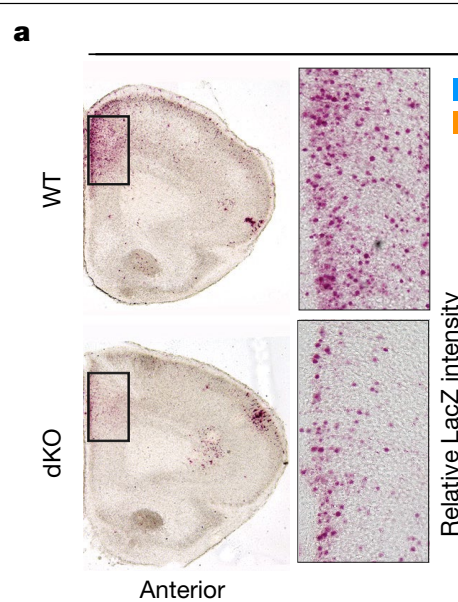

RARE-lacZ
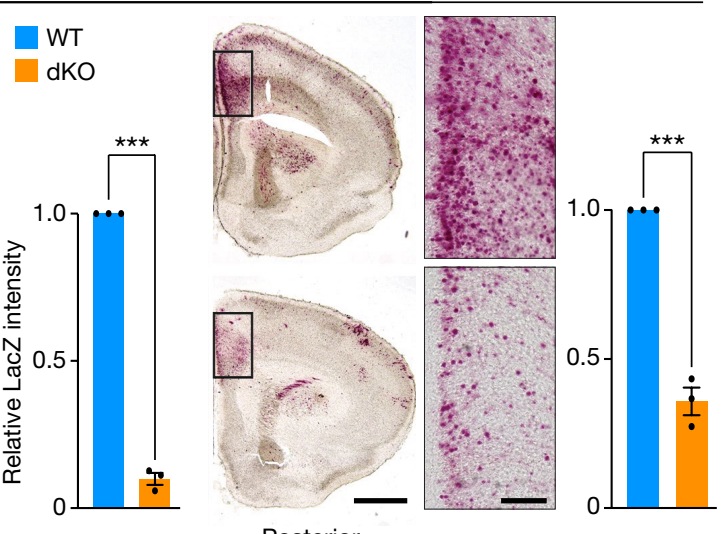

Posterior b
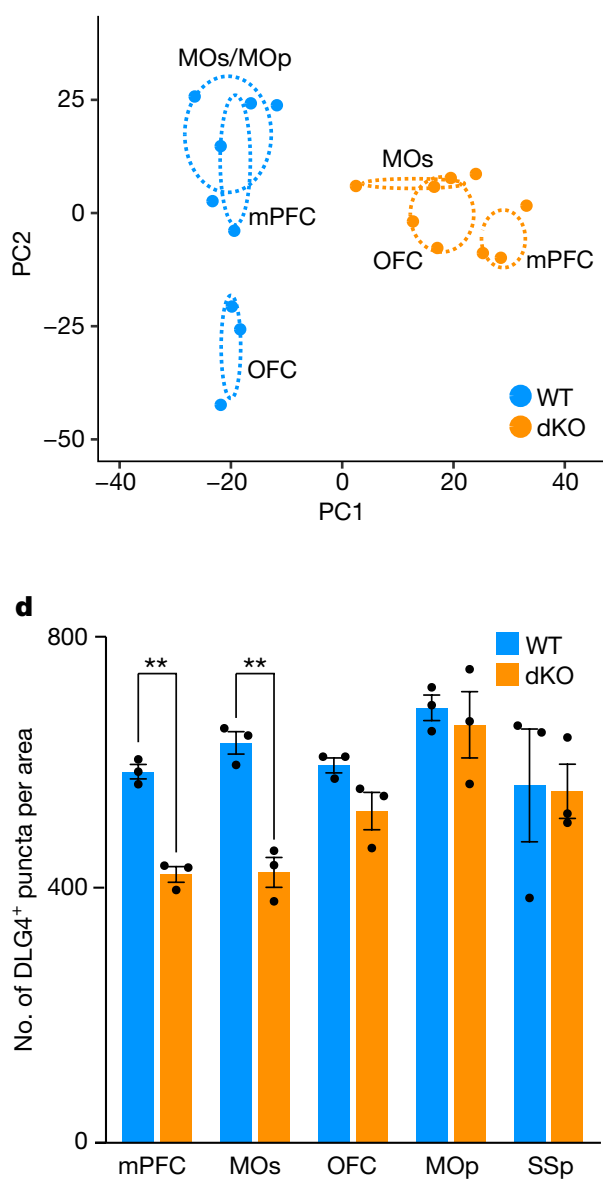

c

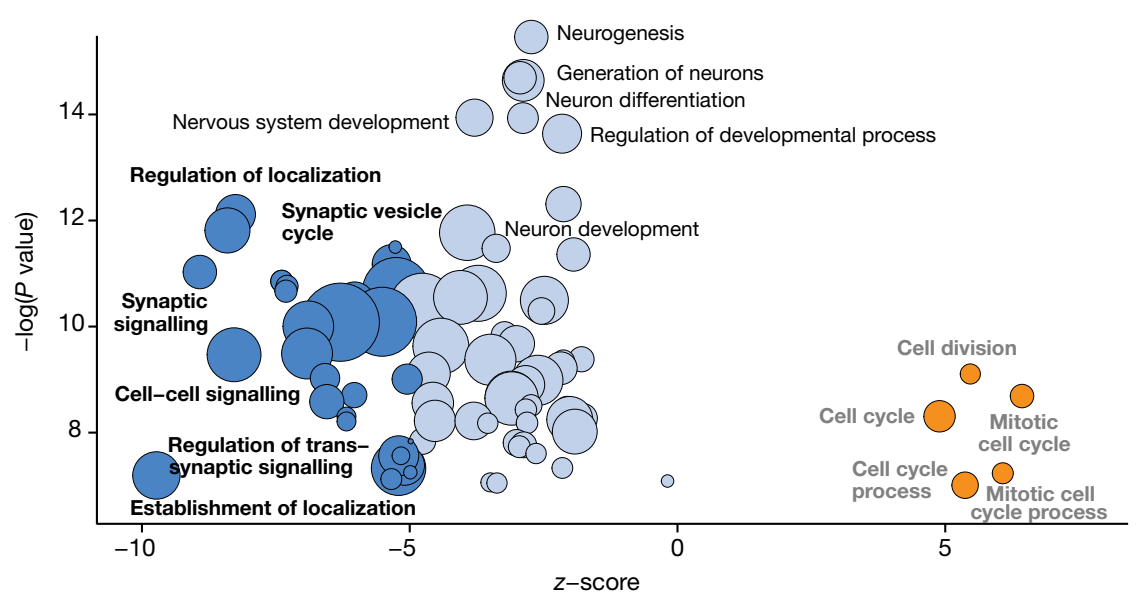

showing their $z$-score and unadjusted $P$ values. The $z$-score represents the proportion of upregulated versus downregulated genes in the dKO compared to the WT that are associated with each GO term. Dark blue, $z$-score $<-5$; light blue, $z$-score -5 to 0 ; orange, $z$-score $>0$. The size of the bubbles is proportional to the total number of differentially expressed genes associated with the given GO term. Black text is used to highlight relevant GO terms. d, Quantification of excitatory synapses marked by DLG4 (PSD95) in the mPFC, MOs, OFC, MOp and SSp regions of PD 0 WT and dKO mice brains. Two-tailed Student's $t$-test: ${ }^{* *} P=6 \times 10^{-4}$ (mPFC); $2 \times 10^{-3}$ (MOs). Centre value, average; error bars, s.e.m. ( $n=3$ per genotype). For reproducibility information, see Methods.

had the opposite effect (Extended Data Fig. 15e-j). Similarly, treatment with RA-soaked beads induced the expression of DLG4 (PSD95) in both human and chimpanzee cerebral organoids (Extended Data Fig. 11b,c). We assessed the role of RA signalling in regulating the expression of the gene encoding synaptic organizer CBLN2 and local connectivity in our accompanying study ${ }^{20}$.

We investigated the role of RA signalling in long-range connections from the mPFC using diffusion tensor imaging (DTI). We identified a reduction in long-range connections between the $\mathrm{MPFC}$ and thalamus in dKO compared to wild-type mice at PD 5 (Fig. 4a, b). There was no observable difference in connections between the left and right $\mathrm{mPFC}$ at PD 5 (Fig. 4a, c). Owing to the limitations of the technique and the developmental stage, we were unable to study the connections between the mPFC and striatum or basolateral amygdala (BLA) using DTI. Anterograde lipophilic axon-tracing experiments starting from the $\mathrm{mPFC}$ or the mediodorsal thalamus (MD) at PD 21 similarly identified a reciprocal reduction in PFC-MD connectivity, as well as a reduction in the number of fibres in the internal capsule (Fig. 4d, e, Extended Data Fig. 13d). To more fully examine $\mathrm{MPFC}$ connectivity in $\mathrm{NKO}$ mice, we used a retrograde viral tracer to study inputs into the PD $30 \mathrm{mPFC}$. We identified reduced inputs from the MD and anterior insula in $\mathrm{dKO}$ mice, whereas no obvious changes were identified in inputs from the contralateral mPFC, MOs or MOp, internal capsule, claustrum, piriform cortex, amygdala and ventral hippocampus (Extended Data Fig. 14a-d). Finally, we assessed for changes in dopaminergic innervation of the mPFC and found no significant difference between wild type and dKO (Extended Data Fig. 16f).

Although there is reduced RA activity in the outer shell of the striatum in the dKO brain (Extended Data Fig. 10a), we found that fibres traverse through the medial aspect of the striatum, suggesting that alterations in RA signalling in the lateral striatum do not affect the guidance of reciprocal $\mathrm{mPFC}-\mathrm{MD}$ connectivity. RA signalling has previously been implicated in thalamic development ${ }^{37}$, so we assessed whether other thalamocortical connections were altered in the dKO mice at PD 5 using DTI. There was no observable difference in thalamocortical connectivity with the MOp, primary auditory area (AUDp) or SSp (Fig. 4a, c, Extended Data Fig. 16b) or in the formation of barrel fields in the SSp (Extended Data Fig.16c). Given that Aldh1a3 is expressed in the $\mathrm{ACA}^{26}$, we also examined whether thalamocortical innervation was altered in 

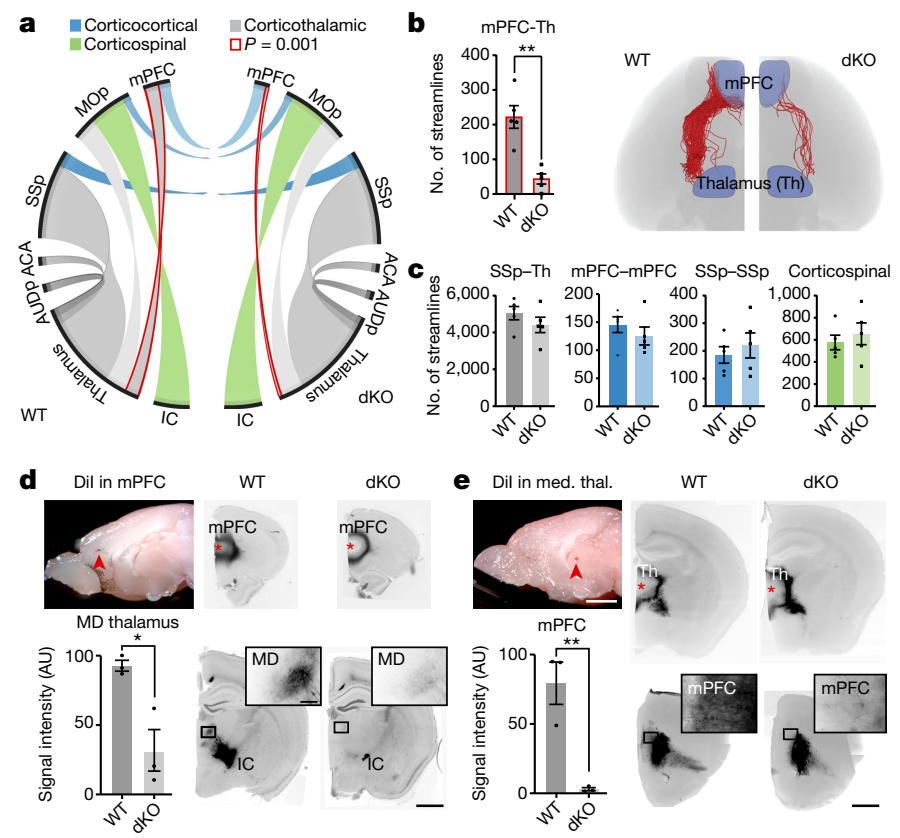

Fig. 4 | Altered mPFC-MD connectivity in mice lacking Rarb and $R x r g$. a, Representation of the number of streamlines generated as a connectivity measurement between the cortical areas, thalamus and internal capsule (IC) at PD 5 using DTI. b, Visualization and quantification of streamlines between the mPFC and the thalamus in WT and dKO brains. Two-tailed unpaired $t$-test: ${ }^{* *} P=1 \times 10^{-3}$. Centre value, average; error bars, s.e.m. $(n=5$ per genotype).c, Quantification of select corticothalamic, corticocortical and corticospinal streamlines. Two-tailed unpaired $t$-test: $P=0.2(\mathrm{SSp}-\mathrm{Th}$ ), 0.3 (mPFC-mPFC), 0.5 (SSp-SSp) and 0.5 (corticospinal). Error bars, s.e.m. $(n=5)$. d, e, Dil placement in the MPFC (d) and medial thalamus (e) in WT and dKO brains at PD 21 with labelled processes in the MD (d; inset), and $\mathrm{mPFC}$ (e; inset). Two-tailed Student's $t$-test: ${ }^{*} P=9 \times 10^{-3},{ }^{* *} P=3 \times 10^{-3}$. Centre value, average; error bars, s.e.m. ( $n=3$ per genotype). AU, arbitrary units. Scale bars, $1 \mathrm{~mm} ; 100 \mu \mathrm{m}$ (inset).

the ACA and found no difference in the number of streamlines (Fig. 4a, Extended Data Fig. 16b). The corticospinal tract (CST) and connections across the corpus callosum between the left and right mPFC, SSp and MOp in the dKO showed no difference in the number of streamlines in dKO compared to wild type (Fig. 4a, c, Extended Data Fig. 16b). Furthermore, scalar indexes, which describe the microstructural integrity of white matter, were similar between wild-type and dKO mice in the corpus callosum, anterior commissure and internal capsule (Extended Data Fig. 16a). In addition, the width of the CST at PD 30 was slightly increased in the dKO mice (Extended Data Fig. 16d). The reduction of connections between the MPFC and thalamus was not due to cell death in the MPFC (Extended Data Fig. 16e). In summary, deletion of Rarb and Rxrg leads to a reduction of RA signalling specifically in the $\mathrm{mPFC}$, as well as a selective reduction of reciprocal mPFC-MD connectivity.

RA signalling has previously been shown to be involved in the regulation of proliferation, cell cycle timing and cortical laminar development ${ }^{22-24}$, which could underlie our findings. Thus, we assessed whether the constitutive deletion of Rarb and $R x r g$ leads to changes in cortical size and laminar organization. The volume of the brain, cortex and frontal cortex at PD 5 were not significantly different when measured by magnetic resonance imaging (MRI) (Extended Data Fig. 17a). We also quantified the number of neurons using the upper-layer markers CUX1 and POU3F2 (BRN2), layer 4 marker RORB, and deep-layer markers BCL11B (CTIP2) and TBR1, and found no difference between wild type and dKO in the MPFC, MOs or visual cortex (Extended Data Fig. 17b). We did find a modest reduction in RORB-labelled neurons in the motor cortex (Extended Data Fig. 17b). Of note, the mouse mPFC lacks a granular layer 4 and had minimal RORB-labelled cells.

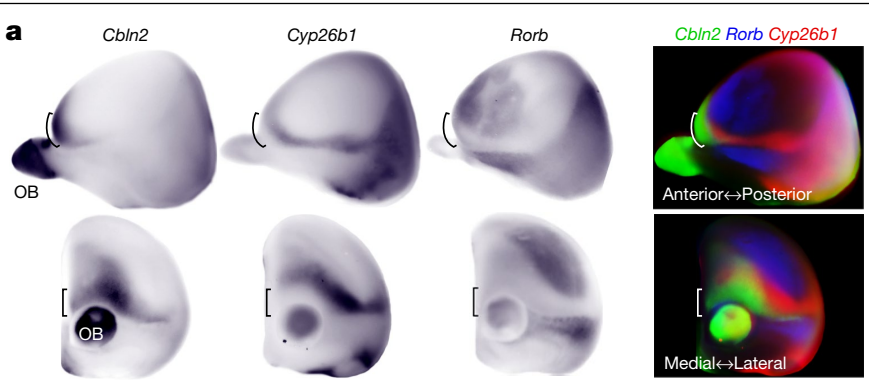

b Cyp26b1

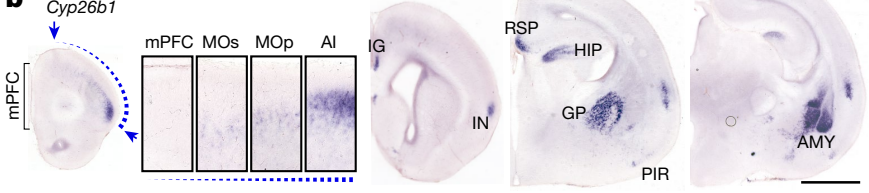

c

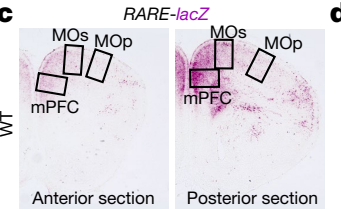

d Dil in med. thal.
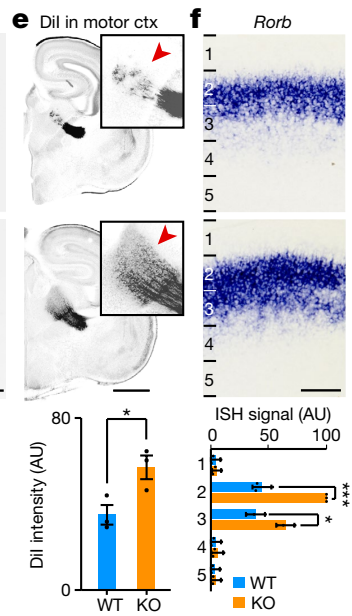

Fig. 5 | Increased RA signalling promotes mPFC-medial thalamic connectivity. a, Lateral (top) and coronal (bottom) views of whole-mount in situ hybridization (ISH) of the PD 0 mouse cortex with pseudo-colour merge of Cbln2, Cyp26b1 and Rorb. OB, olfactory bulb. b, Expression of Cyp26b1 in the PD 0 mouse brain using ISH. Cyp26b1 expression shows a gradient from the insula (AI) to the MOs (insets). Arrows indicate Cyp26b1 expression at the insula and MOs. Scale bar, $200 \mu \mathrm{m}$. AMY, amygdala; GP, globus pallidus; HIP, hippocampus; IG, indusium griseum; IN, insula.c, $\beta$-Galactosidase histochemical staining of the mPFC of Cyp26b1 $1^{++}(\mathrm{WT}) ; R A R E-l a c Z$ and Cyp26b1 ${ }^{-1-}(\mathrm{KO}) ;$ RARE-lacZ mouse brains at PCD 18. Relative signal intensity was quantified in the boxed area compared with anterior mPFC expression (mPFC, MOs and MOp). Two-tailed Student's $t$-test (WT versus Cyp26b1 KO): ${ }^{* * *} P=1 \times 10^{-4}, 6 \times 10^{-5}$ (mPFC, MOp), ${ }^{* *} P=4 \times 10^{-4} ; 1 \times 10^{-3}$ (MOs, MOp), ${ }^{*} P=4 \times 10^{-3}, 2 \times 10^{-2}$ (mPFC, MOp). Error bars, s.e.m. ( $n=3$ per genotype). d, e, Representative images and quantification of Dil labelling in PCD 18 frontal MOp/MOs (d) and medial thalamus (e) after Dil was placed in the MD thalamus or mPFC of WT and Cyp26b1 KO brains. Two-tailed Student's $t$-test: ${ }^{* * *} P=4 \times 10^{-4},{ }^{*} P=0.03$. Error bars, s.e.m. ( $n=3$ per genotype and condition). Scale bars: $200 \mu \mathrm{m} . \mathbf{f}$, Representative image and quantification of expression of Rorb in WT and KO mice in PCD 18 primordial motor cortex divided into 5 bins (1-5). Two-tailed Student's $t$-test (WT versus Cyp26b1 KO): ${ }^{* * *} P=2 \times 10^{-4}$, ${ }^{*} P=0.01$. Centre value, average; error bars, s.e.m. $(n=3$ per genotype).

\section{Ectopic RA expands MD connectivity}

MD innervation of the primate frontal cortex is expanded laterally compared to rodents, with the primate PFC areas having a more prominent layer $4^{10,11}$. Thus, we investigated whether the expansion of RA signalling is sufficient to increase and laterally extend MD innervation and alter layer 4 development in the mouse neonatal frontal cortex by genetically deleting the RA-degrading enzyme CYP26B1 in mice 
(Extended Data Fig.18a). Consistent with previous findings in humans ${ }^{21}$ and mice ${ }^{24}$, whole-mount and serial tissue section in situ hybridization revealed that $C y p 26 b 1$ is upregulated in the prospective motor cortex (MOs and MOp) and anterior insula compared to the MPFC and OFC, defined by high expression of Cbln2, and SSp, defined by Rorb (Fig. 5a, b, Extended Data Fig. 18b). Although mice with constitutive deletion of Cyp26b1 died perinatally, it has previously been shown that genetic deletion of Cyp26b1 in mice resulted in the expansion of RA signalling in multiple organs prenatally ${ }^{38}$ and post-natally in the mouse $\mathrm{mPFC}^{24}$. To investigate the possible role of CYP26B1 in restricting RA signalling to the mouse medial frontal cortex, we generated Cyp $26 b 1 \mathrm{KO}$ mice that also contained the RARE-lacZ transgene. The lack of $C y p 26 b 1$ resulted in the spreading of RA signalling dorsolaterally toward the MOs and MOp regions of the RARE-lacZ reporter mouse line at post-conceptional day (PCD) 18 (Fig. 5c). This increase was not robustly observed in more posterior regions (Extended Data Fig. 18c).

Next, we used the Cyp26b1 KO mice to study the effects of the expansion of RA signalling into the dorsolateral frontal cortex by inserting a crystal of $1,1^{\prime}$-dioctadecyl-3,3,3',3'-tetramethylindocarbocyanine perchlorate (Dil) into the medial thalamus of fixed post-mortem wild-type and KO brains, which were collected at PCD 18 owing to the perinatal lethality of the $\mathrm{KO}$ mice. Histological analysis revealed that wild-type littermates had occasional thalamocortical axons within the medial and dorsolateral frontal white matter and cortex at this age. By contrast, $\mathrm{KO}$ mice showed precocious and robust innervation of both the medial and the dorsolateral frontal cortex by the axons originating from the medial thalamus (Fig. 5d, Extended Data Fig. 19b). When Dil was placed in the motor cortex, KO mice showed an increased signal in the developing thalamus (Fig. 5e, Extended Data Fig. 19c). We also observed a moderately enlarged frontal cortex with grossly normal cytoarchitecture of the cortical wall and cortical plate in the analysed areas of the Cyp26b1 KO cortex (Extended Data Fig. 20a, b).

Furthermore, we observed an upregulation and expansion in the laminar expression of the layer 4 marker Rorb in the motor cortex of neonatal Cyp26b1 KO mice (Fig. 5f, Extended Data Fig. 19a) and after misexpression of Aldh1a3 in the dorsolateral fronto-parietal cortex using in utero electroporation (Extended Data Fig. 20c). In summary, we identified that ectopic RA signalling in the perinatal mouse frontal cortex leads to the expansion of medial thalamocortical innervation as well as the increased laminar expression of Rorb, which are both characteristics of the lateral granular PFC in anthropoid primates ${ }^{10,11}$.

\section{Conclusions}

We have shown here that RA signalling is required for proper prefrontal gene expression, spinogenesis and long-range connectivity. We propose that the early- and mid-fetal cortical expansion of RA signalling underlies the lateral expansion of PFC areas and MD innervation in primates. As the expansion of the prefrontal and temporal association areas has been proposed to be one of the evolutionary underpinnings of complex $\operatorname{cognition}^{9-11}$, it will be important to explore whether RA signalling has a broader role in developmental specification and the expansion of association areas, as well as in disorders affecting cognition (see Supplementary Discussion for a detailed discussion).

\section{Online content}

Any methods, additional references, Nature Research reporting summaries, source data, extended data, supplementary information, acknowledgements, peer review information; details of author contributions and competing interests; and statements of data and code availability are available at https://doi.org/10.1038/s41586-021-03953-x.
Fuster, J. M. The Prefrontal Cortex (Elsevier, 2015).

2. Amaral, D. G. et al. Neuroanatomy of autism. Trends Neurosci. 31, 137-145 (2008).

3. Willsey, A. J. et al. Coexpression networks implicate human midfetal deep cortical projection neurons in the pathogenesis of autism. Cell 155, 997-1007 (2013).

4. Tan, H.-Y. et al. Dysfunctional and compensatory prefrontal cortical systems, genes and the pathogenesis of schizophrenia. Cereb. Cortex 17, i171-i181 (2007).

5. Giraldo-Chica, M. et al. Prefrontal-thalamic anatomical connectivity and executive cognitive function in schizophrenia. Biol. Psychiatry 83, 509-517 (2018).

6. Fukuchi-Shimogori, T. \& Grove, E. A. Neocortex patterning by the secreted signaling molecule FGF8. Science 294, 1071-1074 (2001).

7. Cholfin, J. A. \& Rubenstein, J. L. R. Frontal cortex subdivision patterning is coordinately regulated by Fgf8, Fgf17, and Emx2. J. Comp. Neurol. 509, 144-155 (2008).

8. O'Leary, D. D. \& Sahara, S. Genetic regulation of arealization of the neocortex. Curr. Opin. Neurobiol. 18, 90-100 (2008).

9. Geschwind, D. H. \& Rakic, P. Cortical evolution: judge the brain by its cover. Neuron $\mathbf{8 0}$, 633-647 (2013).

10. Preuss, T. M. Do rats have prefrontal cortex? The Rose-Woolsey-Akert program reconsidered. J. Cogn. Neurosci. 7, 1-24 (1995).

11. Petrides, M. et al. The prefrontal cortex: comparative architectonic organization in the human and the macaque monkey brains. Cortex 48, 46-57 (2012).

12. Durston, A. J. et al. Retinoic acid causes an anteroposterior transformation in the developing central nervous system. Nature 340, 140-144 (1989).

13. Marshall, H. et al. Retinoic acid alters hindbrain Hox code and induces transformation of rhombomeres 2/3 into a 4/5 identity. Nature 360, 737-741 (1992).

14. Lamantia, A. S. Forebrain induction, retinoic acid, and vulnerability to schizophrenia: insights from molecular and genetic analysis in developing mice. Biol. Psychiatry 46, 19-30 (1999).

15. Maden, M. Retinoic acid in the development, regeneration and maintenance of the nervous system. Nat. Rev. Neurosci. 8, 755-765 (2007).

16. Pletikos, M. et al. Temporal specification and bilaterality of human neocortical topographic gene expression. Neuron 81, 321-332 (2014).

17. Li, M. et al. Integrative functional genomic analysis of human brain development and neuropsychiatric risks. Science 362, eaat7615 (2018).

18. Silbereis, J. C. et al. The cellular and molecular landscapes of the developing human central nervous system. Neuron 89, 248-268 (2016).

19. Zhu, Y. et al. Spatiotemporal transcriptomic divergence across human and macaque brain development. Science 362, eaat8077 (2018).

20. Shibata M. et al. Hominini-specific regulation of CBLN2 increases prefrontal spinogenesis. Nature https://doi.org/10.1038/s41586-021-03952-y (2021).

21. Johnson, M. B. et al. Functional and evolutionary insights into human brain development through global transcriptome analysis. Neuron 62, 494-509 (2009).

22. Siegenthaler, J. A. et al. Retinoic acid from the meninges regulates cortical neuron generation. Cell 139, 597-609 (2009).

23. Haushalter, C. et al. Retinoic acid controls early neurogenesis in the developing mouse cerebral cortex. Dev. Biol. 430, 129-141 (2017).

24. Larsen, R. et al. The thalamus regulates retinoic acid signaling and development of parvalbumin interneurons in postnatal mouse prefrontal cortex. eNeuro 6 , ENEURO.0018-19.2019 (2019).

25. Aoto, J. et al. Synaptic signaling by all-trans retinoic acid in homeostatic synaptic plasticity. Neuron 60, 308-320 (2008).

26. Smith, D. et al. Retinoic acid synthesis for the developing telencephalon. Cereb. Cortex 11, 894-905 (2001)

27. Dmetrichuk, J. M. et al. Retinoic acid induces neurite outgrowth and growth cone turning in invertebrate neurons. Dev. Biol. 294, 39-49 (2006)

28. Moreno-Ramos, O. A. et al. Whole-exome sequencing in a south American cohort links ALDH1A3, FOXN1 and retinoic acid regulation pathways to autism spectrum disorders. PLOS ONE 10, e0135927 (2015).

29. $\mathrm{Xu}, \mathrm{X}$. et al. Excessive UBE3A dosage impairs retinoic acid signaling and synaptic plasticity in autism spectrum disorders. Cell Res. 28, 48-68 (2018).

30. Kumar, S. et al. Impaired neurodevelopmental pathways in autism spectrum disorder: a review of signaling mechanisms and crosstalk. J. Neurodev. Disord. 11, 10 (2019).

31. Goodman, A. B. Three independent lines of evidence suggest retinoids as causal to schizophrenia. Proc. Natl Acad. Sci. USA 95, 7240-7244 (1998).

32. Reay, W. R. et al. Polygenic disruption of retinoid signalling in schizophrenia and a severe cognitive deficit subtype. Mol. Psychiatry 25, 719-731 (2018).

33. Rilling, J. K. Comparative primate neurobiology and the evolution of brain language systems. Curr. Opin. Neurobiol. 28, 10-14 (2014)

34. Chiang, M. Y. et al. An essential role for retinoid receptors RAR $\beta$ and RXRY in long-term potentiation and depression. Neuron 21, 1353-1361 (1998)

35. Krezel, W. et al. Impaired locomotion and dopamine signaling in retinoid receptor mutant mice. Science 279, 863-867 (1998)

36. Rossant, J. et al. Expression of a retinoic acid response element-hsplacZ transgene defines specific domains of transcriptional activity during mouse embryogenesis. Genes Dev. 5, 1333-1344 (1991).

37. Wilde, J. J. et al. Diencephalic size is restricted by a novel interplay between GCN5 acetyltransferase activity and retinoic acid signaling. J. Neurosci. 37, 2565-2579 (2017).

38. Yashiro, K. et al. Regulation of retinoic acid distribution is required for proximodistal patterning and outgrowth of the developing mouse limb. Dev. Cell 6, 411-422 (2004).

Publisher's note Springer Nature remains neutral with regard to jurisdictional claims in published maps and institutional affiliations.

(c) The Author(s), under exclusive licence to Springer Nature Limited 2021 


\section{Methods}

\section{Data reporting}

No statistical methods were used to predetermine sample size. Data collection was performed by independent investigators. Prior to data analysis, all experiments were randomized and analysed by independent blinded observers.

\section{Analysis of human and macaque transcriptomic data}

Developing human and macaque brain RNA-seq data (counts file) with the metadata information were obtained from BrainSpan (https:// brainspan.org/) and PsychENCODE (http://development.psychencode. org/; http://evolution.psychencode.org/) projects ${ }^{17,19}$. The timelines of human and macaque development and associated periods were reported in a previous study ${ }^{39}$.

For human mid-fetal periods 4-6, a total of 73 mRNA samples corresponding to 11 prospective neocortical areas, comprising the pial surface, marginal zone, cortical plate (layers 2-6) and adjacent subplate zone, from windows 3 and 4 (PCW 16-22) were considered for analyses (Extended Data Fig. 1). The human neocortical areas under study are the orbital (oPFC or OFC), dorsolateral (dIPFC or DFC), ventrolateral (vIPFC or VFC), medial (mPFC or MFC) prefrontal cortex and primary motor cortex (M1C) from the frontal lobe; primary somatosensory cortex (S1C) and posterior inferior parietal cortex (IPC) from the parietal lobe; primary auditory cortex (A1C), posterior superior temporal cortex (STC) and inferior temporal cortex (ITC) from the temporal lobe; and primary visual cortex (V1C) from the occipital lobe. A TMM normalization procedure was applied (function normalizeCounts from tweeDEseq package in $\mathrm{R}$ ) to the expression of 15,724 protein-coding genes that show sufficiently large counts (determined with function filterByExpr from edgeR package in R). To identify genes that are upregulated in a given brain lobe, we first applied RNentropy ${ }^{40}$, available as a package in $\mathrm{R}$, to determine which genes are differentially expressed among the 11 neocortical areas. Then, we considered a gene to be specifically overexpressed in a given lobe if (1) there is at least one area in this lobe where the gene is significantly upregulated; (2) the gene is not upregulated in any area of the other lobes; and (3) the gene is under-expressed in at least $30 \%$ of the areas from the remaining lobes. Similarly, we identified genes that are specifically upregulated in the PFC compared to the M1C, or vice versa, by first running RNentropy pairwise comparisons between M1C and each of the prefrontal areas independently. Then, a gene was considered to be upregulated in PFC if (1) it is upregulated in a prefrontal area in at least one of the comparisons; (2) it is not upregulated in M1C in any of the comparisons; and (3) it is under-expressed in $\mathrm{M} 1 \mathrm{C}$ in at least three of the comparisons. A gene was considered to be upregulated in M1C if (1) it is overexpressed in MOp in at least three of the comparisons; (2) it is not upregulated in any PFC area; and (3) it is under-expressed in a prefrontal area in at least one of the comparisons. Principal component analyses were performed using the prcomp function in $\mathrm{R}$ by centring the $\log _{2}$-transformed expression data of the selected genes. Significant GO terms were obtained with the goana function from the limma package in $\mathrm{R}$, reporting the ones with at least 10 genes in the background and at least 5 in the dataset. Frontal lobe, PFC and $\mathrm{M} 1 \mathrm{C}$ upregulated genes were characterized for association with RA signalling, autism spectrum disorder (ASD) and schizophrenia (SCZ) using the following criteria. Association with RA signalling was defined both by dysregulation in the PD 0 Rarb and $R x r g$ double-knockout frontal cortex RNA-sequencing dataset and by literature review identifying association with RA signalling. Association with ASD was based on https://gene.sfari.org/database/human-gene/. Association with SCZ was based on a total score of 3 or higher on http://www.szdb.org/ gene_rank.php. Information is provided in Supplementary Table 3.

Predicted ages for macaque samples were calculated via the TranscriptomeAge algorithm described previously ${ }^{19}$. To perform statistical comparisons, samples from various developmental periods were grouped (periods 4-6, 7-10 and 11-14) and a two-tailed Student's $t$-test was used to compare gene expression levels between brain regions and species.

\section{Animals}

All studies using mice (Mus musculus) and rhesus macaques (Macaca mulatta) were performed in accordance with protocols approved by Yale University's Institutional Animal Care and Use Committee and National Institutes of Health (NIH) guidelines. The animals were housed, and timed-pregnant prenatal and post-natal mouse and monkey brains were obtained in-house at the Yale Animal Resource Center.

Mice were reared in group housing less than five mice per cage at $25^{\circ} \mathrm{C}$ and $56 \%$ humidity in a 12-h light:12-h dark cycle and provided food and water ad libitum with veterinary care provided by Yale Animal Resource Center. Both sexes were used and randomly assigned for all experiments. Animals were maintained on the C57BL/6J background. The day on which a vaginal plug was observed was designated as PCD 0.5 in mice. For timed pregnancies in monkeys, females were housed with males for three days and the middle day was designated after observation of a vaginal plug and subsequent pregnancy as PCD 1. By this method, the estimated age of a monkey fetus has a maximal variation of \pm 1 day in its 165 -day gestation. All monkeys tested negative for herpes B virus and tuberculosis. RARE-lacZ(Tg(RARE-Hspa1b/lacZ)12Jrt) mice and timed-pregnant CD-1 mice for in utero electroporation were purchased from Jackson Laboratory and Charles River Laboratories, respectively. Sex of mouse samples in this study was not characterized.

\section{Post-mortem human and macaque tissue}

This study was conducted using post-mortem human brain specimens or RNA-seq data generated previously ${ }^{17}$ from tissue collections at the Department of Neuroscience at Yale School of Medicine, the Brain and Tissue Bank for Developmental Disorders at the University of Maryland, the Clinical Brain Disorders Branch of the National Institute of Mental Health, the Human Fetal Tissue Repository at the Albert Einstein College of Medicine, the Birth Defects Research Laboratory at the University of Washington (R24HD000836), Advanced Bioscience Resources and the Joint MRC-Wellcome Trust Human Developmental Biology Resource (www.hdbr.org; MR/R006237/1). Tissue was collected after obtaining parental or next of kin consent and with approval by the institutional review boards at each institution from which tissue specimens were obtained, the Yale University and the NIH. Donated deidentified tissue was handled in accordance with ethical guidelines and regulations for the research use of human brain tissue set forth by the NIH (https://oir.nih.gov/sites/default/files/uploads/ sourcebook/documents/ethical_conduct/guidelines-biospecimen.pdf) andtheWMADeclaration ofHelsinki(https://www.wma.net/policies-post/ wma-declaration-of-helsinki-ethical-principles-for-medical-researchinvolving-human-subjects/). All available non-identifying information was recorded for each specimen. No obvious signs of neuropathological alterations were observed in any of the human or macaque specimens analysed in this study. The post-mortem interval was defined as hours between time of death and time when tissue samples were fresh-frozen or started to undergo fixation process. We strived to keep warm ischaemia to a minimum and most of the post-mortem interval was composed of cold ischaemic time.

\section{Generation of Rarb, Rxrg and Cyp26b1 knockout mice using the CRISPR-Cas9 gene-editing technique}

The overall strategy for the generation of Rarb and Rxrg KO mice follows a previously described protocol using the CRISPR-Cas9 genome editing technique $\mathrm{e}^{41}$. For the construction of the templates of guidance RNAs, two sets of top and bottom strand oligomers (see Supplementary Table 4) directing the double-strand break at targeting sites were annealed and ligated into the BbsI site of a pX330-U6-Chimeric BB-CBh-hSpCas9 vector ${ }^{42}$, which was purchased from Addgene 
(plasmid 42230). After amplification of the insert with T7-tagged primers (Supplementary Table 5), guidance RNAs were synthesized by T7 RNA polymerase. The coding region of Cas 9 was PCR-amplified using pX330-U6-Chimeric_BB-CBh-hSpCas9 as a template and inserted into the pSP64 Poly(A) vector (Promega, P1241). Vectors were digested and linearized with EcoRI. Cas 9 mRNA was synthesized by SP6 RNA polymerase. Guidance RNAs and Cas9 mRNA was purified by the MEGAclear Transcription Clean-Up Kit (Ambion, AM1908). Cas9 mRNA and two guidance RNAs were mixed at a concentration of $10 \mathrm{ng}^{-1} \mathrm{l}^{-1}$ or $100 \mathrm{ng} \mathrm{\mu l}^{-1}$ in the microinjection buffer ( $5 \mathrm{mM}$ Tris- $\mathrm{HCl} \mathrm{pH} \mathrm{7.5;0.1} \mathrm{M} \mathrm{EDTA)} \mathrm{and}$ injected into the pronuclei of fertilized eggs from the B6SJLF1/J mouse strain purchased from The Jackson Laboratory. The fertilized eggs were then transferred to the uterus of females of the CD-1 mouse strain, purchased from Charles River Laboratories. The first generation $\left(\mathrm{F}_{0}\right)$ mice with recombined alleles were identified by PCR with two primer sets designed outside and inside of the targeted area (Supplementary Table 5, Extended Data Fig. 9d), confirmed by genome DNA sequencing. The germline transmission in the $F_{1}$ generation was confirmed by the same sets of PCR primers. For generation of Rxrg KO mice, a pair of guidance RNAs flanking whole exon 3 and 4 were designed to delete a large part of the DNA-binding domain ${ }^{43}$ (Extended Data Fig. 8d). For generation of Rarb KO mice, a pair of guidance RNAs was designed to delete the whole of exon 9 and a part of exon 10 (Extended Data Fig. 8d). As a result, $\alpha$-helical sheets of $\mathrm{H} 4$ to $\mathrm{H} 8$ in the ligand-binding domain were deleted, and a frameshift occurred in the rest of the $\mathrm{C}$-terminal region, which results in total abolition of receptor activity ${ }^{43}$. For generation of Cyp26b1 KO mice, a pair of guidance RNAs was designed to delete the whole of exon 3 and 6, as described previously, to abolish enzymatic activity ${ }^{44}$ (Extended Data Fig. 18a). All primer sequences are listed in Supplementary Table 5.

\section{Human primary neocortical cultures and differentiation}

Fresh tissues from prenatal human brain specimens (PCW 8-HSB\#765, sex not determined; PCW 20-HSB\#781, female; PCW 23-HSB\#784, male) were maintained in ice-cold Hibernate-E (Thermo Fisher Scientific, A1247601) and processed within 12 to $18 \mathrm{~h}$ post-mortem interval. Primary cortical neural stem cells from PCW 8 cortical tissue were isolated from the dissected neocortical proliferative zones (that is, the ventricular zone and subventricular zone). Primary cortical neural progenitors and neurons from PCW 20 or PCW 23 cortical tissue were isolated from dissected neocortical walls, including cortical plate zones. In brief, the tissue was mechanically separated into small pieces, incubated with $2 \mathrm{mg} \mathrm{ml}^{-1}$ papain (Brainbits, PAP) for $20 \mathrm{~min}$, and gently triturated to a single-cell suspension with $0.1 \mathrm{mg} \mathrm{ml}^{-1}$ DNase I (STEMCELL Technologies, 07900) in HBSS (Thermo Fisher Scientific, 88284). Cortical neural stem cell expansion and differentiation were performed as previously described $^{45}$. Cells were plated onto poly-L-ornithine/laminin-coated wells at a density of $2 \times 10^{5}$ cells in a 24 -well plate (IBIDI) and cultured with DMEM/F12 supplemented with $1 \times$ N2 (Thermo Fisher Scientific, 17502048), 1× B27 (Thermo Fisher Scientific, 17504044), 10 ng FGF2 (R\&D Systems, 3718-FB) and 1\% penicillin-streptomycin (Thermo Fisher Scientific, 15140122). The expanded cortical stem cells were replated onto poly-L-ornithine/laminin-coated wells at a density of $1 \times 10^{5}$ cells in a 24-well plate, and differentiation to neurons was induced two days after plating by FGF 2 withdrawal. The isolated cortical neural progenitors and neurons were plated onto poly-L-ornithine/laminin-coated wells at a density of $2 \times 10^{5}$ cells in a 24 -well plate (Ibidi) and cultured without FGF2. On day 4 after the FGF2 withdrawal of cortical neural stem cell culture and day 2 of cortical progenitor and neuron culture, the medium was replaced with neurobasal medium (Thermo Fisher Scientific, 21103049) containing $1 \times$ N2 (Thermo Fisher Scientific, 17502048), $1 \times$ B27 (Thermo Fisher Scientific, 17504044), $10 \mathrm{ng} \mathrm{ml}^{-1}$ BDNF (Abcam, 9794), $10 \mathrm{ng} \mathrm{ml}^{-1}$ GDNF (R\&D Systems, 212-GD) and 1\% penicillin-streptomycin (Thermo Fisher Scientific, 15140148). After neural differentiation and further maturation (Extended Data Fig. 15a), cortical neurons were exposed to varying doses of all-trans retinoic acid (Sigma-Aldrich, R2625-50MG) or the pan-retinoic acid receptor antagonist AGN193109 (Tocris, 5758) for another 14 days. See Extended Data Fig. 15a for a summary.

\section{Human and chimpanzee cerebral organoid culture}

All human and chimpanzee cell lines were authenticated by morphology or genotyping, and tested negative for mycoplasma contamination, checked monthly using the MycoAlert Mycoplasma Detection Kit (Lonza). For maintenance of human (cell line: HSB311 \#36 (refs. ${ }^{46,47}$ )) and chimpanzee (cell line:3651D $\left(\right.$ ref. $\left.^{48}\right)$ ) cell lines, induced pluripotent stem (iPS) cells were dissociated to single cells with Accutase (Thermo Fisher Scientific, 00-4555-56) and plated at a density of $1 \times 10^{5}$ cells per $\mathrm{cm}^{2}$ in Matrigel (BD)-coated 6-well plates (Falcon) with mTeSR1(STEMCELL Technologies, 85850) containing $5 \mu \mathrm{M}$ Y27632, ROCK inhibitor (Sigma-Aldrich, Cat.SCM075). ROCK inhibitor was removed at $24 \mathrm{~h}$ after plating, and cells were cultured for another four days before the next passage. Cerebral organoids were generated by directed differentiation protocol as previously described ${ }^{45-47}$. Human and chimpanzee iPS cells were dissociated into single cells using Accutase (Thermo Fisher Scientific, 00-4555-56). Neural induction was directed by dual SMAD and WNT inhibition using neural induction medium supplemented with 100 nM LDN193189 (STEMCELL Technologies, 72147), 10 M SB431542 (Selleck Chemicals, S1067) and $2 \mu$ M XAV939 (Sigma-Aldrich, X3004$5 \mathrm{MG})$. The dissociated cells were reconstituted with the neural induction medium and plated at 10,000 cells per well in a 96-well v-bottom ultra-low-attachment plate (Sumitomo Bakelite). To increase the cell survival and aggregate formation, $10 \mu \mathrm{M}$ Y-27632 (Sigma-Aldrich, SCM075) was added for the first day. After 10 days of stationary culture, organoids were transferred to a 6-well ultra-low-attachment plate (Millipore Sigma) and kept on an orbital shaker rotating at a speed of 90 rpm to enhance the nutrient and gas exchanges. From day 18, organoids were cultured with neural differentiation medium supplemented with $1 \times$ CD lipid concentrate (Thermo Fisher Scientific, 11905031), $5 \mathrm{\mu g} \mathrm{ml}^{-1}$ heparin (STEMCELL Technologies, 07980), $20 \mathrm{ng} \mathrm{ml}^{-1}$ BDNF (Abcam, 9794), $20 \mathrm{ng} \mathrm{ml}^{-1}$ GDNF (R\&D Systems, 212-GD), $200 \mu \mathrm{M} \mathrm{cAMP}$ (Sigma-Aldrich, 20-198) and $200 \mu \mathrm{M}$ ascorbic acid (Sigma-Aldrich, A92902) for further neuronal maturation (Extended Data Fig. 10a). On day 133, an RA-soaked bead was attached to each cerebral organoid by embedding with and solidifying the growth-factor-reduced Matrigel (Corning, 354230) and further cultured for $48 \mathrm{~h}$ before collection.

\section{Preparation of RA-soaked beads}

AG1-X2 Resin (150 or $200 \mu \mathrm{m}$ in diameter, Bio Rad, 140-1231) were added with $1 \mathrm{M}$ formic acid for $1 \mathrm{~h}$ and washed with distilled $\mathrm{H}_{2} \mathrm{O}(5 \times 5 \mathrm{~min})$. Beads were completely dried in a $37^{\circ} \mathrm{C}$ incubator overnight. Dried beads were soaked in either $1 \mathrm{mg} \mathrm{ml}^{-1}$ or $5 \mathrm{mg} \mathrm{ml}^{-1}$ of all-trans retinoic acid (Sigma-Aldrich, R2625-50MG) dissolved in DMSO (Sigma-Aldrich, D8418) for $1 \mathrm{~h}$ and then washed twice in DMEM (Gibco) before being placed onto human or chimpanzee organoids.

\section{In situ hybridization}

Whole-mount and section in situ hybridization was performed as described previously ${ }^{49}$. Antisense digoxigenin (DIG)-labelled RNA probes were synthesized using DIG RNA Labeling Mix (Roche, 11277073910). Human and mouse ALDH1A1 (Clone ID 2988388 and 6477503, respectively), ALDH1A3 (Clone ID 6208628 and 6515355, respectively), $R X R G$ (Clone ID 4635470 and 5707723, respectively), $R A R B$ (Clone ID 30341884 and 30608242, respectively) and mouse Rorb (Clone ID 5358124), Cbln2 (Clone ID 6412317), Cyp26b1 (Clone ID 6400154) cDNAs were purchased from GE Healthcare for template preparation. Mouse Meis2 DNA was a gift from J. Rubenstein. For macaque in situ hybridization, human probes were used because of high similarity between human and macaque transcripts (97.7\% identity in $A L D H 1 A 1$; $95.4 \%$ identity in $A L D H 1 A 3 ; 97.7 \%$ in $R X R G$; and $98.8 \%$ in 
$R A R B)$. Sections were obtained from PCW 21, 22 human brains and PCD 110,140 macaque brains. In situ hybridization were repeated using these two sets. Images were taken using an Aperio CS2 HR Scanner (Leica) and processed by Aperio ImageScope (v.12.4.3.5008, Leica). In Fig. 5a, Cbln2, Cyp26b1 and Rorb expression data were merged by converting colour whole-mount in situ hybridization into black-and-white images, then merged as separate RGB channels. Images were aligned manually by N.S. In Fig. 5f, Extended Data Fig. 19a, the cortical plate was divided into five equal bins and Rorb intensity in each bin was quantified using ImageJ (v.2.0.0-rc-69/1.52p).

\section{ELISA}

Eleven neocortical areas were dissected from four fresh frozen post-mortem human mid-fetal brains (PCW 16, 18,18 and 19) and four fresh frozen macaque brains (all four PCD110) as described previously ${ }^{19}$. Twelve neocortical areas were dissected from three fresh mouse brains at PD 1, based on Paxinos ${ }^{50}$ and the Allen Mouse Brain Atlas ${ }^{51}$ (https:// mouse.brain-map.org/static/atlas). Each human and macaque brain sample was further microdissected into three pieces and weighed. Each piece was independently homogenized using a Dounce homogenizer in three to four volumes of homogenizing buffer (isopropanol:ethanol $2: 1 ; 1 \mathrm{mg} \mathrm{ml}^{-1}$ butylated hydroxytoluene), followed by centrifugation at $10,000 \mathrm{rpm}$ for $10 \mathrm{~min}$ at $4^{\circ} \mathrm{C}$. Supernatant was used for the determination of both protein concentration by Pierce BCA Protein Assay Kit (Thermo Fisher Scientific, 23227), and RA concentrations using an ELISA colorimetric detection kit according to the manufacturer's instructions (MyBioSource, MBS705877). This kit could not distinguish all-trans retinol, all-trans retinal or all-trans retinoic acid). Thus, the concentration of RA was the concentration of all forms.

\section{Quantitative PCR with reverse transcription}

Total RNA was isolated from freshly microdissected cortices after removal of the olfactory bulb and striatum (Extended Data Fig. 9a, b) using Trizol (Thermo Fisher Scientific, 15596026). cDNAs were prepared using SuperScript II (Thermo Fisher Scientific, 18064022) from three independent wild-type cerebral hemispheres. Quantitative PCR with reverse transcription was performed as described previously ${ }^{52}$ using the 7000HT Sequence Detection System (Applied Biosystems). At least three replicates per transcript were used for every reaction. The copy number of transcripts was normalized against the housekeeping TATA-binding protein (TBP) transcript level. For Rxra, Rxrb, Rxrg, Rara, Rarb, Rarc and $T b p$ primer sets, the correlation $\left(R_{2}\right)$ was higher than 0.98 , and the slope was -3.1 to -3.6 in each standard curve. Primers to detect the expression of the genes above were designed in a single exon. Primer sequences are listed in Supplementary Table 5.

\section{$\boldsymbol{\beta}$-Galactosidase histochemical staining}

Brains were dissected from PD 0 RARE-lacZ mouse pups and drop-fixed in $4 \%$ paraformaldehyde for $2 \mathrm{~h}$ at $4{ }^{\circ} \mathrm{C}$, followed by embedding in OCT compound (Thermo Fisher Scientific, 23-730-572). Brains were sectioned at $20 \mu \mathrm{m}$ by cryostat (Leica CM3050S) after they were frozen. $\beta$-Galactosidase staining followed the protocol described previously ${ }^{53}$. We used Red-gal (Sigma-Aldrich, RES1364C-A102X) for the chromogenic reaction. The intensity of $\beta$-galactosidase staining was quantified using ImageJ (v.2.0.0-rc-69/1.52p).

\section{Nissl staining}

Post-mortem brains were immersion fixed in $4 \%$ paraformaldehyde overnight at $4{ }^{\circ} \mathrm{C}$, cryoprotected in $30 \%$ sucrose, embedded in OCT compound (Thermo Fisher Scientific, 23-730-572) and snap-frozen in dry ice chilled isopentane (2-methylbutane). Brains were sectioned at 15-20 $\mu \mathrm{m}$ by cryostat (Leica, CM3050S) after they were frozen. After PBS wash, sections were dehydrated using an increasing concentration of ethanol, followed by Cresyl violet, wash, and a second ethanol dehydration. Sections were cover-slipped with Permount (Thermo Fisher Scientific, 15820100).

\section{Immunohistochemistry}

Human (PCW 12, PCW 16, PCW 19, PCW 20, PCW 21, PCW 22, PCW 24 , newborn, and adult (42,47 and 79 years old; post-mortem interval (PMI) 5-15 h)), macaque (PCD 40, PCD 76, PCD 114, PCD 140, adult (4.5, 8 and 11 years old; PMI under $1 \mathrm{~h}$ )) and mouse (PD 0 and adult (4 months)) brain tissue samples, the cultured human cortical neurons and the cerebral organoids were fixed in $4 \%$ paraformaldehyde at $4{ }^{\circ} \mathrm{C}$ and cryoprotected in graded concentrations of sucrose $(15 \%, 20 \%, 30 \%)$. Brain tissues and cerebral organoids were embedded in OCT compound (Thermo Fisher Scientific, 23-730-572) and snap-frozen in dry ice chilled isopentane (2-methylbutane). Embedded samples were sectioned at 15-20 $\mu \mathrm{m}$ for mouse brain and cerebral organoids, and $50 \mu \mathrm{m}$ for macaque and human brain by cryostat (Leica, CM3050S). Tissue sections were mounted and dried overnight.

For the immunohistochemistry of mouse brain in Extended Data Fig. 16d-f, sections were treated with or without R-Buffer AG pH 6.0 (Electron Microscopy Sciences, 62707-10) for antigen retrieval, followed by washing in PBS $(3 \times 5 \mathrm{~min})$ and incubation in blocking buffer (5\% (v/v) normal donkey serum (Jackson ImmunoResearch Laboratories, 017-000-121), 1\% (w/v) bovine serum albumin, (Sigma-Aldrich, A9647-100G), and 0.3\% (v/v) Triton X-100 in PBS) for $1 \mathrm{~h}$ at room temperature. Sections were incubated for $24-48 \mathrm{~h}$ at $4{ }^{\circ} \mathrm{C}$ with diluted primary antibodies in blocking buffer. Sections were then washed in PBS $(3 \times 5 \mathrm{~min})$ incubated with fluorescent secondary antibodies for $2 \mathrm{~h}$ at room temperature. After washing in PBS $(3 \times 5 \mathrm{~min})$, Tissue was cover-slipped with Vectashield (Vector Laboratories, H-1000). For the immunohistochemistry of human, macaque and mouse brain sections in Extended Data Figs. 4d, e, 7b, antigen retrieval was performed using R-Buffer A pH 6.0 (Electron Microscopy Sciences, 62706-10) for post-natal tissue or R-Buffer AG pH 6.0 (Electron Microscopy Sciences, 62707-10) for prenatal tissue. Sections were incubated in $1 \%$ hydrogen peroxide/PBS to quench endogenous peroxidase activity. Sections were washed in PBS $(3 \times 10 \mathrm{~min})$ and incubated in blocking solution containing $5 \%(\mathrm{v} / \mathrm{v})$ normal donkey serum (Jackson ImmunoResearch Laboratories, 017-000-121), 1\% (w/v) bovine serum albumin (Sigma-Aldrich, A9647-100G), and 0.3\% (v/v) Triton X-100 in PBS for $1 \mathrm{~h}$ at room temperature. Primary antibodies were diluted in blocking solution and incubated with tissues sections overnight at $4{ }^{\circ} \mathrm{C}$. Sections were washed with PBS $(3 \times 10 \mathrm{~min})$ before being incubated with the appropriate biotinylated or fluorescent secondary antibodies (Jackson ImmunoResearch Labs) for $1.5 \mathrm{~h}$ at room temperature. All secondary antibodies were raised in donkey and diluted at 1:250 in blocking solution. Sections were subsequently washed in PBS and incubated with avidin-biotin-peroxidase complex (Vectastain ABC Elite kit; Vector Laboratories) for $1 \mathrm{~h}$ at room temperature. Finally, sections were washed in PBS $(3 \times 15 \mathrm{~min})$ and signals were developed using a DAB Peroxidase (HRP) Substrate Kit (Vector Laboratories, NC9276270) according to the manufacturer's protocol. Following washes in PBS, sections were dried, dehydrated and cover-slipped with Permount (Thermo Fisher Scientific, 15820100). For immunofluorescence, sections were washed in PBS with $0.3 \%(\mathrm{v} / \mathrm{v})$ Triton X-100 and treated with the Autofluorescence Eliminator Reagent (Millipore, 2160) according to the manufacturer's instructions, and cover-slipped with Vectashield (Vector Laboratories, H-1000). For the immunocytochemistry shown in Extended Data Fig. 15, the fixed primary cortical neurons were washed in PBS $(3 \times 15 \mathrm{~min})$ and incubated with $0.3 \%(\mathrm{v} / \mathrm{v})$ Triton X-100 and $10 \%(\mathrm{v} / \mathrm{v})$ donkey serum (Jackson ImmunoResearch Laboratories, 017-000-121) in PBS for 40 min. For the immunohistochemistry shown in Extended Data Fig. 11a, b, the cerebral organoid sections were washed in PBS $(3 \times 15 \mathrm{~min})$ and incubated with $0.5 \%(\mathrm{v} / \mathrm{v})$ Triton X-100 and $10 \%(\mathrm{v} / \mathrm{v})$ donkey serum (Jackson ImmunoResearch Laboratories, 017-000-121) in PBS for 2 h. Primary cortical neurons and the organoid sections were incubated with primary antibodies in $10 \%(\mathrm{v} / \mathrm{v})$ donkey serum at $4{ }^{\circ} \mathrm{C}$ overnight. Samples were then washed in PBS $(3 \times 5 \mathrm{~min})$, and incubated with fluorescent 
secondary antibodies for $2 \mathrm{~h}$ at room temperature in $10 \%(\mathrm{v} / \mathrm{v})$ donkey serum. All sections and tissues processed for immunofluorescence were cover-slipped with Vectashield (Vector Laboratories, H-1000), after washing in PBS $(3 \times 5 \mathrm{~min})$.

The sources of primary antibodies were anti-ALDH1A1 (1:500, Abcam, 52492), anti-BCL11B/CTIP2 (1:500, Abcam, ab18465), anti-cleaved caspase3 (1:500; Cell Signaling, 9611), anti-CUX1 (1:500, Santa Cruz Biotechnology, sc-13024), anti-GFAP (1:2,000, Sigma-Aldrich, G3893), anti-NR4A2 (1:100, R\&D systems, AF2156), anti-GAD1 (1:50, R\&D systems, AF2086), anti-NRGN (1:50, R\&D systems, AF7947), anti-PAX6 (1:200; R\&D Systems, AF8150), anti-POU3F2/BRN2 (1:500, Sigma-Aldrich, SAB2702086), anti-DLG4/PSD95 (1:500; Invitrogen, 51-6900), anti-SYP (1:2,000, Sigma-Aldrich, SAB4200544), anti-L1CAM (1:500; Millipore-Sigma, ABT143), anti-RORB (1:500, Novus Biologicals, NBP1-82532), anti-SATB2 (1:200, Abcam, ab92446), anti-SOX2 (1:200, R\&D Systems MAB2018), anti-TBR1 (1:200, Abcam, ab31940), anti-TH (1:1,000, Immunostar, 22941), and anti-TUBB3 (1:400; TuJ1 clone, Abcam,18207). Secondary antibodies: Alexa Fluor 488-, 594-, or 647-conjugated AffiniPure Donkey anti-IgG (1:200; Jackson ImmunoResearch). For all microscopic analysis, images were acquired using an Aperio CS2 HR Scanner (Leica), ApoTome. 2 microscope (Zeiss), LSM510 META (Zeiss), LSM800 or LSB880 confocal microscope (Zeiss) and assembled in Aperio ImageScope 12.4.3.5008 (Leica), Zeiss ZEN2009, ImageJ (v.2.0.0-rc-69/1.52p), Adobe Photoshop (v.12.0x64), and Adobe Illustrator (v.23.1.1).

\section{Western blotting}

Five prospective neocortical areas (mPFC, dIPFC, vIPFC, oPFC and M1C) were dissected from fresh frozen brains of human and macaque, and three neocortical areas (mPFC, MOs/p, and OFC) from fresh mouse brains. Dissected tissues were lysed by sonication in RIPA buffer with protease inhibitors (Sigma-Aldrich, 11836153001). Following centrifugation at $10,000 \mathrm{~g}$, supernatant protein concentrations were quantified using the Pierce BCA Protein Assay Kit (Thermo Fisher Scientific, 23227). Supernatants were mixed with $4 \times$ Laemmli Sample Buffer (Bio Rad, 1610747), boiled, and electrophoresed on NuPage Bis-Tris gels (Thermo Fisher Scientific), followed by protein transfer to PVDF membranes (Bio Rad, 1620174). Blotted membranes were incubated in blocking buffer (TBS/5\% milk) for $1 \mathrm{~h}$ and then transferred to blocking buffer with primary antibodies diluted at 1:1,000 at $4{ }^{\circ} \mathrm{C}$ overnight. Membranes were then washed in TBS/0.1\% Tween $20(3 \times 5 \mathrm{~min})$, and incubated with secondary antibodies conjugated with HRP. Secondary antibodies were diluted at 1:1,000. After washing with TBS/0.1\% Tween 20 ( $3 \times 5 \mathrm{~min})$, protein bands were visualized using SuperSignal West Pico Chemiluminescent Substrate (Thermo Fisher Scientific, 34580). The sources of primary antibodies were anti-ALDH1A3 (1:2,000, Novus Biologicals, NBP2-15339) and anti-GAPDH (1:5,000, Abcam, 9485). Of note, two bands were identified using anti-ALDH1A3 antibody, a 56-kDa band likely to be ALDH1A3 and a $54 \mathrm{kDa}$ band likely to be ALDH1A1.

\section{Droplet digital PCR for quantification of gene expression}

An aliquot of the total RNA that was extracted from meninges (human: PCW 19, 19 and 20; macaque: PCD 80, 80 and 110) or primary neural cultures were used for secondary validation through droplet digital PCR (ddPCR) analysis. Two hundred nanograms of total RNA was used for cDNA synthesis using SuperScript III First-strand synthesis Supermix (Thermo Fisher Scientific, 18018400) and subsequently diluted with nuclease-free water. ddPCR was carried out using the Bio-Rad QX100 system. After each PCR reaction mixture consisting of ddPCR master mix and custom primers/probe set was partitioned into 15,000-20,000 droplets, parallel PCR amplification was carried out. End-point PCR signals were quantified and Poisson statistics were applied to yield the target copy number quantification of the sample. A two-colour $P C R$ reaction was used for the normalization of gene expression by the housekeeping gene TBP. All pre-designed assays used in validation can be found in Supplementary Table 5. For each region, the gene expression was compared between species by one-way ANOVA followed by Dunnett's multi comparison test.

\section{Quantification of post-synaptic and presynaptic puncta marked by immunostaining}

For each region of both wild-type and dKO mice, using the 488-nm or 594-nm channels to detect synaptophysin (SYP) immunolabelling, DLG4 (PSD95) immunolabelling and DAPI nuclear counterstain, seven serial optical sections at $0.8-\mu \mathrm{m}$ intervals over a total depth of $5 \mu \mathrm{m}$ were imaged and the $2 \mathrm{nd}, 4$ th and 6 th images were eliminated from further analysis to avoid overlap in counting ${ }^{54}$. The area of each image is $0.079 \mathrm{~mm}^{2}$. The number of SYP- and PSD95-immunolabelled puncta on each image was counted using ImageJ (v.2.0.0-rc-69/1.52p) using the automated Analyze Particles function using a threshold of 985 to 4,095, determined based on multiple wild-type and dKO images. At least two sections from each mouse were selected for counting, and at least three mice for each genotype were used. The total number and volume of SYP- and DLG4 (PSD95)-immunolabelled puncta and TuJ1 (anti-TUBB3 antibody) or MAP2 in cultured neurons were analysed with $20 z$-stack ( $0.49 \mu \mathrm{m}$ intervals) images per each 8 fields for each condition and the total number of DLG4 (PSD95)-immunolabelled puncta and TuJ1 immunolabelled neurons in cerebral organoids were analysed with 5 $z$-stack images using Volocity (v.6.3.1) and Spotfire (v.11.2.0) software.

\section{Retrograde neuronal tracing with adeno-associated viruses}

In brief, wild-type $(n=4)$ and Rarb;Rxrg dKO $(n=4)$ mice were anaesthetized by injecting ketamine/xylazine solution and head-fixed in the stereotactic frame. Mice were injected with burenorphine $30 \mathrm{~min}$ before the surgery. After lubricating the eyes and shaving the fur, an incision of less than $1 \mathrm{~mm}$ was made. A craniotomy was made with the round 0.5-mm drill bit at the desired co-ordinates (mPFC: $\mathrm{ML} \pm 0.35$, AP 1.5, DV 2.5 from bregma). Using a 0.5- $\mu$ l Hamilton neuros syringe, we injected $50 \mathrm{nl}$ of $A A V r g-C a g-G f p$ (Addgene, 37825-AAVrg) into the $\mathrm{mPFC}$. To prevent the virus from spreading along the injecting tract, the needle was held in place for at least $10 \mathrm{~min}$. After injections, the skin was sutured and mice were returned to the cage. After surgery, mice were injected with meloxicam for $48 \mathrm{~h}$. Three weeks later, the mice were euthanized and brains were collected. The brains were coronally sectioned on a vibratome to obtain $70-\mu \mathrm{m}$ thick sections. After staining the sections with anti-GFP antibody (1:500, Abcam, ab13970) and DAPI the sections were imaged with an LSM 800 microscope (ZEISS). The intensity and density of labelling was quantified on a scale of 0 to 3 with 0 being no labelling, 1 being weak or sparse cellular labelling (less than $10 \%$ ), 2 being strong and less than $50 \%$ of cell labelled, and 3 being dense labelling. This discrete approach of quantification was used owing to variability in injection site.

\section{Quantification of dendritic spines and arborization from viral tracing sections}

The contralateral mPFC to the injection site was used for this analysis; wild type $(n=4)$ and $R a r b ; R x r g d K O(n=4)$. For Sholl analysis, the images of entire neurons were acquired at $20 \times$ magnification. For spine counts, $z$-stack images across entire dendritic thickness with 29-33 images per stack were obtained. The $z$-stack images were opened in Reconstruct (v.1.1.0.0 $)^{55}$, which is publicly available at https://www.bu.edu/neural/ Reconstruct.html, and a new series was recreated that enabled us to move across different stacks across $z$-planes in the same image. Whole dendrite was subdivided into segments of $10 \mu \mathrm{m}$ and number of spines across whole thickness were traced for length and breadth of each spine. After tracing, the length and breadth of the spines their ratio was used to determine the spine subtype as described before ${ }^{56}$. After the analysis for each class of spine, standard deviation and $P$ values were calculated using two-way ANOVA with Sidak's multiple comparison method. For Sholl analysis, $z$-stack images were opened in ImageJ 
(v.2.0.0-rc-69/1.52p) and dendritic arbors were manually traced using the NeuronJ plugin ${ }^{57}$. Dendritic complexity was then quantified and plotted using the Sholl Analysis option.

\section{Plasmid construction}

For construction of expression vectors, full-length cDNAs (mouse Aldh1a3, Clone ID 6515355, purchased from GE Healthcare) were inserted into pCAGIG vector (pCAGIG was obtained from Addgene (plasmid 11159).

\section{In utero electroporation}

In utero electroporation was performed as previously described ${ }^{52}$. Plasmid DNA $\left(4 \mu \mathrm{g} \mathrm{ll}^{-1}\right)$ was injected into the lateral ventricle of embryonic mice at E13.5-E14.5 and transferred into the cells of the ventricular zone by electroporation (five 50-ms pulses of $40 \mathrm{~V}$ at 950 -ms intervals). Brains were dissected at PD 0 . Brains and tissue sections of electroporated mice were analysed for GFP expression after fixation with $4 \%$ paraformaldehyde for $12 \mathrm{~h}$ at $4{ }^{\circ} \mathrm{C}$.

\section{Mouse RNA-seq data generation and analysis}

Post-mortem mouse brains were dissected at PD 0 in ice-cold sterile PBS, fresh frozen, and stored at $-80^{\circ} \mathrm{C}$. Brains were incubated in RNAlater-Ice at $-20^{\circ} \mathrm{C}$ for $12-16 \mathrm{~h}$ prior to further dissection. mPFC, MOs, and OFC were microdissected based on Paxino ${ }^{50}$ and the Allen Mouse Brain Atlas $^{51}$ (https://mouse.brain-map.org/static/atlas) and RNA was isolated using RNeasy Plus Micro kit with additional on-column DNAase step (Qiagen, 74134). RNA quality and amount were quantified using the High Sensitivity RNA Screen Tape Assay (Agilent, 5067-5579), and the concentration was standardized to $10 \mathrm{ng} \mathrm{l}^{-1}$. The SMART-seq v4 Ultra Low Input Kit (Takara Bio, 634890) was used to create cDNA, and the concentration was quantified using Quant-iT PicoGreen dsDNA Assay Kit (Thermo Fisher Scientific, P11496). Nextera XT DNA library Preparation Kit (Illumina, FC-131-1024) was used to create cDNA libraries for sequencing. Libraries were normalized and sequenced at the Yale Center for Genomic Analysis (YGCA) using the NovaSeq with 100-bp paired end reads. Reads from each library were mapped against the mouse assembly GRCm38 using STAR v.2.6.0a (gtf and fasta files downloaded from Ensembl version 94; parameters:--readFilesIn $\$ \mathrm{j} 1$ \$j2--outSAMattributes All --outFilterMultimapNmax 1 --outSAMstrandField intronMotif --outFilterIntronMotifs RemoveNoncanonical --quantMode TranscriptomeSAM --outFilterMismatchNoverLmax 0.1--alignSJoverhangMin 8 --alignSJDBoverhangMin 1--outSAMunmapped Within --outFilterType BySJout). Counts were obtained using featureCounts v1.6.2 with -p parameter.

To compare the gene expression patterns of three wild-type versus three $R a r b$ and $R x r g$ dKO mice, a TMM procedure was applied (function normalizeCounts from tweeDEseq package in $\mathrm{R}$ ) to the expression of 15,085 protein-coding genes that show sufficiently large counts (determined with function filterByExpr from edgeR package in $\mathrm{R}$ ). We assessed differentially expressed genes in each brain region (mPFC, OFC and MOs) running RNentropy independently among wild-type and $\mathrm{dKO}$ mice per region. Genes overexpressed in a given condition are those that are both significantly upregulated in that condition and significantly downregulated in the opposite condition according to RNentropy. The same criterion was applied for the identification of downregulated genes. Genes with an inconsistent pattern of expression between regions were excluded. Principal component analyses were performed using the prcomp function in $\mathrm{R}$ by centring the $\log _{2}$-transformed expression data of the selected genes. Significant GO terms were obtained with the goana function from the limma package in $\mathrm{R}$ and plotted using the function GOBubble from the GOplot package in R. Fisher test enrichments calculated for RA-related genes (RA synthesis: Rdh10, Rdh5, Aldh1a1/Raldh1, Aldh1a2/Raldh2, Aldh1a3/Raldh3,Adh1,Adh5,Adh7; RA degradation:Cyp26a1,Cyp26b1, Cyp26c1; RA receptors: Rara, Rarb, Rarg, Rxra, Rxrb, Rxrg; RA binding:
$T t r, R l b p 1, R b p 1, R b p 2, R b p 3, R b p 4, F a b p 5)$, genes overexpressed in individual lobes of the mid-fetal human cortex based on Fig. 1, and neuropsychiatric-disease related genes (downloaded from ref. ${ }^{30}$.) in up- and downregulated genes. Genes associated with the GO terms: 'axon guidance', 'axon guidance receptor', 'axon development' and 'ephrin' were manually screened for anterior-to-posterior gradient using developingmouse.brain-map.org ${ }^{58}$, gensat.org ${ }^{59}$ and a previous report ${ }^{60}$. Mouse RNA-seq data were deposited into the NCBI Gene Expression Omnibus (GEO) database (accession number GSE142851).

\section{Diffusion-weighted MRI and tractography}

Five PD 5 post-mortem dKO homozygotes and five wild-type C57BL/6 mouse brains were drop-fixed in $4 \%$ paraformaldehyde solution in $0.1 \mathrm{M}$ PBS for $48 \mathrm{~h}$. They were subsequently transferred to $0.1 \mathrm{M}$ PBS and just before imaging to Fomblin (Sigma-Aldrich, 317926). The diffusion-weighted images were acquired on a Bruker BioSpin 9.4 T MRI (Bruker) using a standard 3D Stejskal-Tanner spin-echo sequence with 30 different angles of diffusion sensitization at a $b$ value of $1,000 \mathrm{~s} \mathrm{~mm}^{-2}$ and the following parameters: repetition time $=2,000 \mathrm{~ms}$; echo time $=$ $25.616 \mathrm{~ms}$; diffusion encoding duration $=4 \mathrm{~ms}$. The in-plane resolution was $0.11 \mathrm{~mm}$ and slice thickness was $0.22 \mathrm{~mm}$. Overall scanning time was around $24 \mathrm{~h}$.

\section{Image processing and tractography}

Cerebral cortical regions of interest (ROI) and thalamus were manually defined according to Paxinos ${ }^{50}$ and the Allen Mouse Brain Atlas ${ }^{51}$ (https:// mouse.brain-map.org/static/atlas) by D.A. and K.P. without prior knowledge of the experimental groups. Image preprocessing was done with Advanced Normalization Tools (ANTs, v.2.2.0.0.dev297-gf23cb). The reconstruction of axonal pathways was performed with MRtrix ${ }^{61}$ software (v.3.0.0-65-g91788533) using constrained spherical deconvolution $^{62}$ and probabilistic tracking (iFOD2) with a FOD amplitude cut-off of 0.1. The thalamus was used as a seeding point and each cortical ROI was used as a termination mask. To evaluate the integrity of the major white matter tracts between the groups, both internal capsules, anterior commissure and corpus callosum were manually delineated according to Paxinos ${ }^{50}$ and the Allen Mouse Brain Atlas ${ }^{51}$ (https://mouse. brain-map.org/static/atlas) by D.A. and K.P. without prior knowledge of the experimental groups. Values of the fractional anisotropy (FA), apparent diffusion coefficient (ADC), radial (RD) and axial (AD) diffusivity were calculated using underlying scalar maps derived by MRtrix3.

\section{Anterograde tracing of axons}

For anterograde tracing of axons between MPFC and thalamus, brains were collected at either PCD 18 or P21, and fixed overnight in $4 \%$ paraformaldehyde at $4{ }^{\circ} \mathrm{C}$. Brains were then hemidissected. A crystal of 1,1'-dioctadecyl-3,3,3',3'-tetramethylindocarbocyanine perchlorate (Dil, Sigma-Aldrich, 24364) was inserted into the mPFC, MD nucleus of the thalamus, or medial thalamus under the stereomicroscope. The size of the crystal is around $200 \mu \mathrm{m}$. Brains were then placed in $1 \%$ paraformaldehyde in PBS and left for 14 days at $37^{\circ} \mathrm{C}$. Following Dil diffusion, the brains were sectioned coronally on a vibrating microtome (Leica) at $80-\mu \mathrm{m}$ thickness and stained with DAPI. Sections were mounted onto glass and immediately sealed in VECTASHIELD Hardset Antifade Mounting Medium (Vector Laboratories, H-1400). Slides were analysed under a ApoTome. 2 microscope (Zeiss) and intensity was quantified using ImageJ (v.2.0.0-rc-69/1.52p).

\section{Processing, analysis and image visualization}

To allow robust visualization and analysis, images depicting Dil tracing or immunohistochemistry using antibody against PSD95 (DLG4) have been inverted and/or pseudo-coloured, as in Figs. 4, 5. In addition, background was removed for in situ hybridization experiments and images were pseudo-coloured and superimposed in Fig. 5, Extended Data Figs. 4, 7, 9, 10 using Adobe Photoshop. 


\section{Reporting summary}

Further information on research design is available in the Nature Research Reporting Summary linked to this paper.

\section{Data availability}

The mouse RNA-seq data are available at the NCBI GEO under the accession number GSE142851.

39. Kang, H. J. et al. Spatiotemporal transcriptome of the human brain. Nature $\mathbf{4 7 8}, 483-489$ (2011).

40. Zambelli, F. et al. RNentropy: an entropy-based tool for the detection of significant variation of gene expression across multiple RNA-Seq experiments. Nucleic Acids Res. 46, e46 (2018)

41. Wang, $\mathrm{H}$. et al. One-step generation of mice carrying mutations in multiple genes by CRISPR/cas-mediated genome engineering. Cell 153, 910-918 (2013).

42. Cong, L. et al. Multiplex genome engineering using CRISPR/Cas systems. Science $\mathbf{3 3 9}$, 819-823 (2013).

43. Renaud, J. P. et al. Crystal structure of the RAR-y ligand-binding domain bound to all-trans retinoic acid. Nature 378, 681-689 (1995)

44. MacLean, G. et al. Apoptotic extinction of germ cells in testes of Cyp26b1 knockout mice. Endocrinology 148, 4560-4567 (2007).

45. Micali, N. et al. Variation of human neural stem cells generating organizer states in vitro before committing to cortical excitatory or inhibitory neuronal fates. Cell Rep. 31, 107599 (2020).

46. Xiang, Y. et al. Fusion of regionally specified hPSC-derived organoids models human brain development and interneuron migration. Cell Stem Cell 21, 383-398 (2017)

47. Pollen, A. A. et al. Establishing cerebral organoids as models of human-specific brain evolution. Cell 176, 743-756 (2019).

48. Gallego Romero, I. et al. A panel of induced pluripotent stem cells from chimpanzees: a resource for comparative functional genomics. Elife 4, e07103 (2015).

49. Wilkinson, D. G. \& Nieto, M. A. Detection of messenger RNA by in situ hybridization to tissue sections and whole mounts. Methods Enzymol. 225, 361-373 (1993)

50. Paxinos, G. Atlas of the Developing Mouse Brain: at E17.5, PO, and P6 (Academic, 2007)

51. Dong, H. W. The Allen Reference Atlas: a Digital Color Brain Atlas of the C57BL/6J Male Mouse (John Wiley \& Sons, 2008).

52. Shibata, M. et al. MicroRNA-9 modulates Cajal-Retzius cell differentiation by suppressing Foxg1 expression in mouse medial pallium. J. Neurosci. 28, 10415-10421 (2008).

53. Kokubu, H. \& Lim, J. X-gal staining on adult mouse brain sections. Bio Protoc. 4, e1064 (2014).

54. Ippolito, D. M. \& Eroglu, C. Quantifying synapses: An immunocytochemistry-based assay to quantify synapse number. J. Vis. Exp. 45, 2270 (2010).

55. Fiala, J. C. Reconstruct: a free editor for serial section microscopy. J. Microsc. 218, 52-61 (2005).

56. Risher, W. C. et al. Rapid Golgi analysis method for efficient and unbiased classification of dendritic spines. PLoS ONE 9, e107591 (2014).

57. Meijering, E. et al. Design and validation of a tool for neurite tracing and analysis in fluorescence microscopy images. Cytometry A 58, 167-176 (2004).
58. Thompson, C. L. et al. A high-resolution spatiotemporal atlas of gene expression of the developing mouse brain. Neuron 83, 309-323 (2014).

59. Gong, E. et al. A gene expression atlas of the central nervous system based on bacterial artificial chromosomes. Nature 425, 917-925 (2003).

60. Elsen, G. E. et al. The protomap is propagated to cortical plate neurons through an Eomes-dependent intermediate map. Proc. Natl Acad. Sci. USA 110, 4081-4086 (2013).

61. Tournier, J.-D. et al. MRtrix: diffusion tractography in crossing fiber regions. Int. J. Imaging Syst. Technol. 22, 53-66 (2012).

62. Tournier, J. D. et al. Robust determination of the fibre orientation distribution in diffusion MRI: non-negativity constrained super-resolved spherical deconvolution. Neuroimage 35, 1459-1472 (2007).

63. Miller, J. A. et al. Transcriptional landscape of the prenatal human brain. Nature $\mathbf{5 0 8}$, 199-206 (2014).

64. Lun, M. P. et al. Spatially heterogeneous choroid plexus transcriptomes encode positional identity and contribute to regional CSF production. J. Neurosci. 35, 4903-4916 (2015).

Acknowledgements We thank S. Bai and T. Nottoli for technical help in the generation of gene-edited mouse lines; F. Hyder and J. Mihailovic for their assistance with MRI diffusion-weighted sequence design and conducting the MRIs; A. Duque for the use of equipment from MacBrainResource (MH113257); J. Rubenstein for sharing reagents; F. Li and M. Onorati for generating human iPS cells used in this study; Y. Gilad and B. Pavlovic for providing chimpanzee iPS cells; and the members of the N.S. laboratory for comments. This work was supported by the NIH (HG010898, MH106874, MH106934, MH110926 and MH116488) and the Simons Foundation Autism Research Institute (SFARI) 572080 (N.S.). The project that gave rise to these results received the support of a fellowship from the 'La Caixa' Foundation (ID 100010434). The fellowship code is LCF/BQ/PI19/11690010. Additional support was provided by the NIH T32 fellowship (MH18268) (K.P.), the National Science Foundation Graduate Research Fellowship Program (S.K.M.), the Ministerio de Ciencia e Innovación, Spain (PID2019104700GA-IOO) (G.S.), the Kavli Foundation and the James S. McDonnell Foundation (N.S.).

Author contributions M.S., K.P. and N.S. designed the research. M.S., K.P. and N.K. performed mouse experiments and analysed the data. B.L.-G. and S.K.M. analysed human and mouse transcriptomic datasets. G.S. analysed the enrichment of binding sites for RA receptors. S.-K.K. performed and analysed primate organoid and human neuronal primary culture experiments. M.S. generated the constructs for mutant mice lines. X.X. performed pronuclear injection. D.A. and K.P. analysed mouse imaging data. M.S. and A.M.M.S. performed post-mortem human and macaque tissues experiments. N.S. conceived the study. M.S., K.P. and N.S. wrote the manuscript. All authors discussed the results and implications and commented on the manuscript at all stages.

Competing interests The authors declare no competing interests.

Additional information

Supplementary information The online version contains supplementary material available at https://doi.org/10.1038/s41586-021-03953-x.

Correspondence and requests for materials should be addressed to Kartik Pattabiraman or Nenad Sestan.

Peer review information Nature thanks Alex Pollen and the other, anonymous, reviewer(s) for their contribution to the peer review of this work.

Reprints and permissions information is available at http://www.nature.com/reprints. 

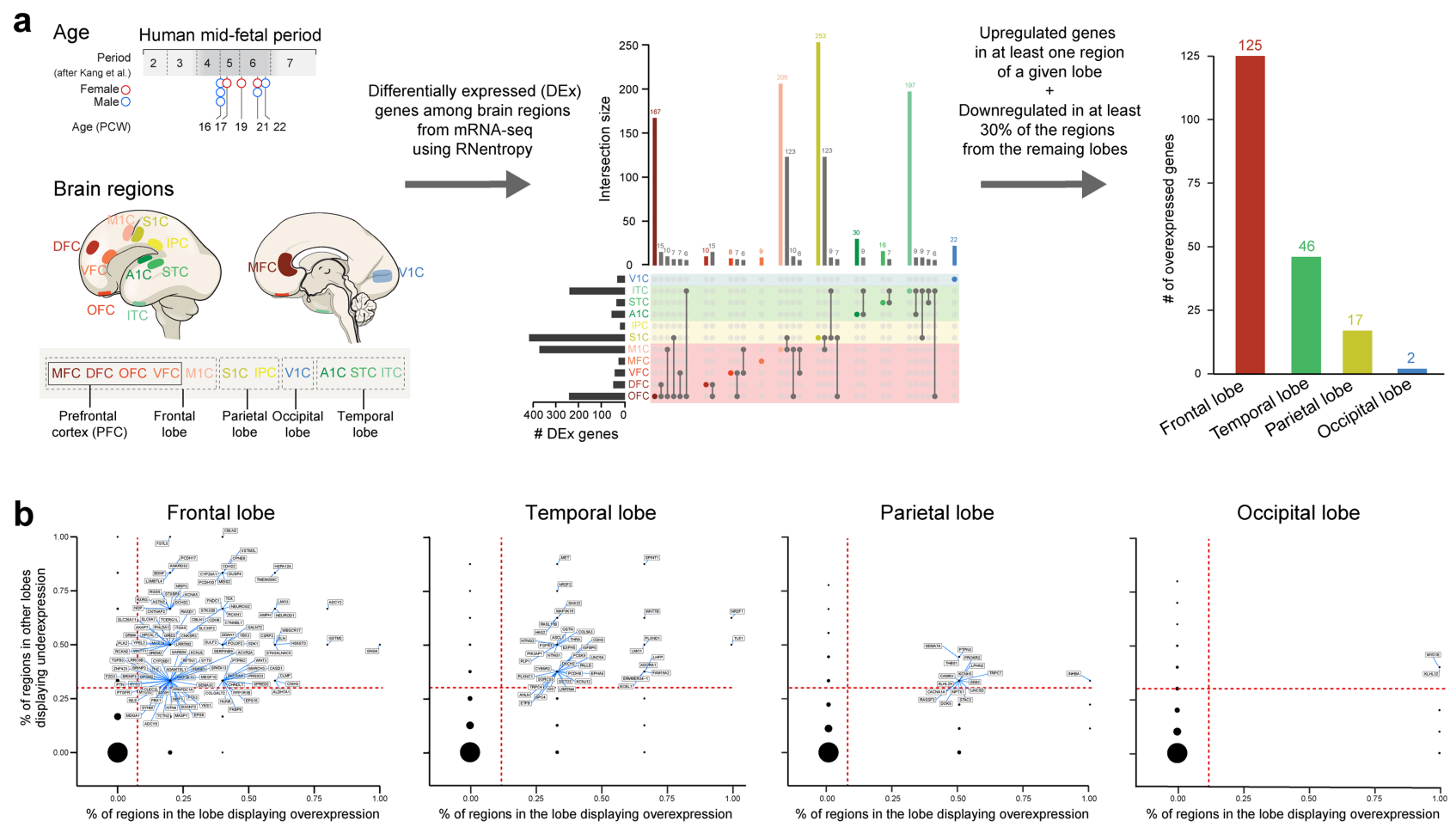

C

Human midfetal period

Gene loadings for PC1

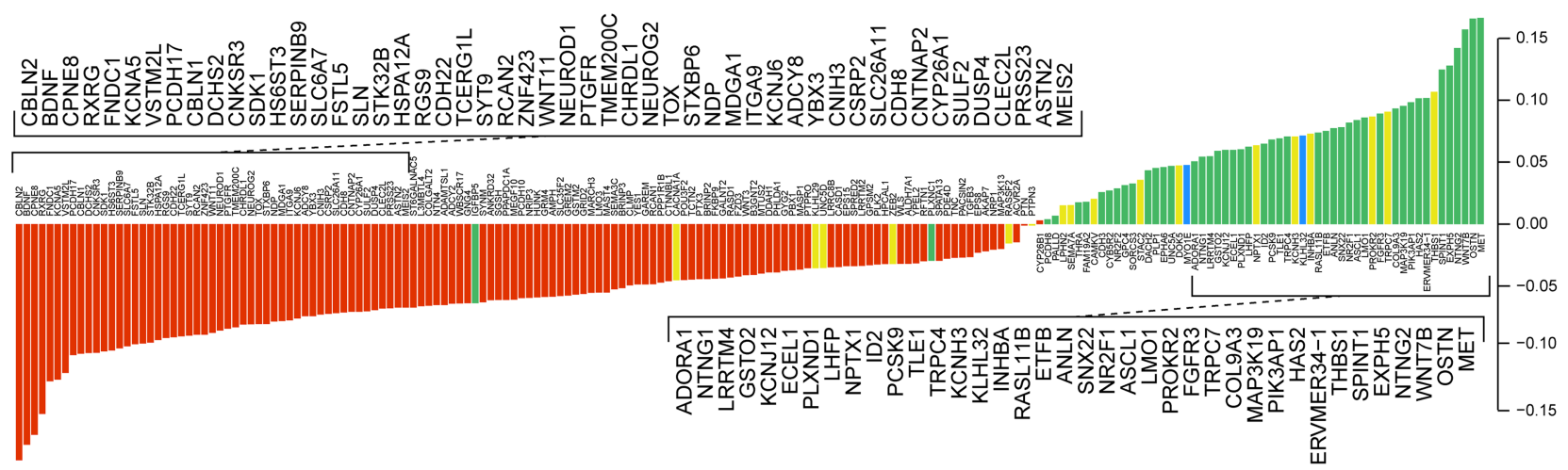

d Macaque midfetal period

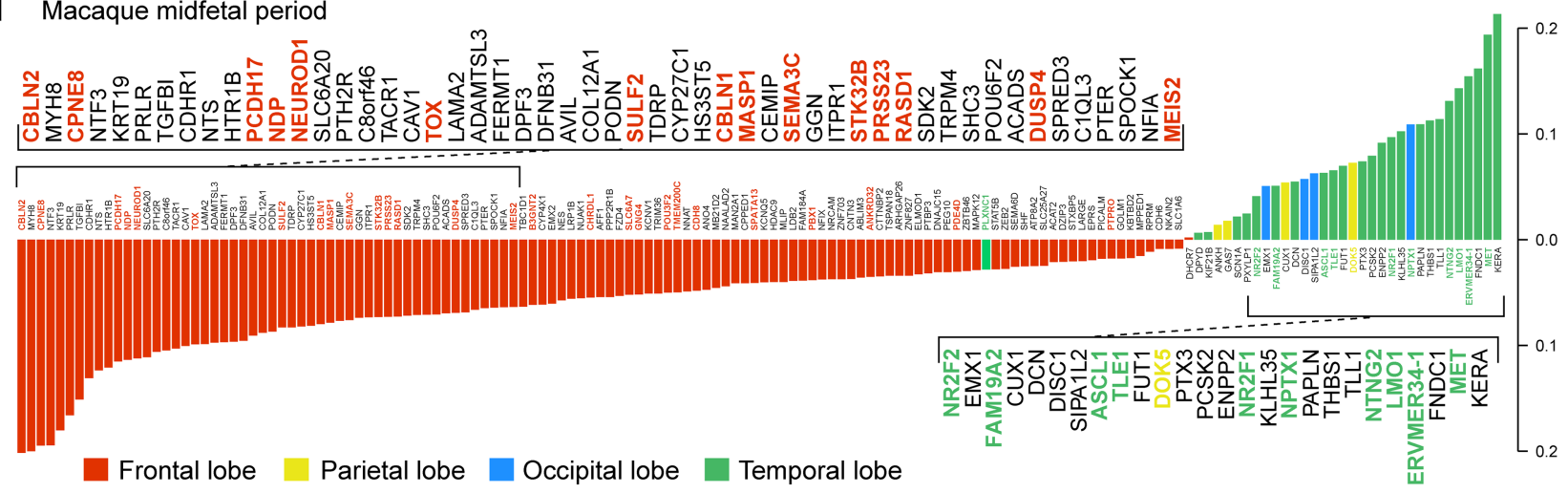

Extended Data Fig. 1 |See next page for caption. 


\section{Article}

Extended Data Fig. 1 | Workflow for analysis of mid-fetal RNA-seq data and genes upregulated in individual lobes. a, Human developmental neocortical RNA-seq dataset and workflow for analysis to identify genes upregulated in each cortical lobe.b, Genes upregulated in the frontal, temporal, parietal and occipital lobes in comparison to the other lobes. We identified 190 proteincoding genes that are specifically upregulated, using stringent criteria, in at least one area within a lobe in comparison with areas from other lobes: 125 in the frontal lobe, 46 in the temporal lobe, 17 in the parietal lobe, and 2 in the occipital lobe. The X-axis represents proportion of putative areas in the frontal lobe in which the gene is significantly upregulated according to RNentropy. The Y-axis represents proportion of areas in the other lobes in which the gene is significantly downregulated according to RNentropy. Upregulated genes, the ones delimited by dashed red lines, are labelled. c, d, Gene loadings of PC1 from PCA of protein-coding genes that are specifically enriched in one of the four lobes of the mid-fetal human (c) and macaque (d) cortex. Colours represent the cortical lobe where the gene was found to be specifically upregulated. For reproducibility information, see Methods. 

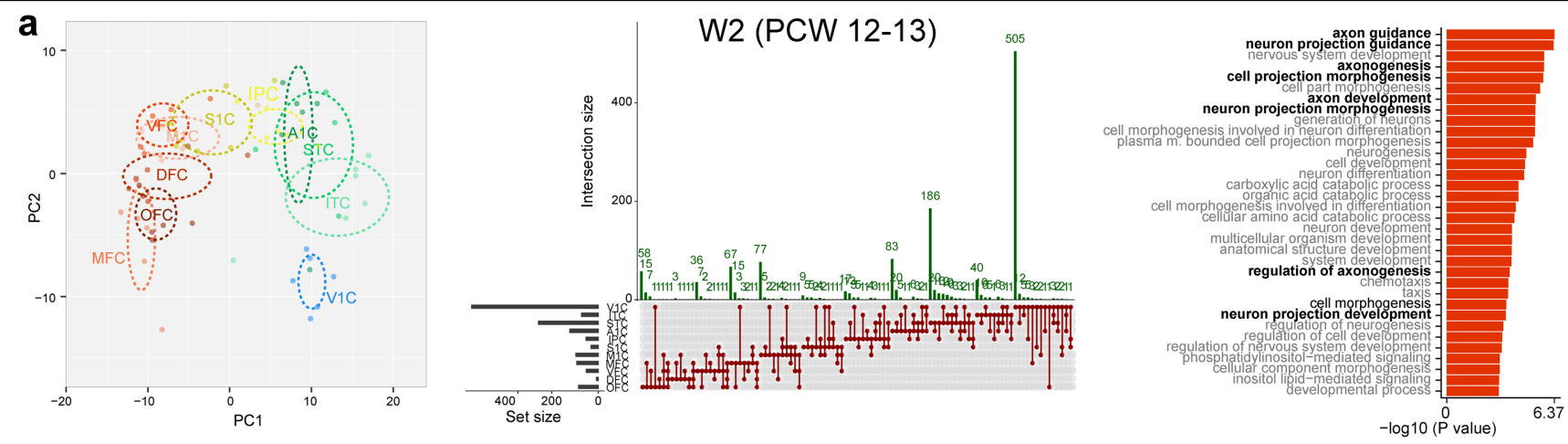

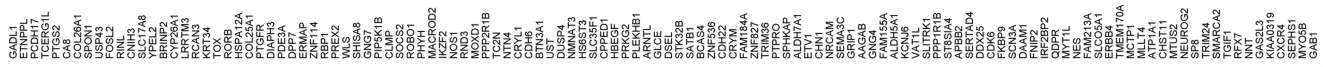
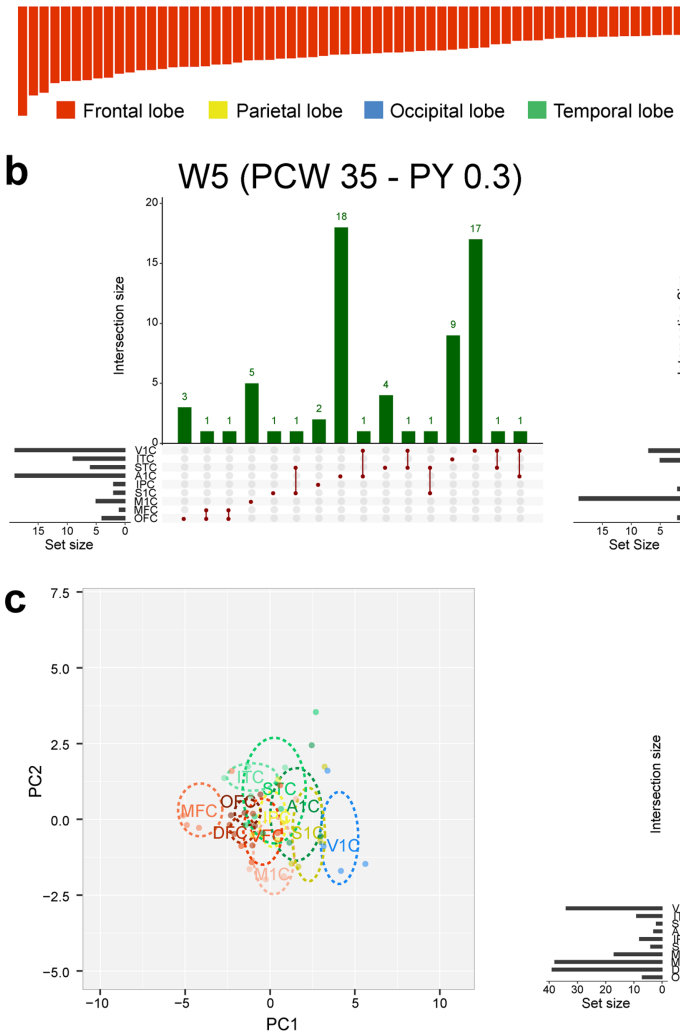

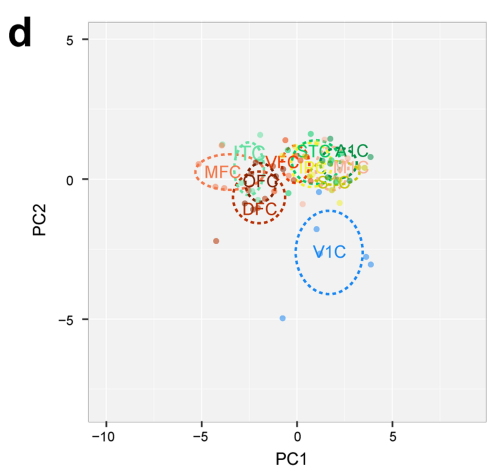

Extended Data Fig. 2 | Analysis of genes upregulated in individual cortical lobes during other developmental periods. a, Analysis of upregulated genes during window 2 (early fetal period, PCW 12-13 specimens in the BrainSpan datase ${ }^{17}{ }^{17}$. See Fig. 1a for further explanation. $\mathbf{b}$, Number of upregulated genes
Gene loadings for PC1
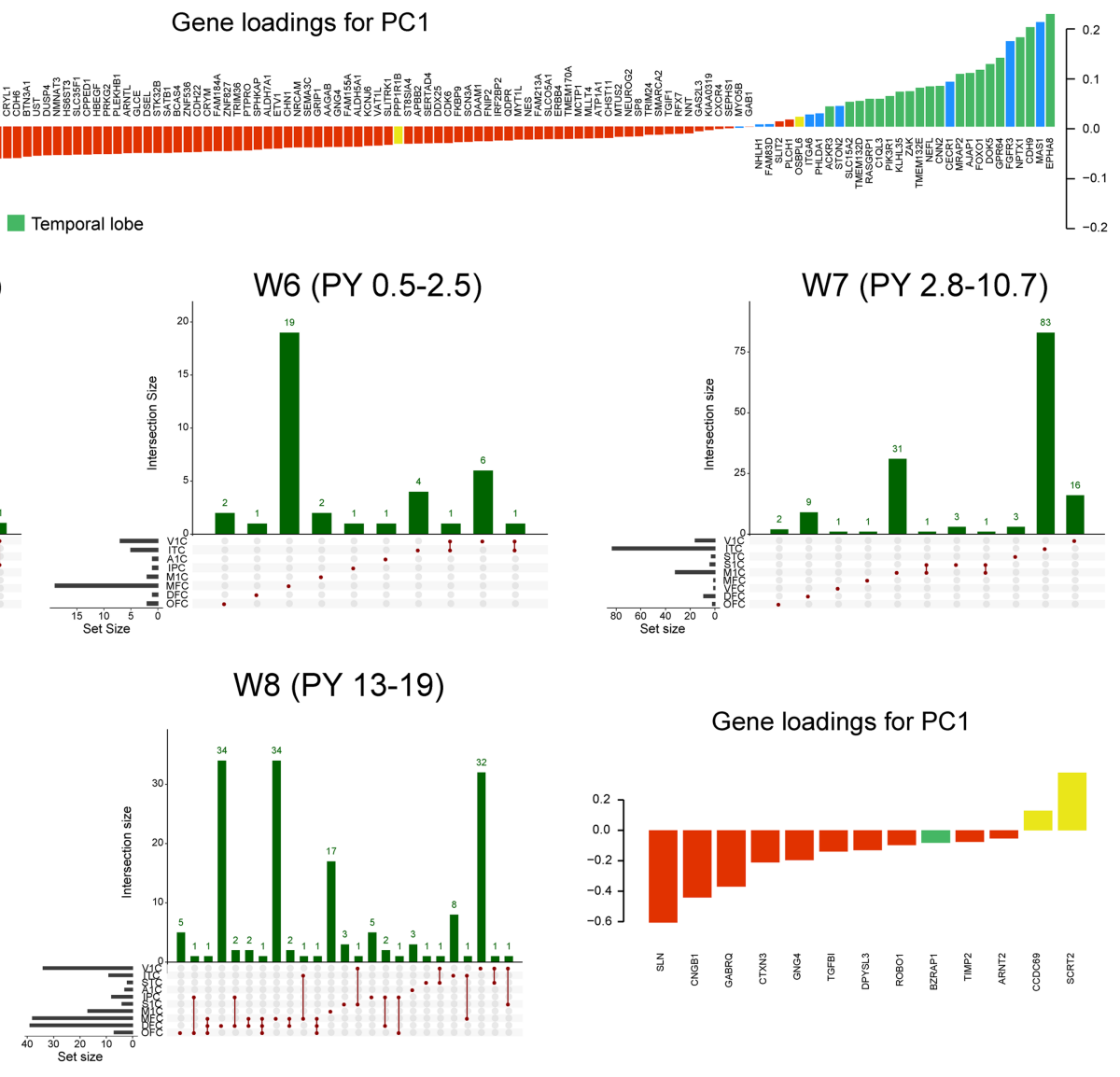

W7 (PY 2.8-10.7)

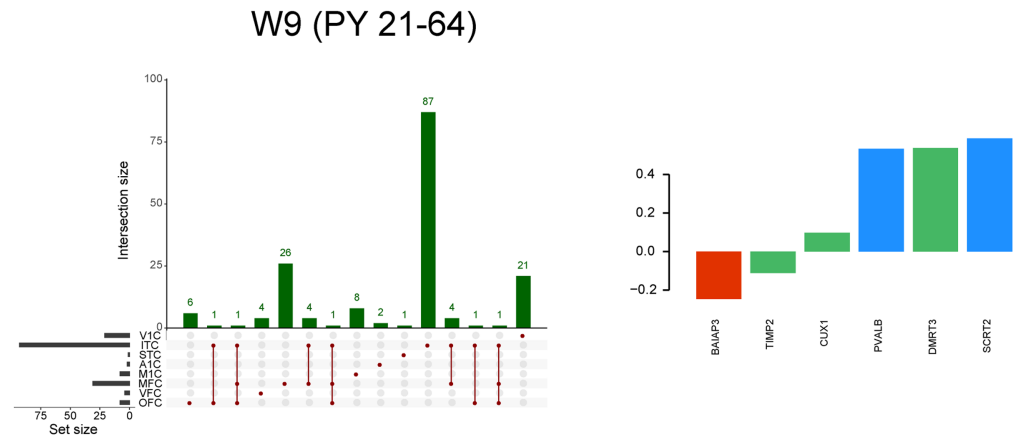

during late prenatal, and early postnatal stages in individual lobes are significantly reduced compared to earlier stages. c, d, Analysis of upregulated genes during window 8 (postnatal year, PY 13-19) and W9 (PY 21-64). See Fig. 1a for further explanation. For reproducibility information, see Methods. 
a

Response to retinoic acid

Cellular response to retinoic acid

Retinoic acid receptor signaling pathway

Retinoic acid biosynthetic process

Retinoic acid metabolic process

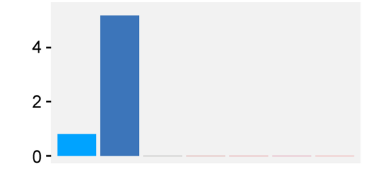

Regulation of retinoic acid receptor signaling pathway
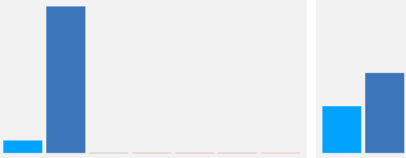

Negative regulation of retinoic acid receptor signaling pathway

Positive regulation of retinoic

Retinoic acid catabolic process

9-cis-retinoic acid biosynthetic process

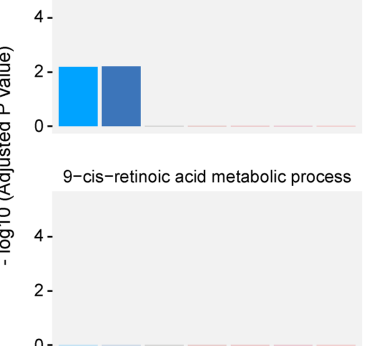

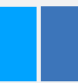

Regulation of retinoic acid biosynthetic process
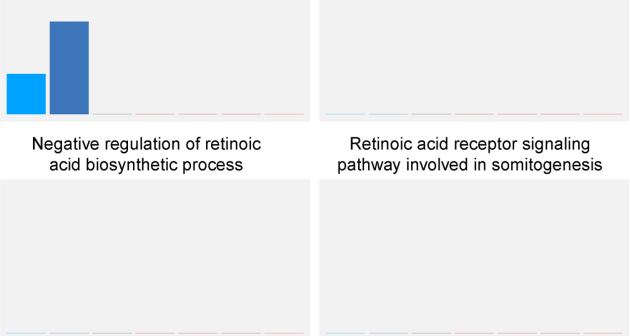

Retinoic acid receptor signaling pathway involved in somitogenesis

Positive regulation of retinoic acid biosynthetic process

Retinoic acid receptor binding

Retinoic acid binding

Retinoic acid 4-hydroxylase activity

Retinoic acid-responsive element binding

All-trans retinoic acid 3,4-desaturase activity

4 -

2 -

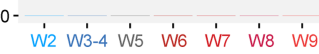
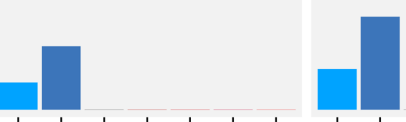

W2: PCW 12-13 W3-4: PCW 16-22 W5: PCW 35 - PY 0.3

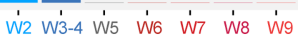
(Early fetal dev.) (Mid-fetal dev.)

W6: PY $0.5-2.5$

(Late infancy and early childhood)

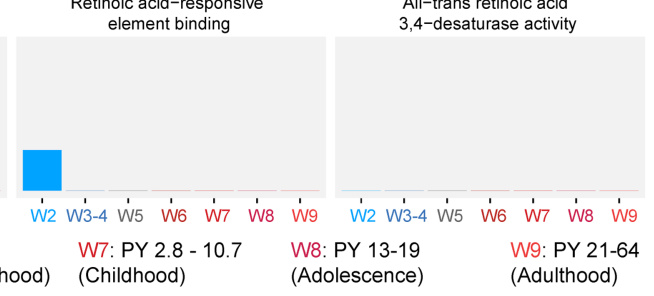

b
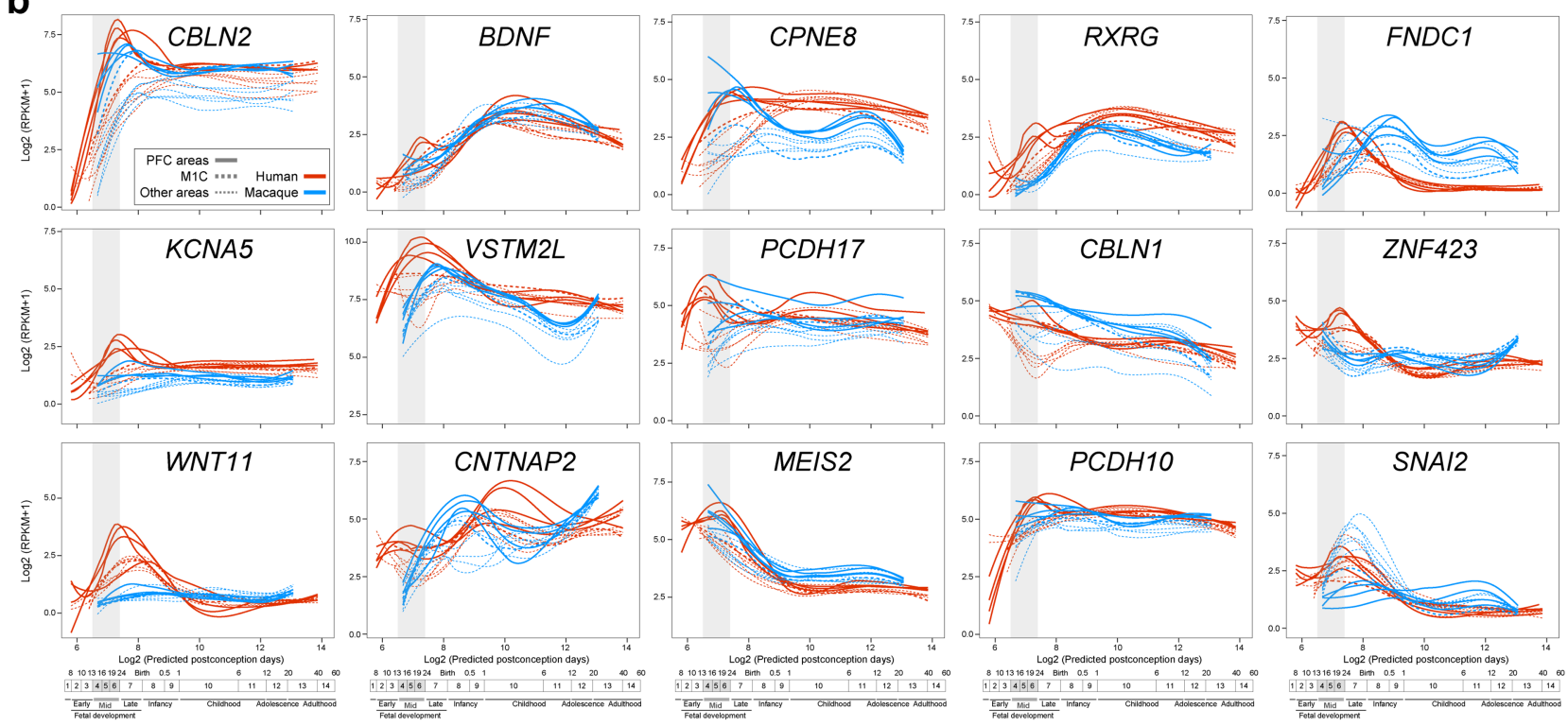

\section{Extended Data Fig. 3 | Extended RA-specific GO analysis and} spatiotemporal expression of select genes upregulated in the mid-fetal frontal lobe. a, Analysis for statistically significant enrichment of upregulated genes during developmental and adult stages for GO terms associated with RA. $\mathrm{X}$-axis represents windows analysed, which are defined at the bottom of the figure. b, Spatiotemporal expression of select genes upregulated in the mid-fetal frontal lobe from Fig. 1 in sixteen neocortical areas across human (red) and macaque (blue) development using BrainSpan (brainspan.org) and PsychENCODE (evolution.psychencode.org) RNA-seq data ${ }^{17,19}$. Thick full lines represent four PFC areas, thick dotted line represents the primary motor cortex (M1C) and thin dotted lines represent the other non-frontal neocortical areas. Vertical grey box demarcates mid-fetal periods analysed in Fig. 1. Timeline of human and macaque development and the associated periods designed by Kang et $\mathrm{al}^{39}$. shown below. Predicted ages were calculated using the TranscriptomeAge algorithm ${ }^{19}$, which aligns our earliest macaque samples (PCD 60) with human early mid-fetal samples. Distinct global patterns of spatiotemporal expression were observed. For example, precocious expression in the frontal lobe/PFC followed by broad expression in all eleven neocortical areas (e.g. $C B L N 2, B D N F$ ), transient enrichment in the frontal lobe/ PFC (e.g. WNT11, PCDH17) and downregulation in non-PFC areas during mid-fetal development (e.g. MEIS2). 
a

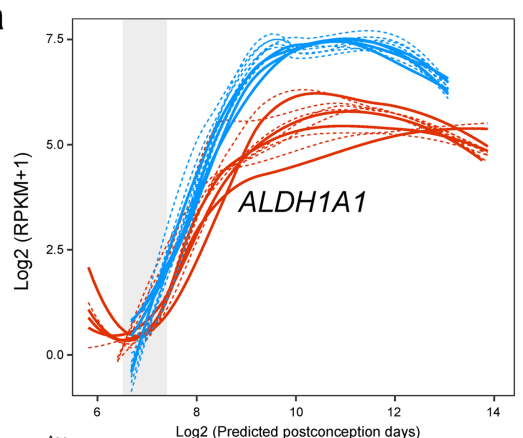

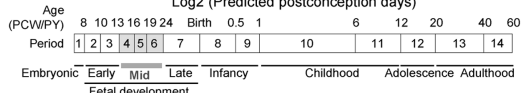

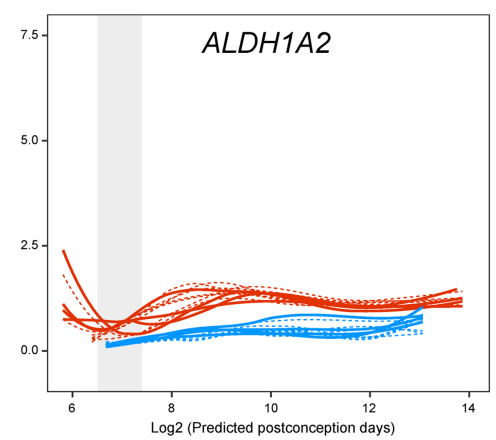

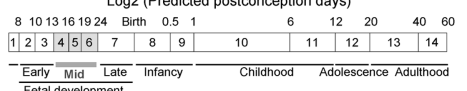

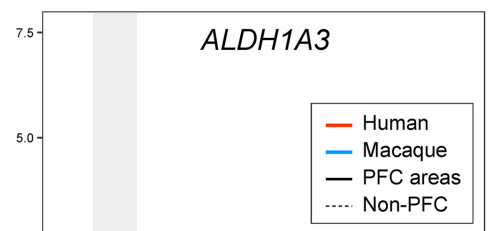

b

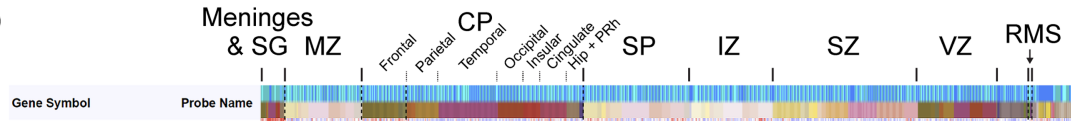

Meninges

ame

ALDHAA

A_23_P P83098

ALDHAA

ALDH1A2
ALDH1A2

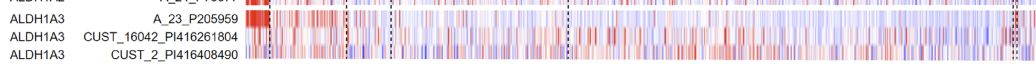

ALDHA3
ALDHA3
ALDH1A3

C

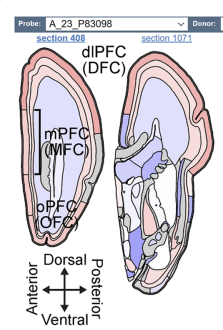

ALDH1A1

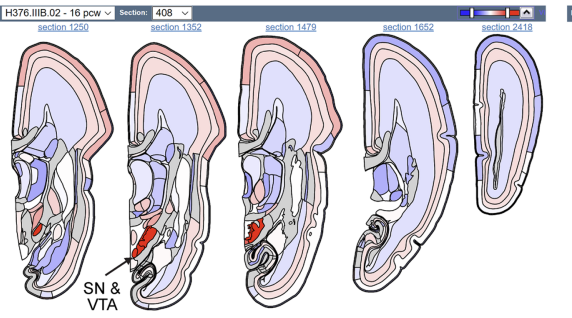

d

Human
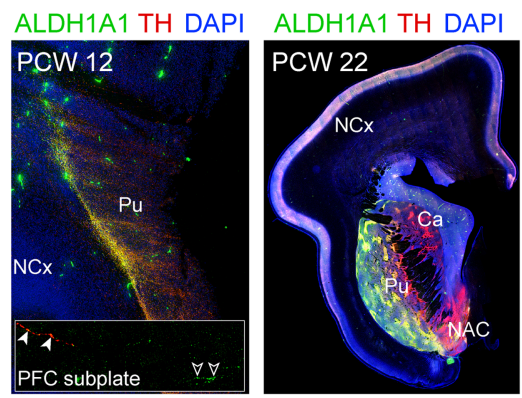

f
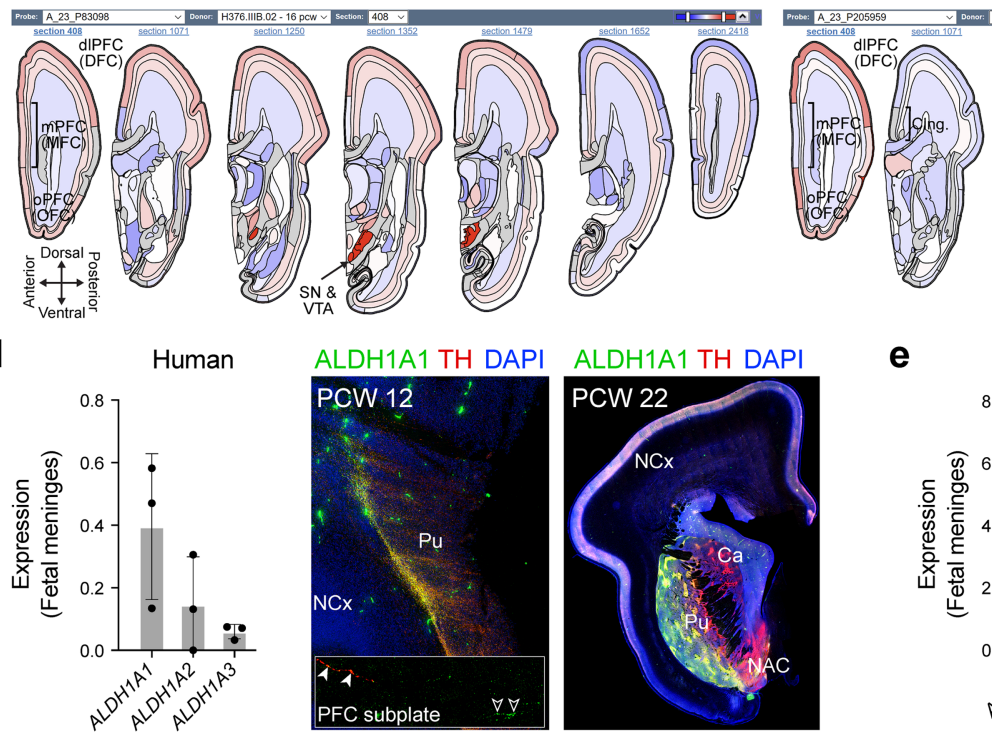

$A L D H 1 A 3$

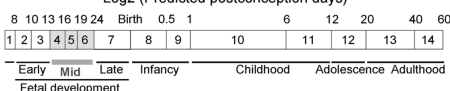

SN, VTA ChP RF ${ }_{\downarrow} \mathrm{CBC}$ I

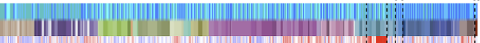
НИН U.

e

\section{ALDH1A3 ISH}

Human (PCW 21)
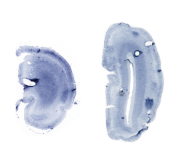

\begin{tabular}{ll|l} 
& mPFC & Occ \\
Macaque (PCD 110) & $\mathrm{cP}$ & $\mathrm{CP}$ \\
& $\overline{\mathrm{sz}}$ & $\overline{\mathrm{sz}}$
\end{tabular}

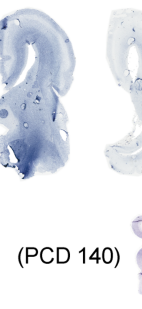

Mouse (PD 0)

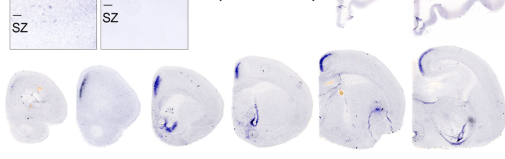

Anterior

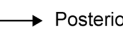

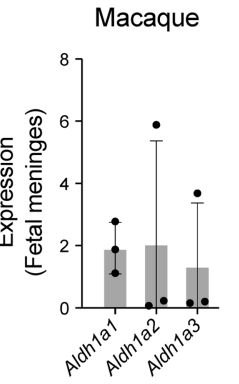
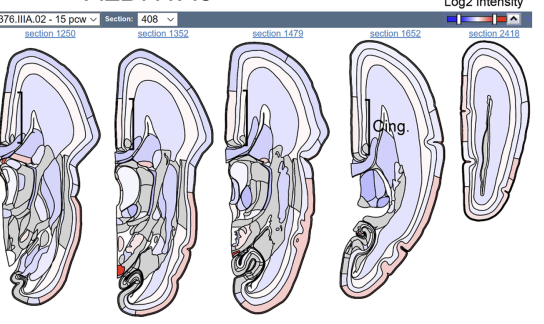

ALDH1A1 TH DAPI

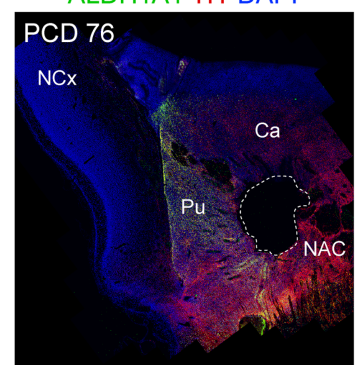

g

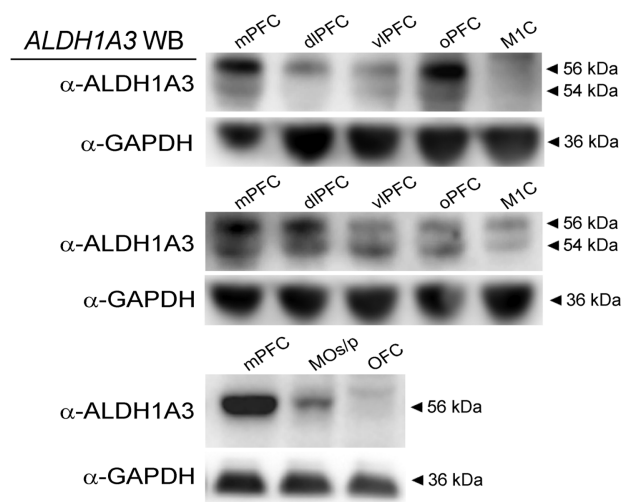

Extended Data Fig. 4 | See next page for caption. 


\section{Article}

Extended Data Fig. 4 | Expression of RA-synthesizing enzymes in the developing human and macaque cortex. a, Spatiotemporal expression of genes encoding RA synthesizing enzymes, $A L D H 1 A 1,2$ and 3 , in eleven neocortical areas of human and macaque during prenatal and postnatal development using BrainSpan (brainspan.org) and PsychENCODE (evolution. psychencode.org) RNA-seq data ${ }^{17,19}$. Red and blue lines indicate human and macaque, respectively, and dotted lines represent the non-PFC expression in the PFC plot. Vertical grey box demarcates mid-fetal developmental periods. Predicted ages, timeline of human and macaque development, and the associated periods are shown below ${ }^{19,63} \cdot \mathbf{b}$, Heat map of normalized (z-score) microarray signals computed for genes encoding RA synthesizing enzymes from the BrainSpan human prenatal laser microdissection microarray data ${ }^{63}$ (brainspan.org). Left columns represent gene name and specific probe. Each column represents regions of the brain labelled above the heat maps. Darker reds represent high expression levels. c, Anteroposterior visual representation of human ALDH1A1 and ALDH1A3 expression at PCW 16 and 15 respectively from BrainSpan atlas ${ }^{58}$. $A L D H 1 A 1,2$ and 3 expression in mid-fetal human (PCW 19, 19, 20) (d, left) and macaque (PCD 80, 80, 110) (e, left) meninges. Immunostaining for ALDH1A1 and TH in human PCW 12 and 22 brains (d, right), and in macaque PCD 76 brain (e, right). White arrowheads, and open arrowheads indicate ALDH1A1+;TH+ and ALDH1A1+;TH-axons in the subplate, respectively. NCX, neocortex; PU, putamen; NAC, nucleus accumbens; CA, CA subfields of hippocampus. Errors bars: S.D. $\mathrm{N}=3 . \mathbf{f}$, Anterior to posterior expression of ALDH1A3 mRNA in human (PCW 21), macaque (PCD 140) and mouse (PD 0) brain. $\mathrm{N}=2$ for human and macaque, $\mathrm{N}=3$ for mouse. $\mathrm{g}$, Western blot using ALDH1A3 antibody in human (PCW 20), macaque (PCD 114) and mouse (PD 0) frontal cortex areas. In macaque and human, there are two bands that are likely to represent ALDH1A3 (56 kDa) and ALDH1A1 ( $54 \mathrm{kDa})$. Experiments were repeated at least two times for each animal species. See Extended Data Fig. 6a for schemas of frontal areas. 


\section{a}
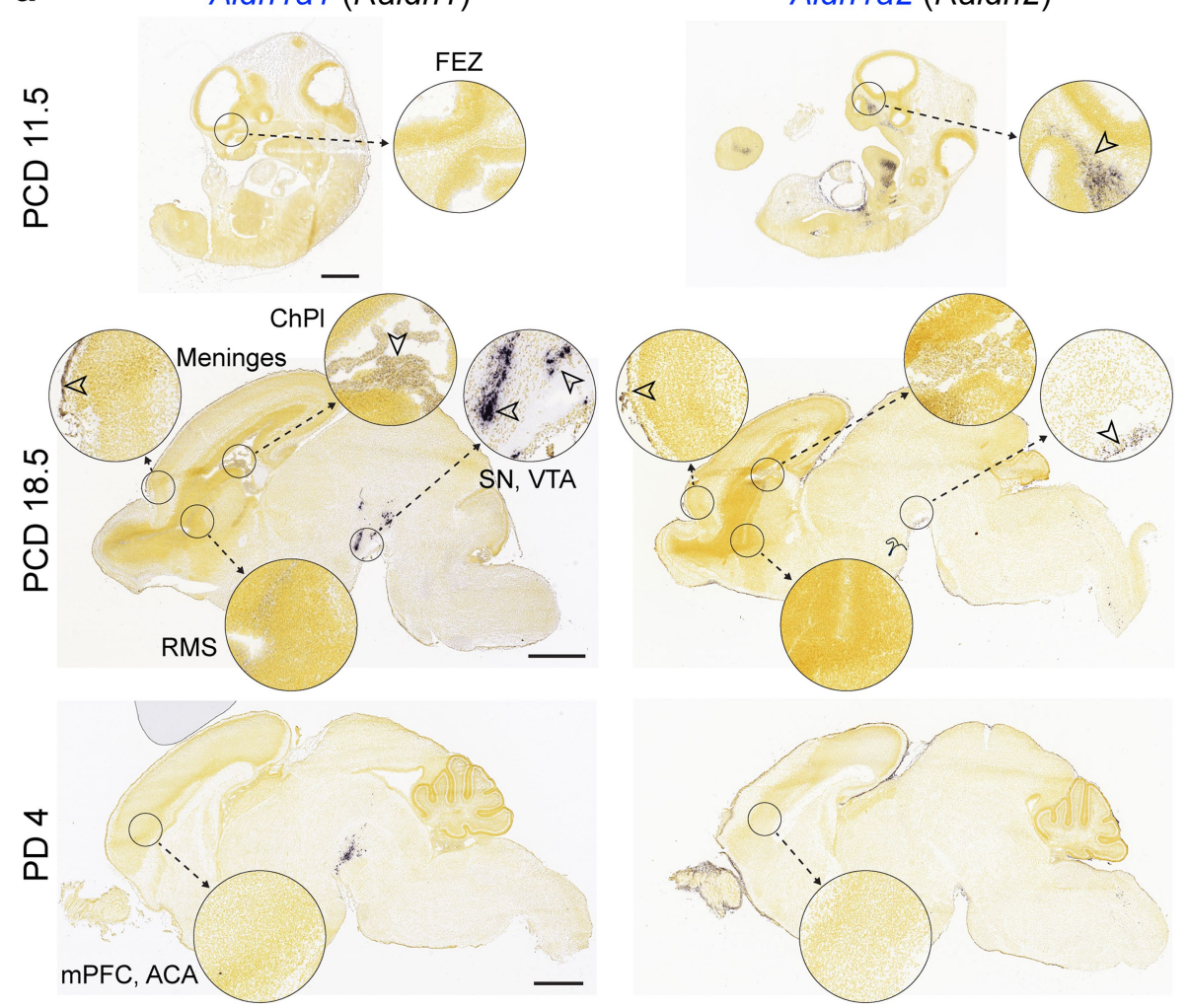

b Rara (PCD 15.5)
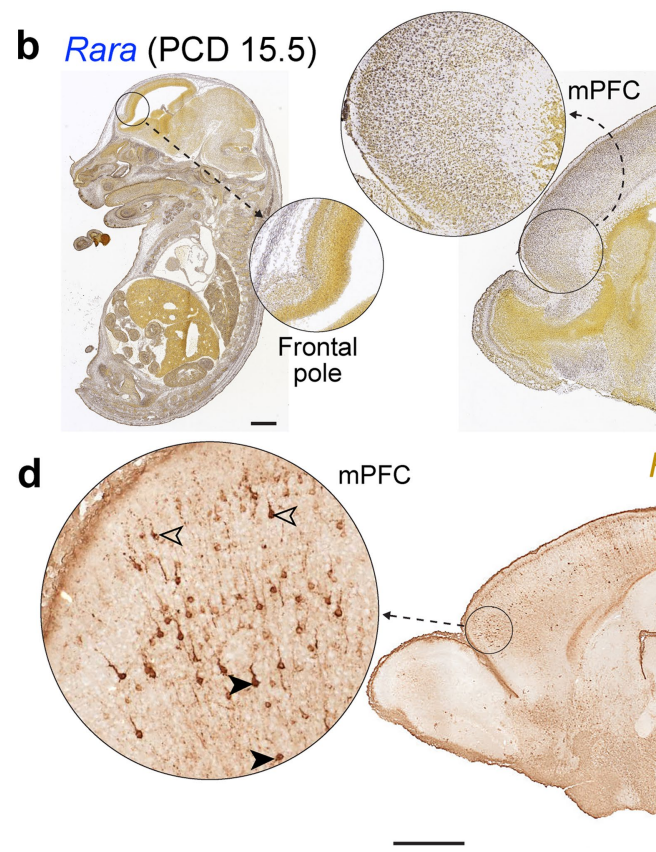

Extended Data Fig. 5 | Expression of genes encoding RA-synthesizing enzymes in the developing mouse cortex. Expression of Aldh1a1, Aldh1a2 and Aldh1a3 at PCD 11.5, 18.5 and PD 4 (a) and Rara at PCD 15.5 and PD 4 (b) from the Allen Developing Mouse Brain Atlas ${ }^{58}$ (developingmouse.brain-map.org). c, Expression of RA related genes in the PCD 18.5 choroid plexus (CP). Blue bars represent lateral ventricle $C P$ and orange bars represent fourth ventricle $C P$.
Aldh1a3 (Raldh3)
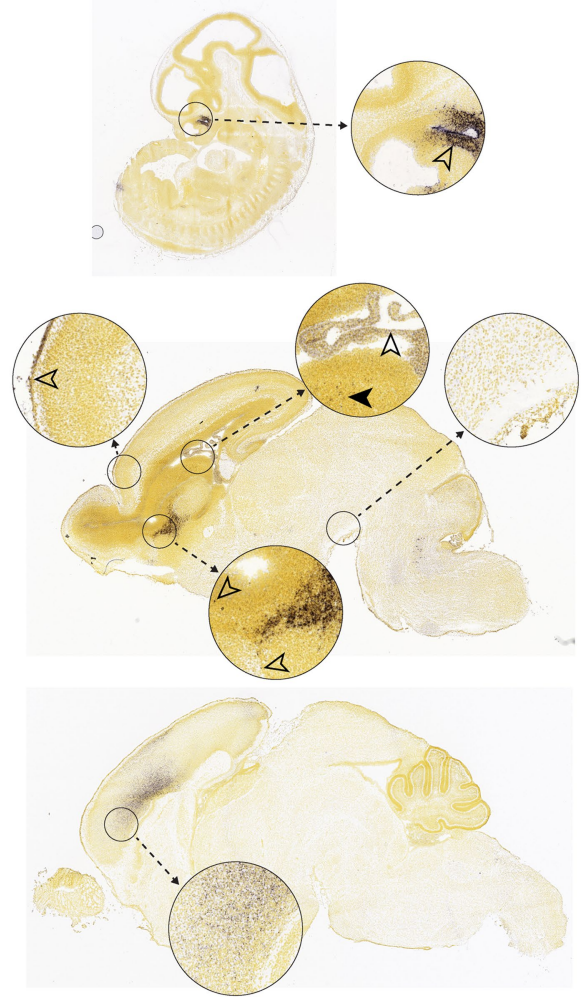

C

Lateral ventricle (telencephalic) choroid plexus Fourth ventricle (hindbrain) choroid plexus

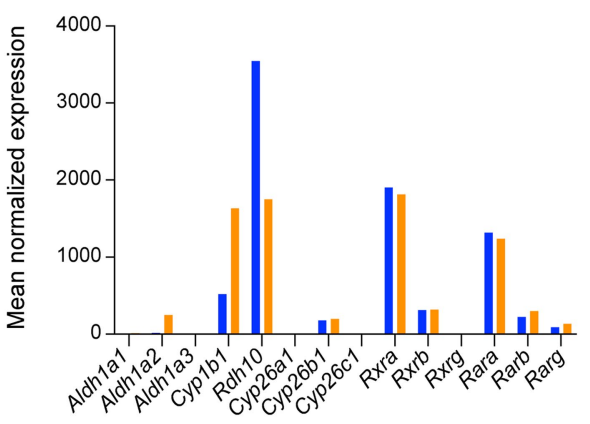




\section{Article}

a Human frontal cortex (PCW 21)

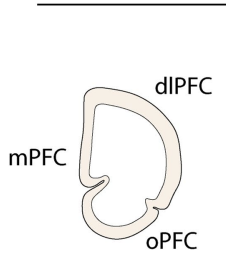

ALDH1A

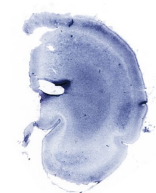

mPFC

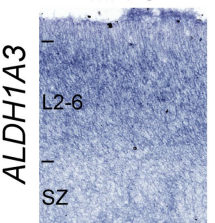

dIPFC
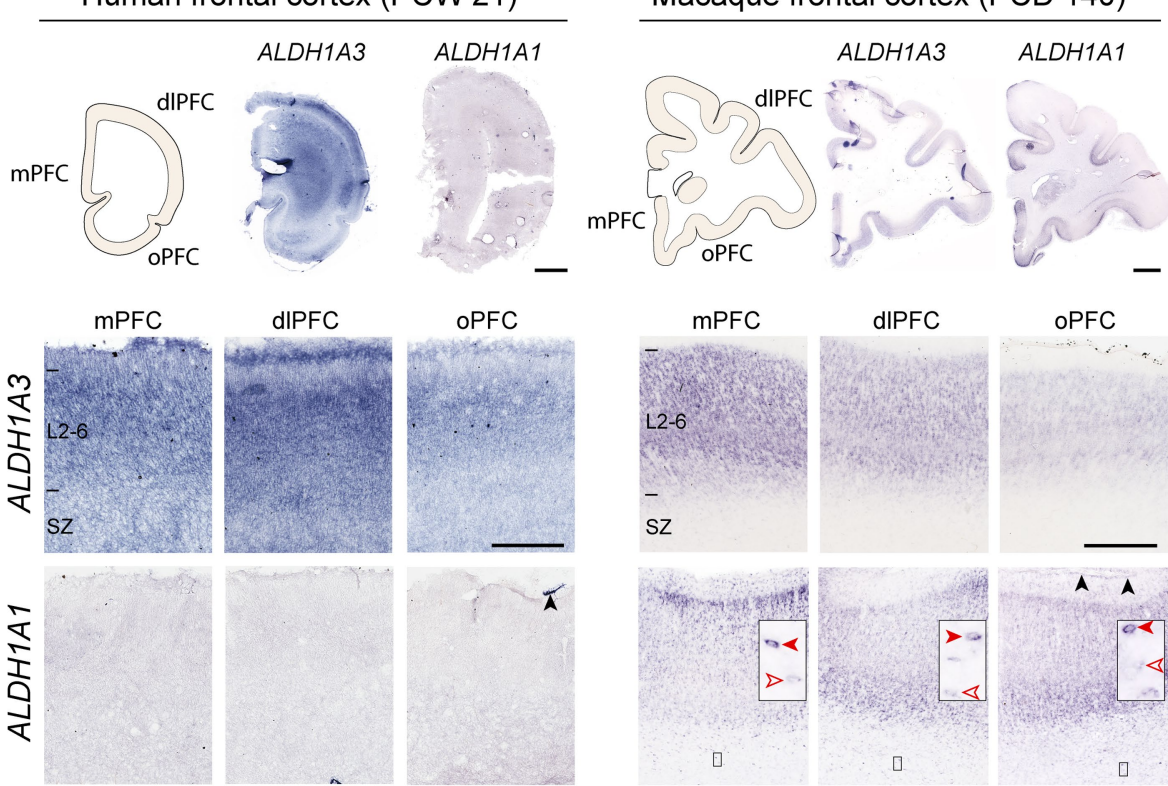

oPFC
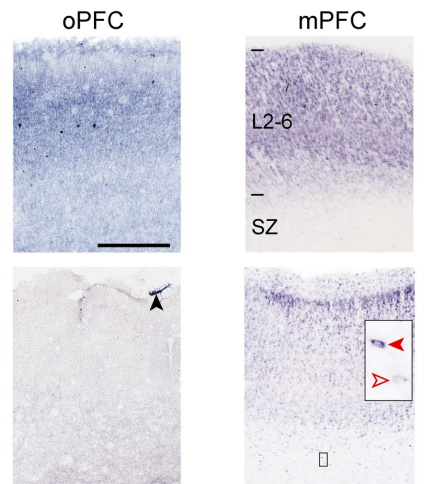

dIPFC

oPFC
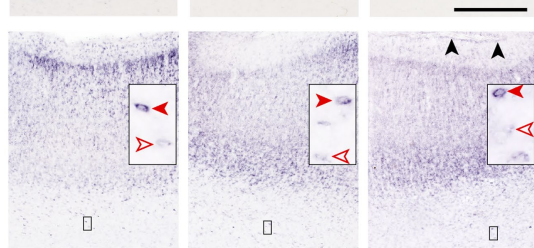
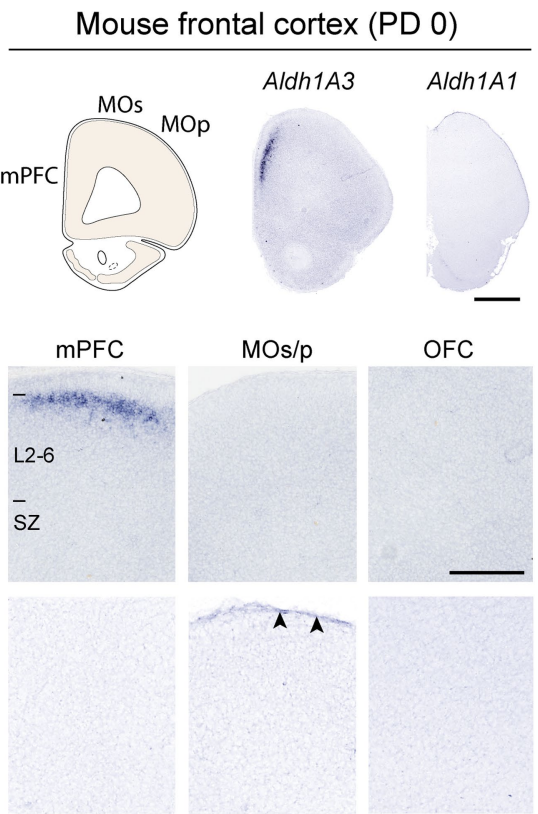

Mouse frontal cortex

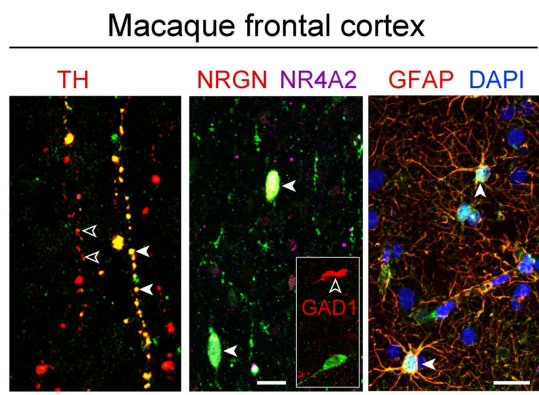

mPFC (PCD 76)

dIPFC (Adult)

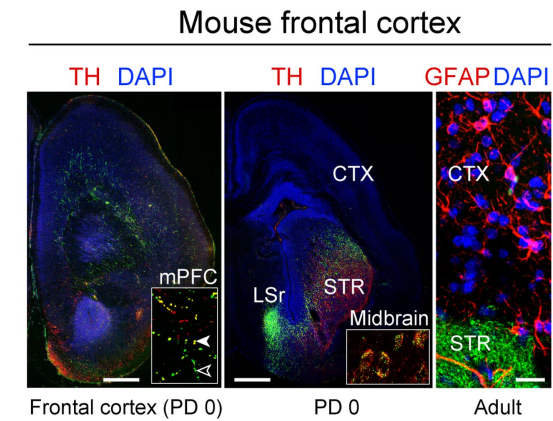

C white matter

indicate GFAP+;ALDH1A1+ astrocytes. Macaque: left, solid and open arrowheads indicate TH+;ALDH1A1+ and TH+;ALDH1A1- axons, respectively; middle, solid and open arrowheads indicate putative excitatory

NRGN+;NR4A2+;ALDH1A1+ and inhibitory GAD1+;ALDH1A1-subplate neurons, respectively; right, arrowheads indicate GFAP+;ALDH1A1+ astrocytes. Mouse: left, solid and open arrowheads indicate TH+;ALDH1A1+ and TH+;ALDH1A1mPFC axons (inset), respectively; middle, $\mathrm{TH}+$ (red) and ALDH1A1+ (green) midbrain neurons and axons in striatum (STR), lateral septal nucleus (LSR) and cortex (CTX); right, GFAP+;ALDH1A1- astrocytes. $\mathrm{N}=3$. Scale bars: $20 \mu \mathrm{m}$ (human, macaque, and mouse left and middle panels); $1 \mathrm{~mm}$ (mouse right panel). For reproducibility information, see Methods. 

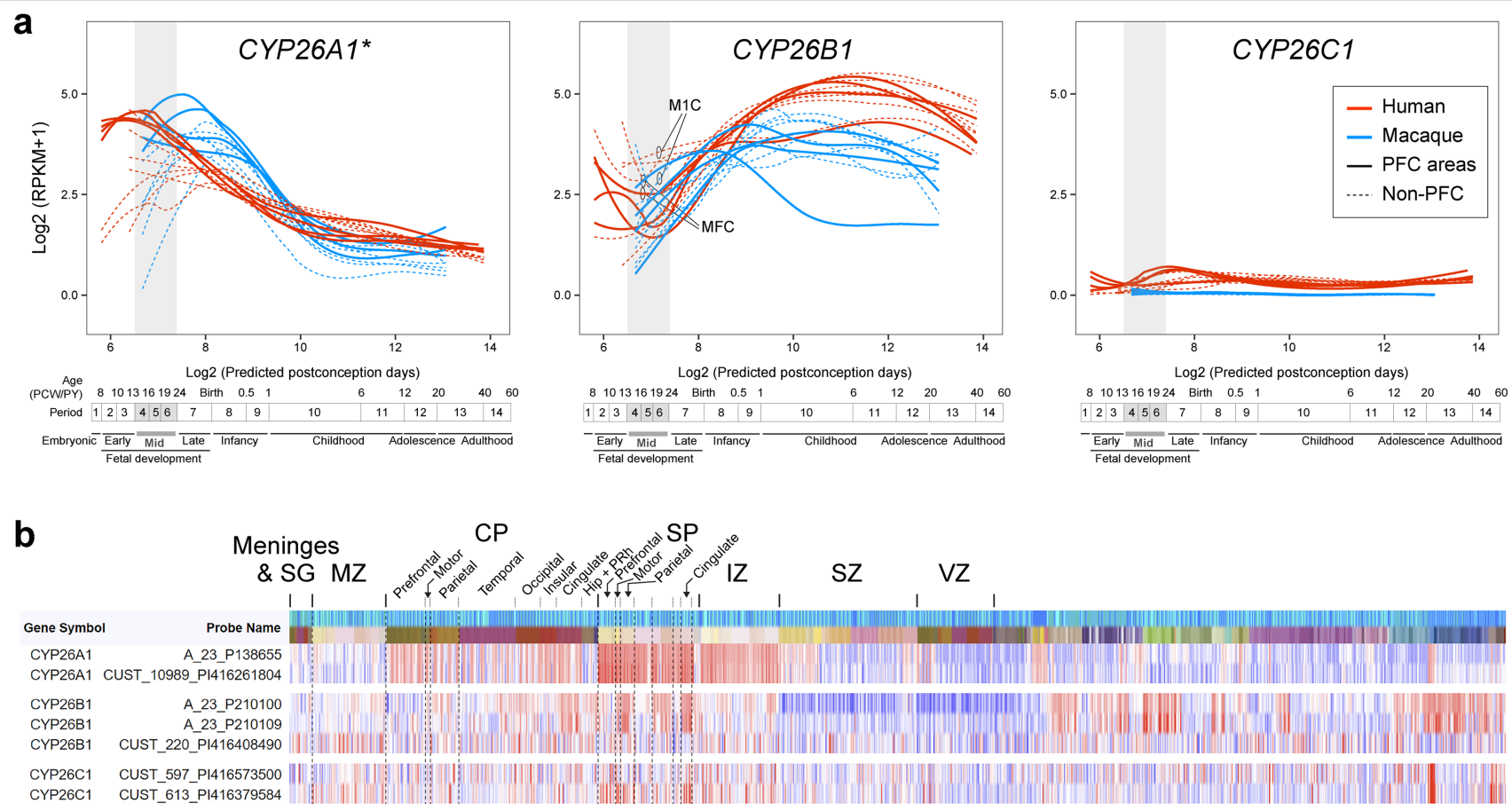

C

CYP26A1

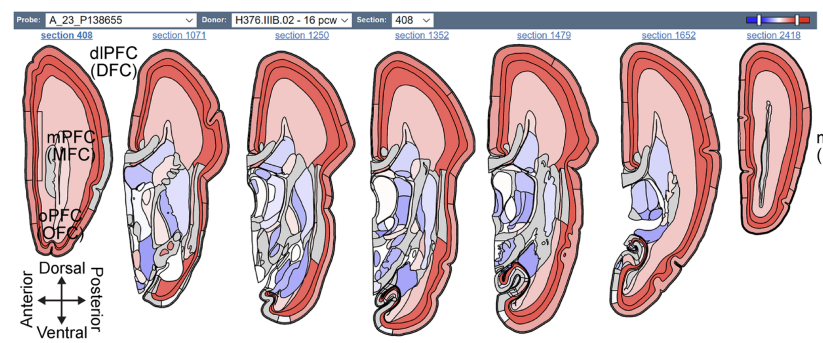

Extended Data Fig. 7 | Expression of genes encoding RA-degrading enzymes in the developing human and macaque cortex. a, Expression of RA degrading enzymes in individual regions of the cerebral cortex of human and macaque during development. Red and blue lines indicate human and macaque, respectively, and dotted lines represent the non-PFC expression in the PFC plot and vice versa. Vertical grey box demarcates mid-fetal developmental periods. Predicted ages, timeline of human and macaque development, and the associated periods are shown below ${ }^{19,39} .{ }^{*}$ Data for CYP26A1 was not present in Zhu et $\mathrm{a}^{19}$. and the data analysed individually. The
CYP26B1

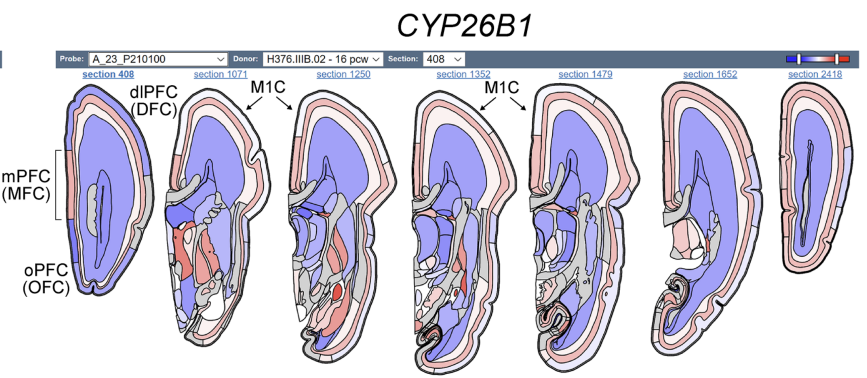

expression level for human and macaque were not normalized and can't be directly compared. b, Heat map of normalized (z-score) microarray signals computed for genes encoding RA degrading enzymes from the BrainSpan human prenatal laser microdissection microarray data ${ }^{63}$ (brainspan.org). Left column represents gene and specific probe. Rows represent regions of the brain. Darker reds represent high expression levels.c, Anteroposterior visual representation of human CYP26A1 and CYP26B1 expression at PCW 15 respectively from the BrainSpan atlas. 


\section{Article}

a

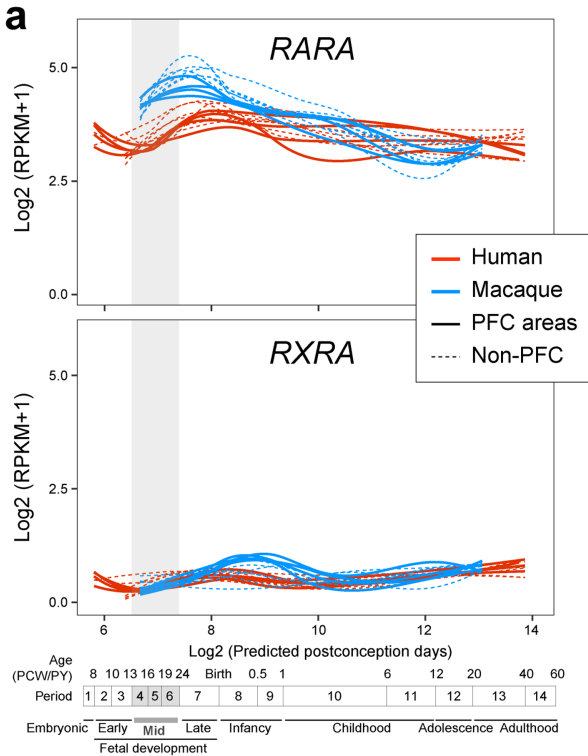

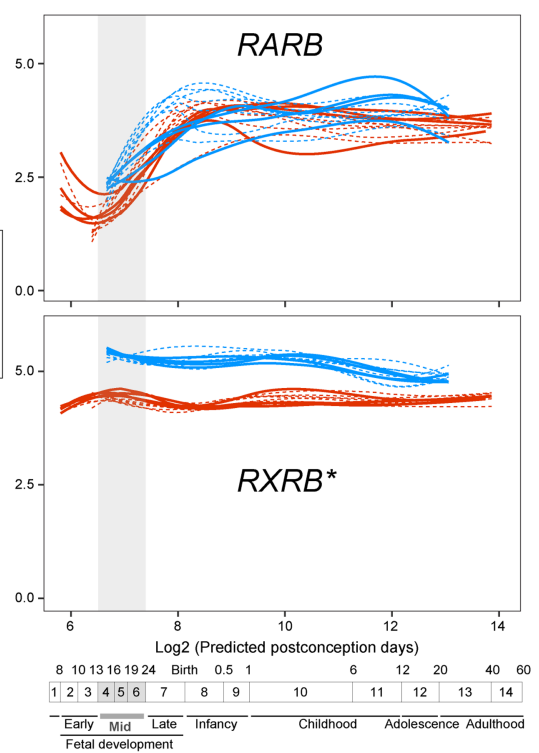

b

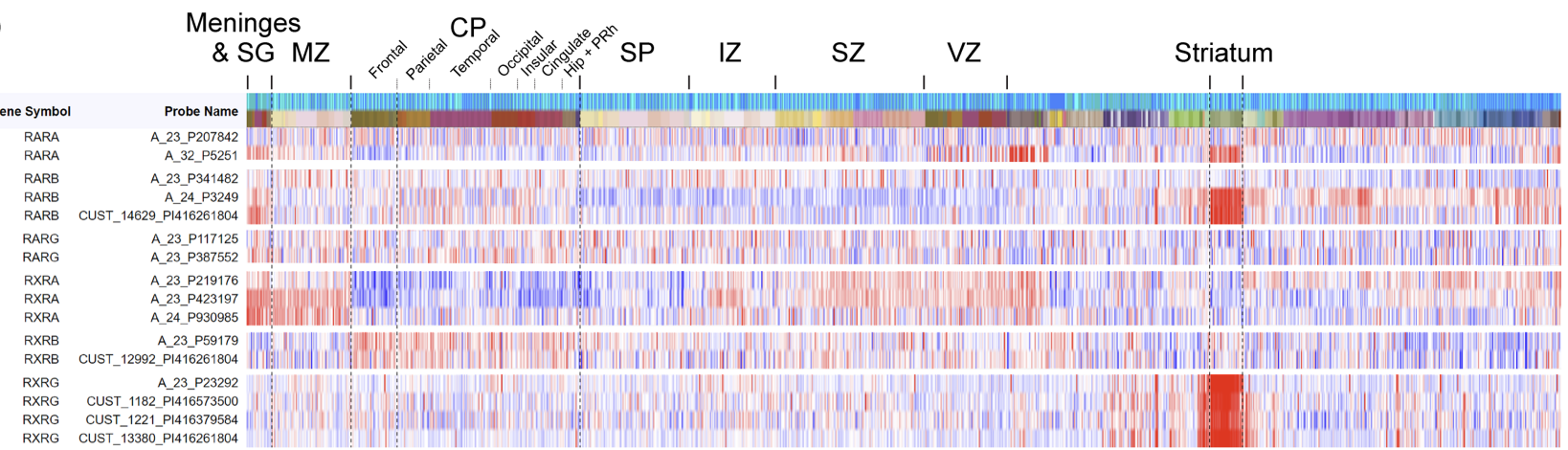

Extended Data Fig. 8 | Expression of genes encoding RA receptors in the developing human and macaque cortex. a, Expression of genes encoding RA receptors in individual regions of the cerebral cortex of human and macaque during prenatal and postnatal development. Red and blue lines indicate human and macaque, respectively, and dotted lines represent the non-PFC expression in the PFC plot and vice versa. Vertical grey box demarcates mid-fetal developmental periods. Predicted ages, timeline of human and macaque development, and the associated periods are shown below ${ }^{19,39} \cdot{ }^{*}$ Data for $R X R B$ was not present in Zhu et al ${ }^{19}$ and the data were analysed individually. The expression level for human and macaque were not normalized and cannot be compared. b, Heat map of normalized (z-score) microarray signals computed for genes encoding $\mathrm{RA}$ receptors from the BrainSpan human prenatal laser microdissection microarray data ${ }^{63}$ (brainspan.org). Left column represents gene and specific probe. Rows represent regions of the brain. Darker reds represent high expression levels. 
a

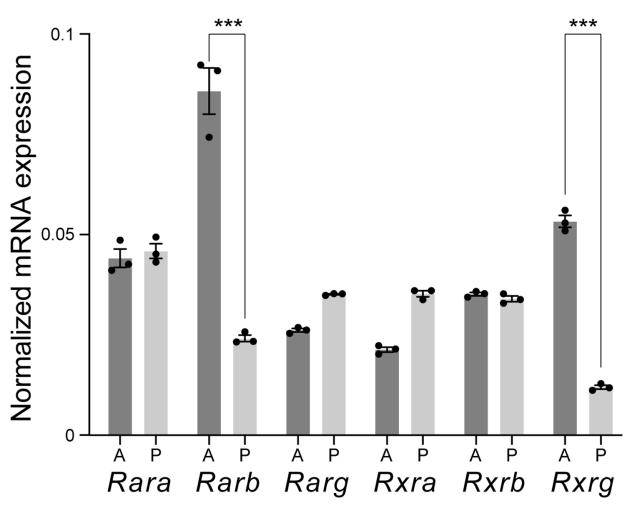

C
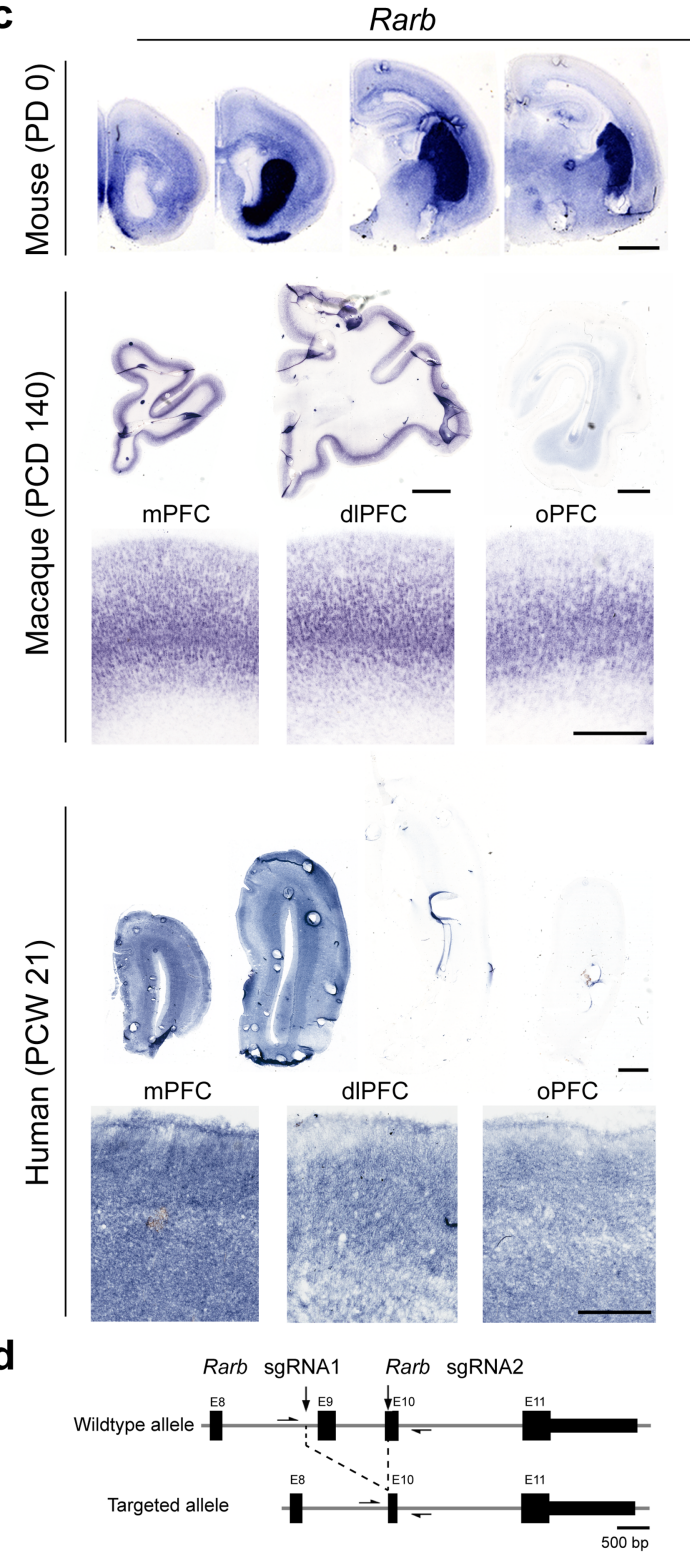

Extended Data Fig. $9 \mid$ RARB and RXRB are expressed in a moderate anterior-to-posterior gradient in the developing neocortex. a, Quantitative PCR analysis of Rar $a, b, g$ and $R x r a, b, g$ transcripts in the anterior and posterior half of mouse cortex at PD 0. Two-tailed Student'st-test: ${ }^{* * *} P=4 \mathrm{e}-4,1 \mathrm{e}-4 ; \mathrm{N}=3$ per condition; Errors bars: S.E.M.b, Quantitative PCR analysis of Rarb and Rxrg transcripts in four sections dissected out of the cortical plate in anterior-posterior direction. Both genes showed an anterior-posterior gradient in expression level. Two-tailed Student's $\mathrm{t}$-test; $\mathrm{N}=3$ per condition; b
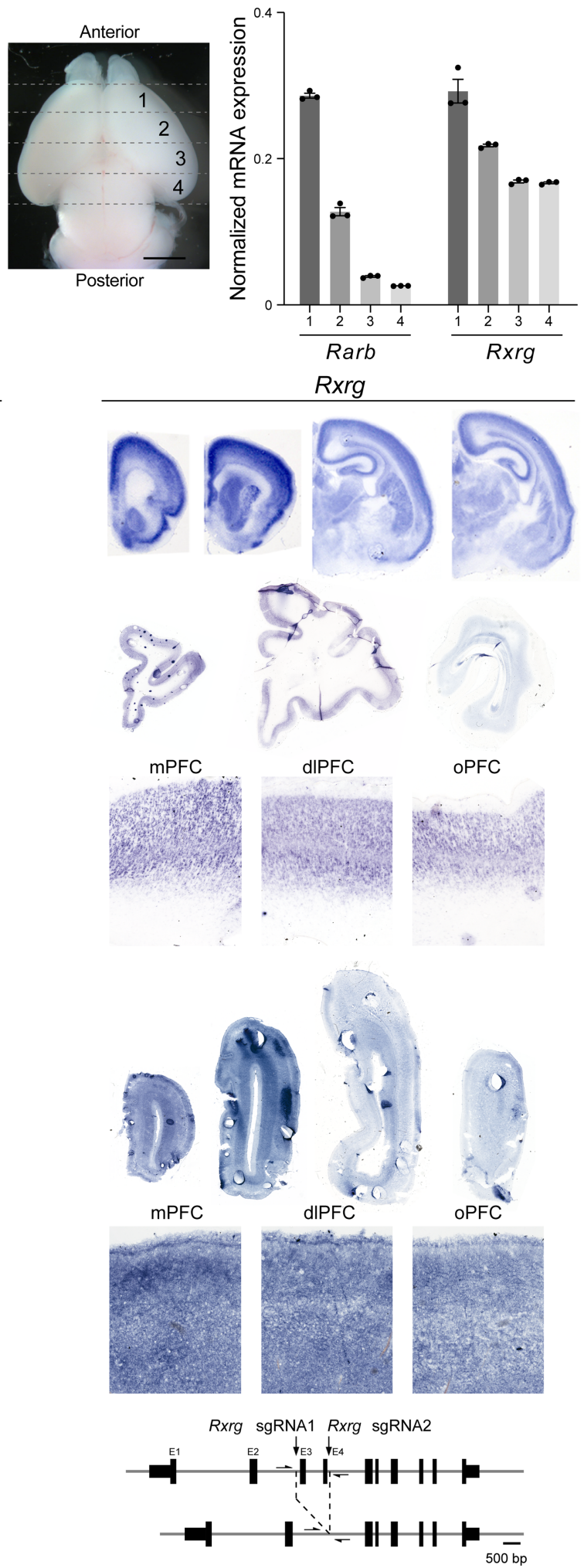

Errors bars: S.E.M.c, Expression of Rarb and Rxrg in mouse (PD 0), macaque (PCD 140) and human (PCW 21) brains by in situ hybridization. Higher magnification images of the regions of anterior cortex. Rarb and Rxrg transcripts are upregulated in the anterior part of the cortex in all three. Scale bars, $200 \mu \mathrm{m}$ (mouse); $2 \mathrm{~mm}$ (human); $500 \mu \mathrm{m}$ (human, higher magnification). $\mathrm{N}=2$ for human and macaque, $\mathrm{N}=3$ for mouse. $d$, Strategies for the generation of Rarb and Rxrg KO mice using CRISPR-Cas 9 technique ${ }^{41}$. 
a

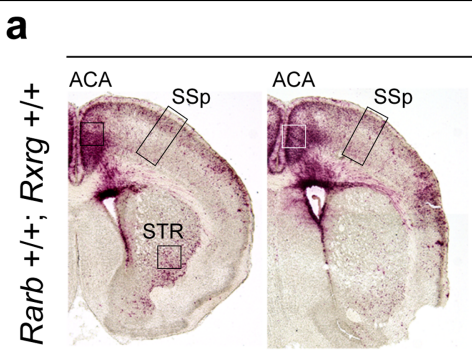

RARE-lacZ
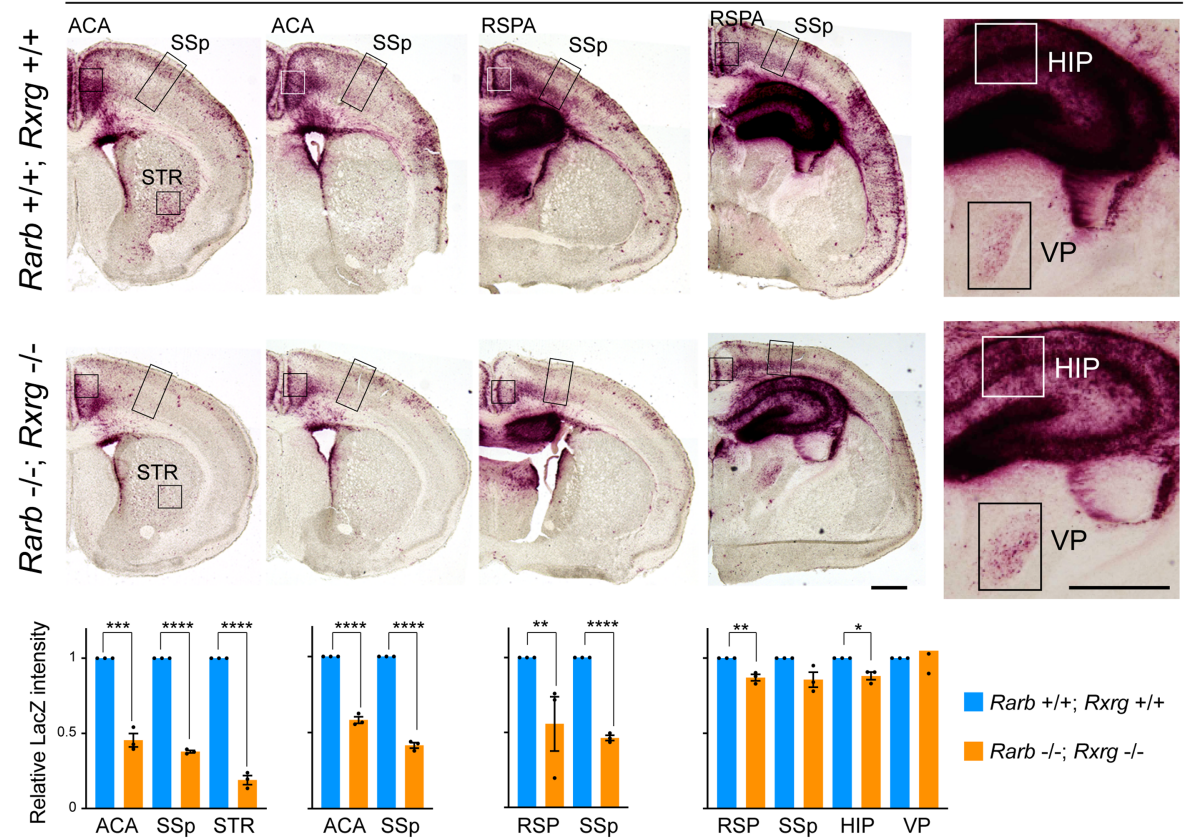

Rarb +/+; Rxrg +/+

Rarb -/-; Rxrg -/-

b

Cbln2 $\overline{\text { Rarb +/+; Rxrg +/+ Rarb -/-; Rxrg -/- }}$
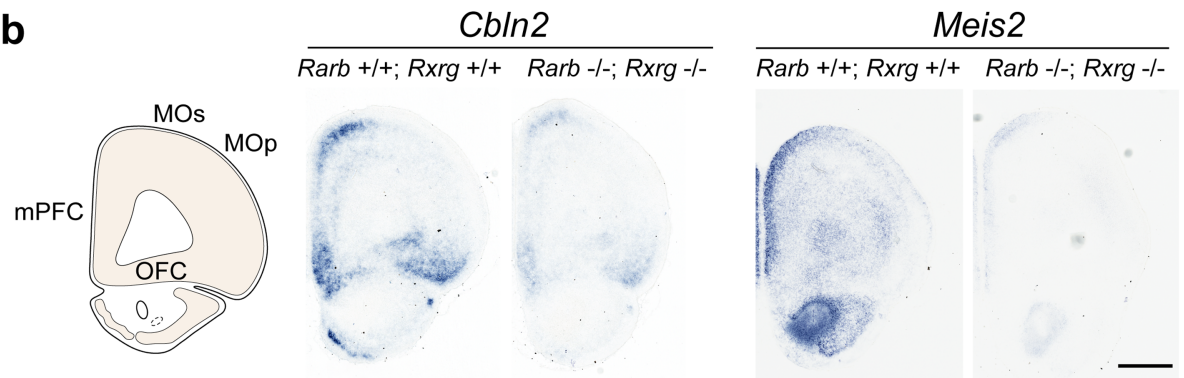

Extended Data Fig. 10 | RA signal in the neonatal mouse forebrain.

a, $\beta$-galactosidase histochemical staining of more posterior regions of $R a r b+/+$; Rxrg+/+(WT);RARE-lacZ and Rarb-/;Rxrg-/-(dKO);RARE-lacZ mouse brains at PD 0. Signal intensity in the boxed area (ACA, SSp, RSPA, STR, HIP and VP) was quantified. Note the reduced activity in anteromedial structures including ACA and RSPA (RSP). There is also reduced expression in HIP and lateral STR, but not the thalamus. Two-tailed Student'st-test; ${ }^{*} P=9 \mathrm{e}-3,{ }^{* *} P=7 \mathrm{e}-4 ; 3 \mathrm{e}-3$ (from left),
${ }^{* * *} P=2 \mathrm{e}-4,{ }^{* * * *} P=2 \mathrm{e}-7 ; 1 \mathrm{e}-5 ; 4 \mathrm{e}-5 ; 5 \mathrm{e}-6 ; 6 \mathrm{e}-6$ (from left). $\mathrm{N}=3$ per genotype: Errors bars: S.E.M.; Scale bars, $200 \mu \mathrm{m}$. b, Cbln 2 and Meis2 expression in PD 0 WT and dKO mutant brain by in situ hybridization at PD 0 . Note that $C b \ln 2$ and Meis2 expression in mPFC was decreased in dKO. Scale bar, $200 \mu \mathrm{m}$. N = 3 per genotype. ACA, Anterior cingulate area; CP, caudoputamen; HIP, Hippocampus; RSPA/RSP, Retrosplenial area;STR, striatum; VP, Ventroposterior thalamus. 
a

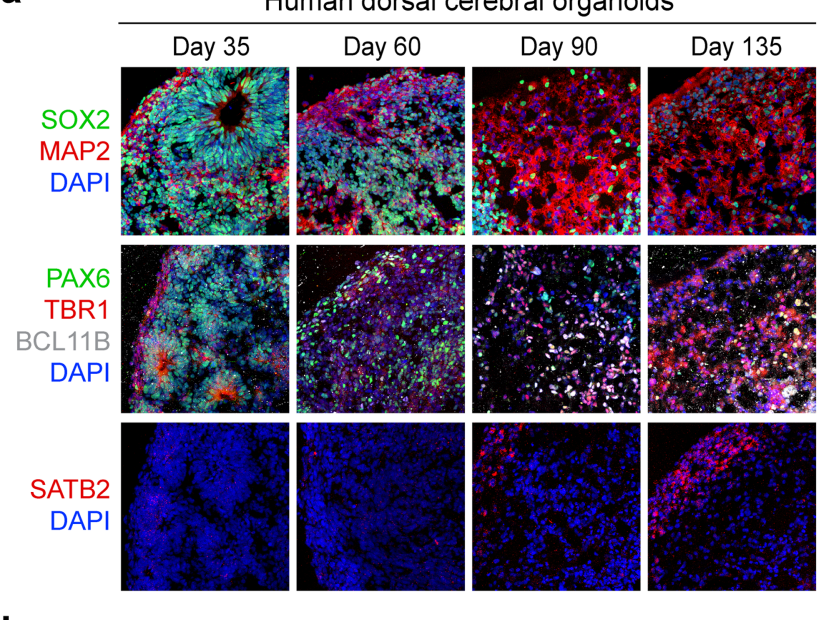

b

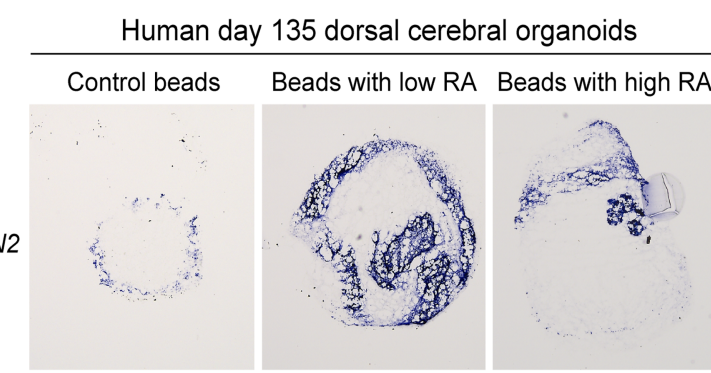

MEIS2
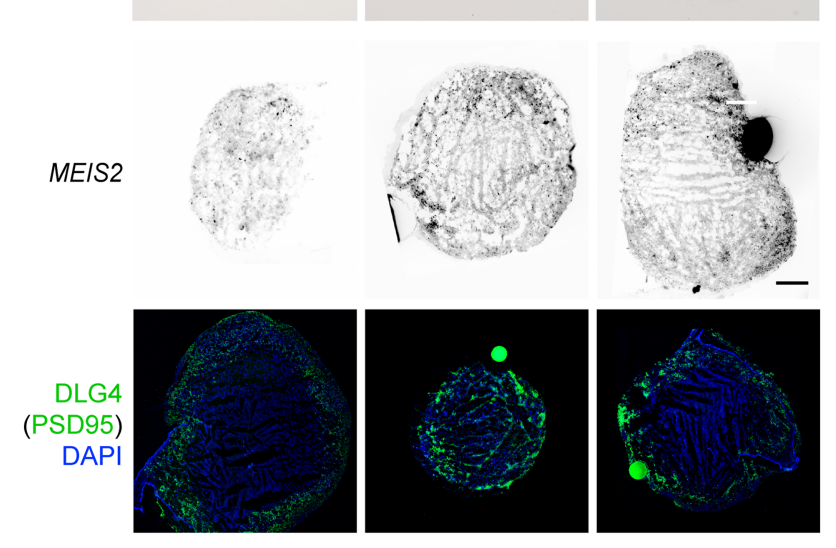

C
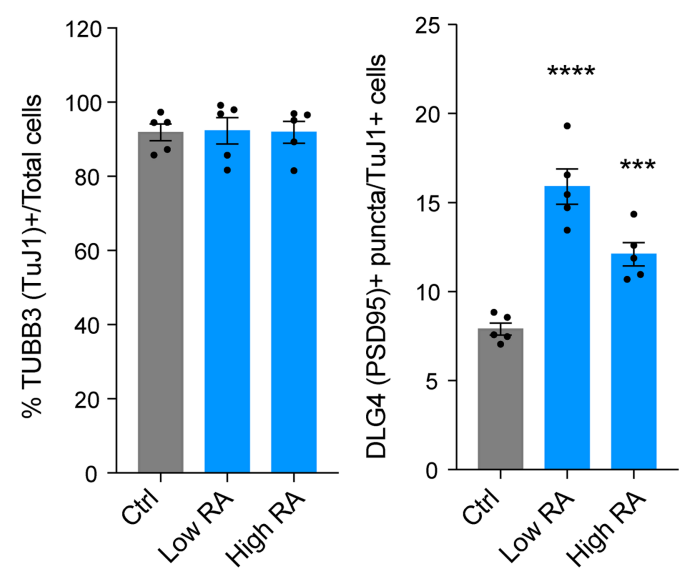

Chimpanzee dorsal cerebral organoids

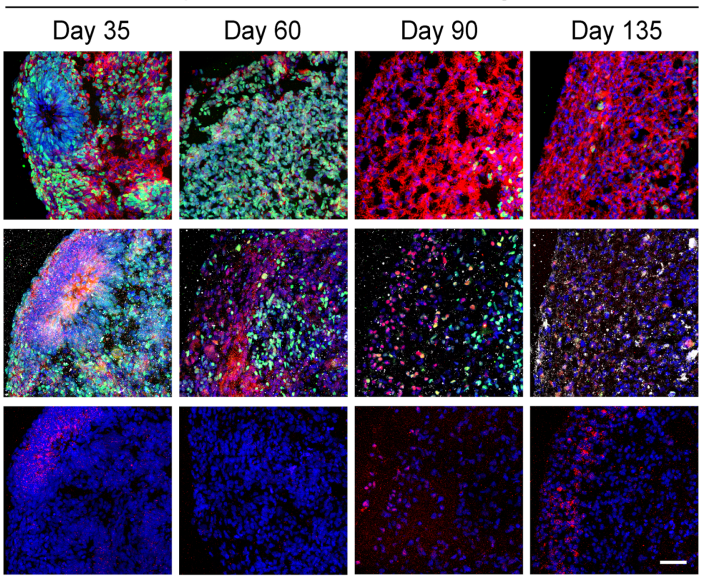

Chimpanzee day 135 dorsal cerebral organoids

Control beads Beads with low RA Beads with high RA
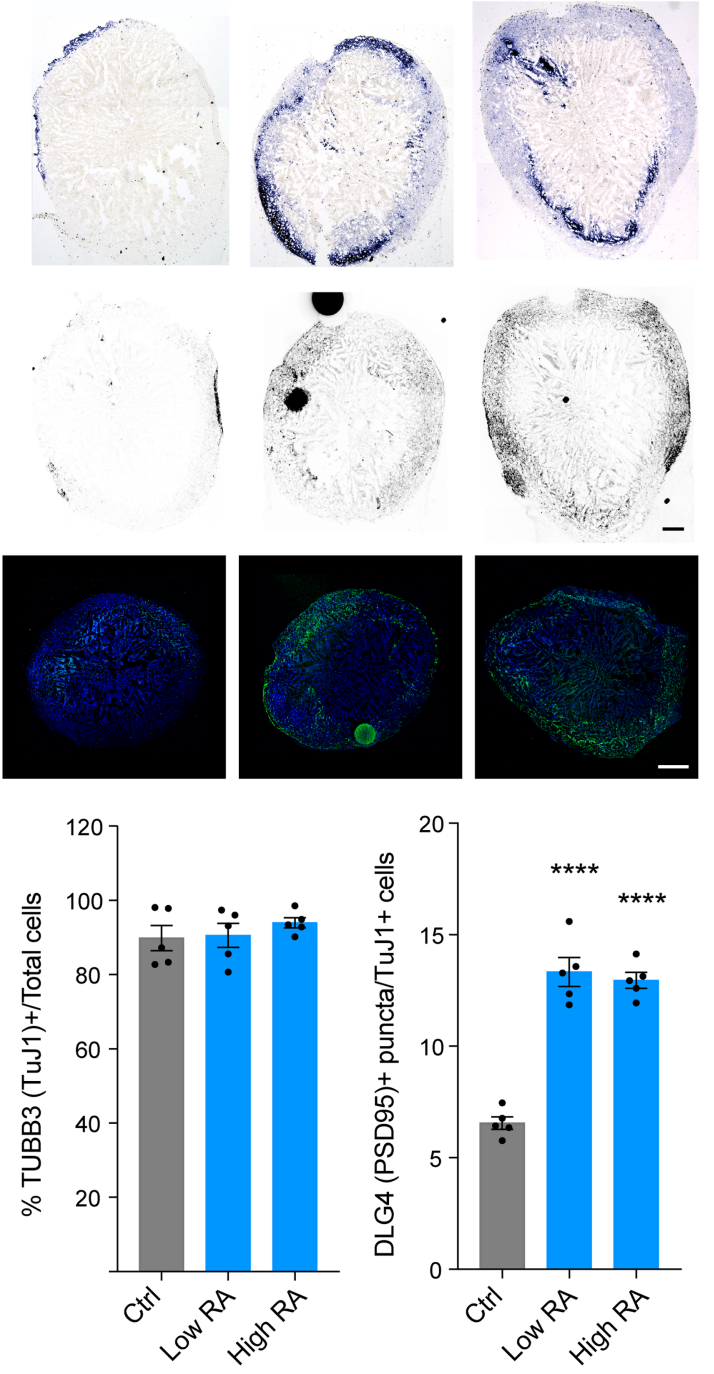

Extended Data Fig. 11 | See next page for caption. 


\section{Article}

Extended Data Fig. 11 | RA regulates CBLN2, MEIS2 and DLG4 (PSD95) expression in human and chimpanzee cerebral organoids. a, Expression of cortical neural stem/progenitor markers (PAX6 and SOX2), cortical cell typespecific markers (BCL11B, SATB2 and TBR1) and a pan-neuronal marker (MAP2) across the differentiation times show the dorsal cortical identity of the organoids derived from human and chimpanzee induced pluripotent stem cells. Scale bars, $50 \mu \mathrm{m}$. Each experiment used with 3-5 replicate organoids per times and conditions. b, In situ hybridization for CBLN2 and MEIS2,

immunostaining for PSD95/DLG4, and DAPI nucleic acid (nuclear) staining in human and chimpanzee day 135 organoids exposed to low or high concentration RA-soaked bead for $48 \mathrm{~h}$. Scale bars, $500 \mu \mathrm{m}$. One experiment has done with 3-5 replicate organoids per times and conditions. c, Proportion of TuJ1 immuno-positive neurons in day 135 cerebral organoids was similar across conditions. The ratio of total number of PSD95+ synaptic puncta to total number of TuJ1+ cells in the day 135 cerebral organoids was significantly increased in RA-soaked bead applied conditions. Two-tailed t-test, compared to the condition without bead (Control, $\mathrm{Ctrl}$ ), Errors bars: S.E.M. ${ }^{* * *} P=5 \mathrm{e}-4$, ${ }^{* * * *} P<1 \mathrm{e}-4 . \mathrm{N}=5$ (multiple sections for each organoid) per condition. 
a

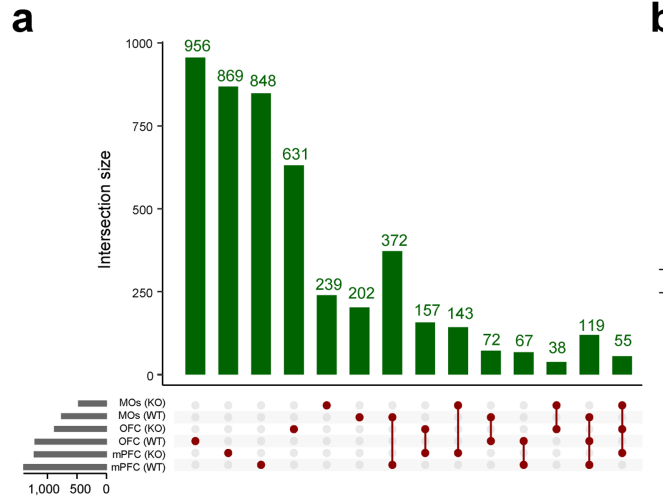

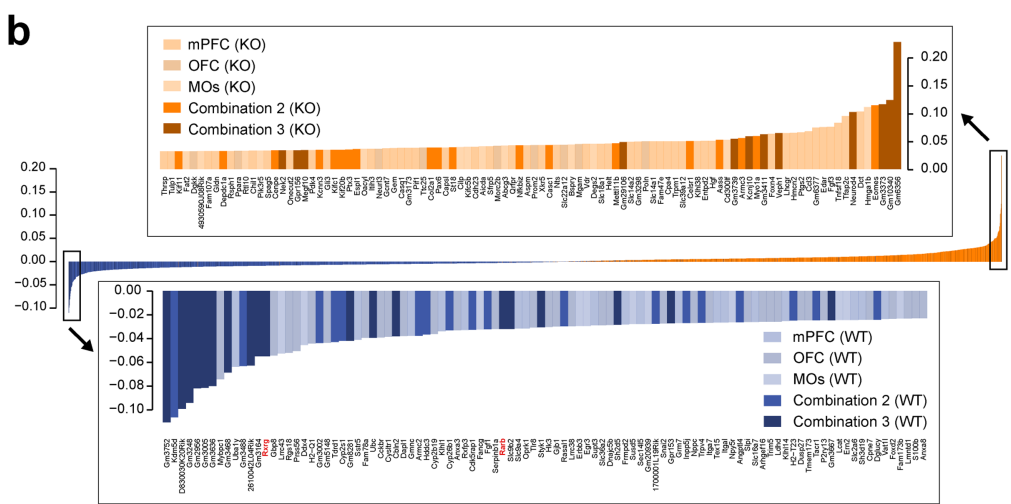

C
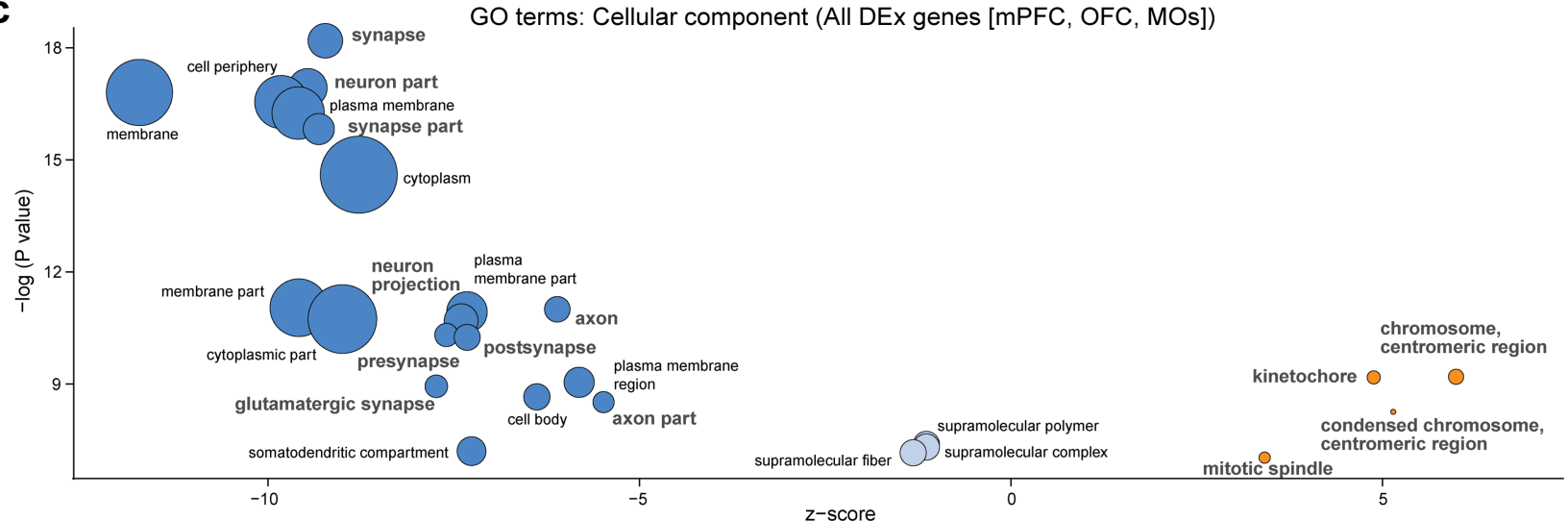

d

DEx genes shared in mPFC, OFC, MOs DEx genes only in mPFC

DEx genes only in OFC

DEx genes only in MOs
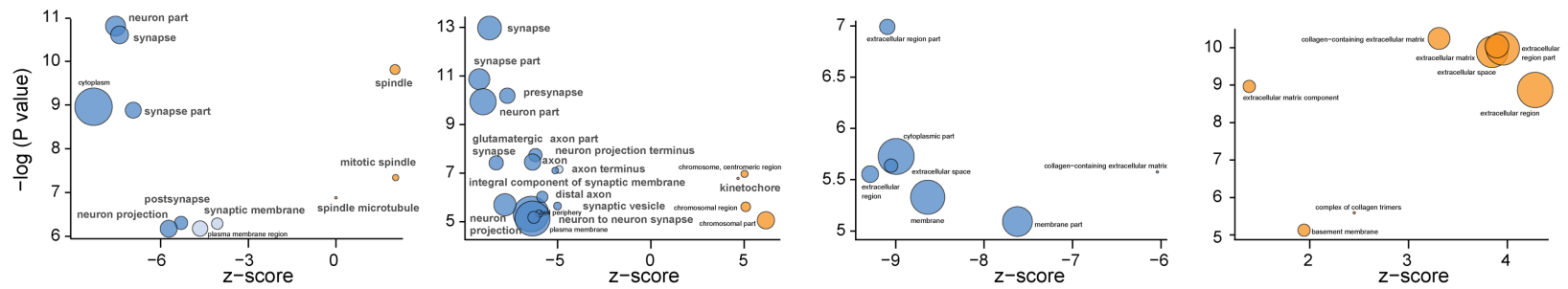

e
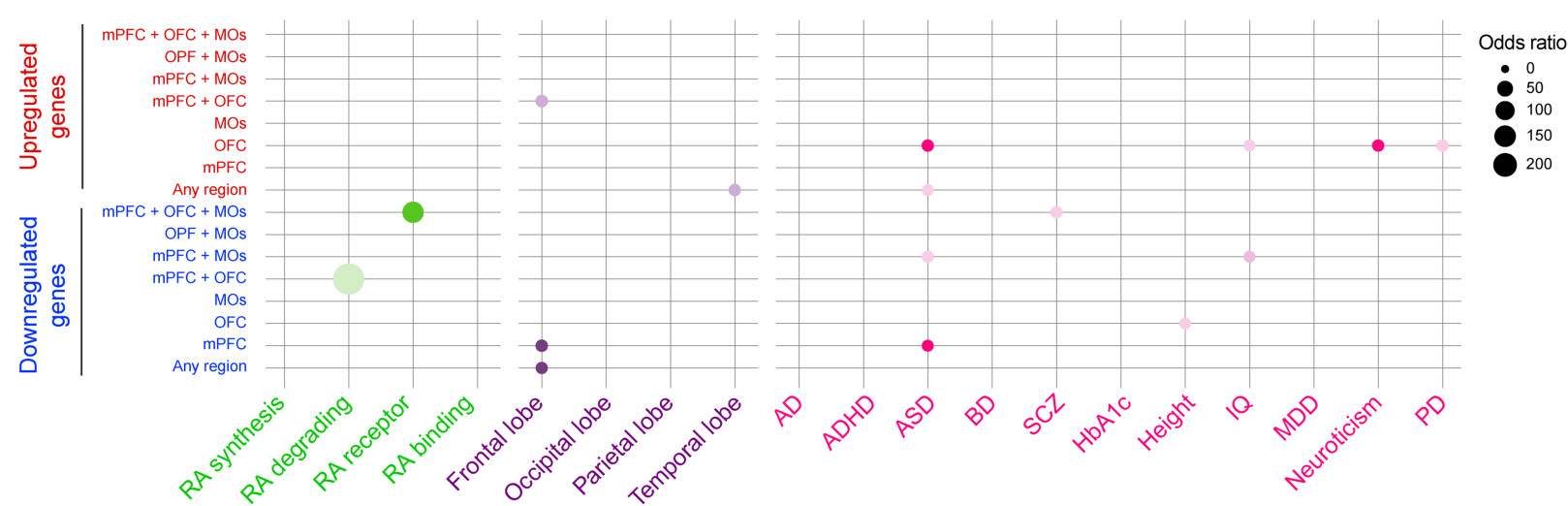

Extended Data Fig. 12 | Additional analysis of RNA-seq experiments.

a, Number of upregulated genes between PD O WT and dKO mice per region and phenotype, as well as combinations of regions and phenotypes. b, Gene loadings of the first principal component from PCA in Fig. 3. Colours represent the frontal cortex region where the gene was found to be upregulated. c, Cellular component $\mathrm{GO}$ terms associated with the total list of 4,768 DEx genes found, showing their Z-score and nominal $P$ values. Z-score represents the proportion of upregulated versus downregulated genes in the list of DEx genes associated to each GO term (i.e. z-score $=(\#$ up - \#down $) /$ sqrt $(\#$ all DEx associated to the GO term)). Dark blue: $z$-score $<-5$; light blue: $z$-score $(-5,0]$; orange: $z$-score $>0$. Size of the bubbles are proportional to the total number of

DEx genes associated to the given GO term. d, Cellular component GO terms associated with DEx genes found in all three frontal cortex regions, and DEx genes unique to each region ( $\mathrm{mPFC}, \mathrm{OFC}, \mathrm{MOs}$ ) and their unadjusted $\mathrm{p}$ value. e, Enrichment of RA related genes (green), genes upregulated in individual lobes of the mid-fetal human cortex based on Fig. 1 (purple), and psychiatric disease related genes in up- and downregulated genes (pink). DEx genes are separated by genes that are DEx only in the given region ( $\mathrm{MPFC}, \mathrm{OFC}, \mathrm{MOs}$ ), genes that are DEx in the two given regions ( $\mathrm{mPFC}+\mathrm{OFC}, \mathrm{mPFC}+\mathrm{MOs}, \mathrm{OFC}+\mathrm{MOs})$, genes that are DEx in all three regions ( $\mathrm{mPFC}+\mathrm{OFC}+\mathrm{MOs}$ ). Circles plotted for significant enrichments ( $P$ value $<0.05$ ), in darker colour, significance is considering the adjusted $\mathrm{p}$ value. Diameter of circle is associated with odds ratio per legend. 
a
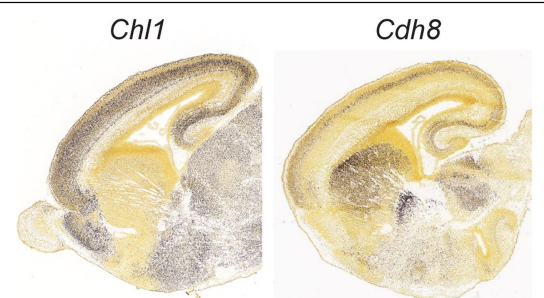

$N t f 3$
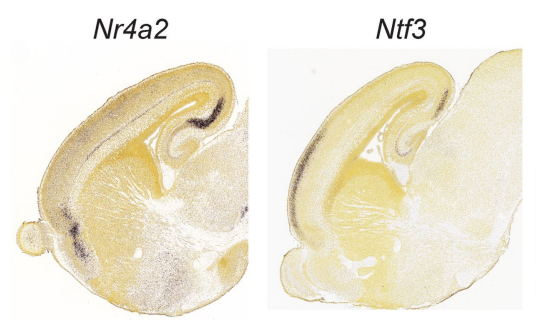

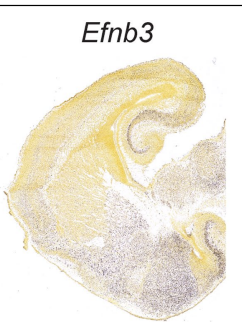

Plxnc1

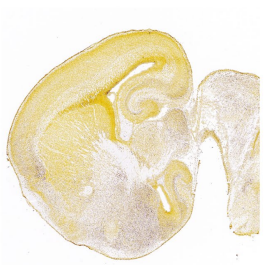

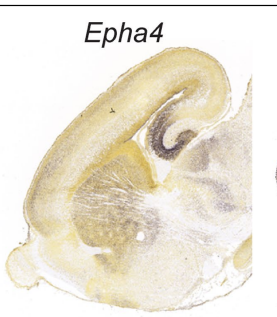

Plxnd1

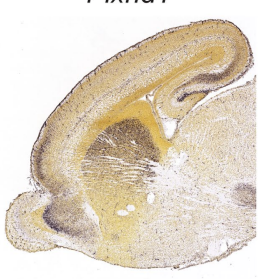

Epha8
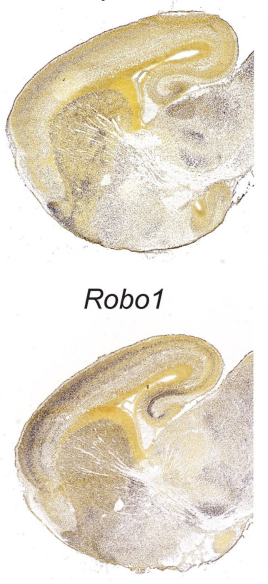

Ephb6
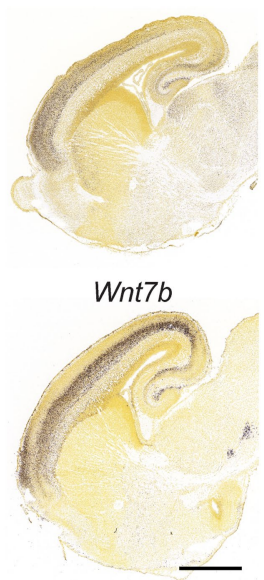

b

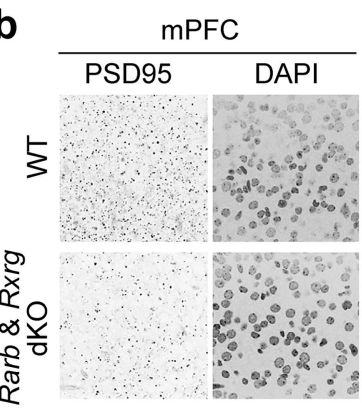

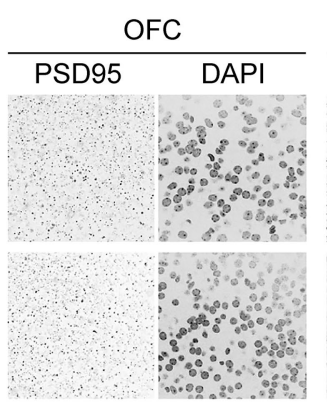

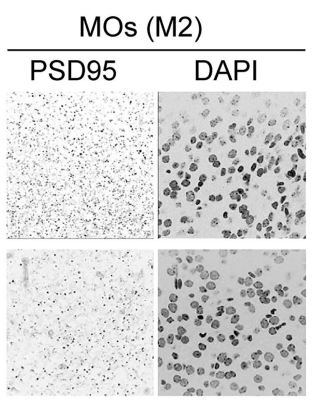

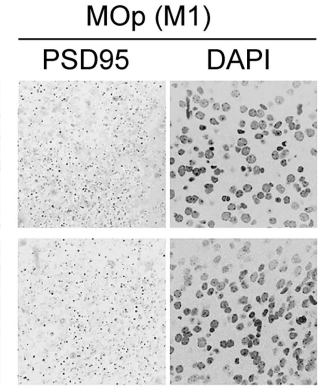

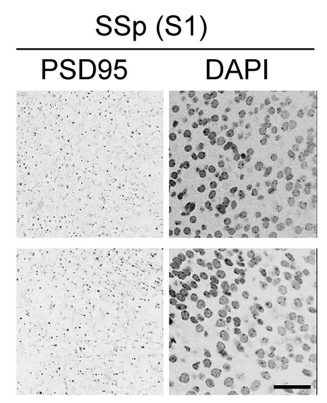

C

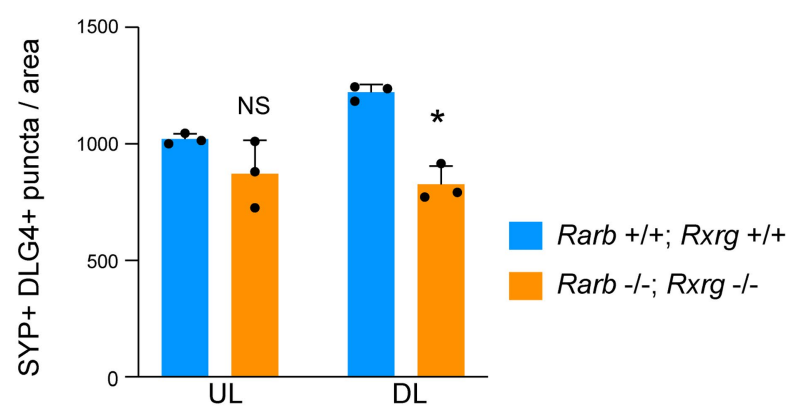

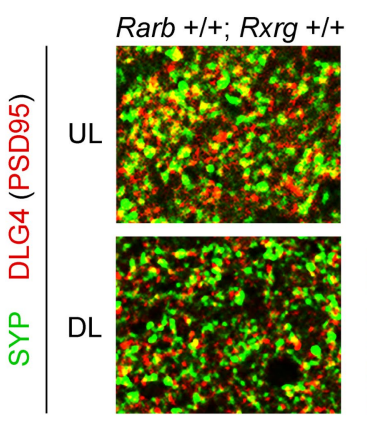

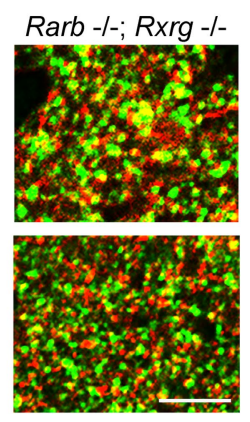

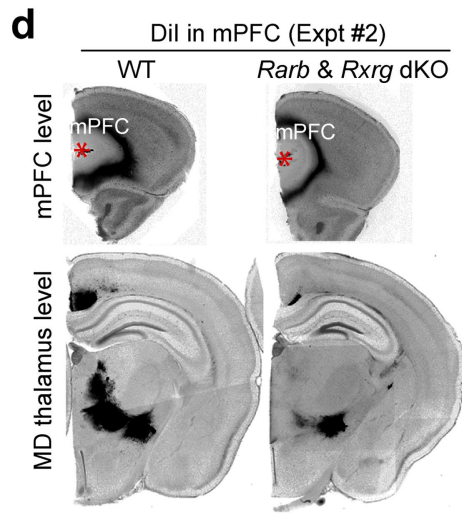
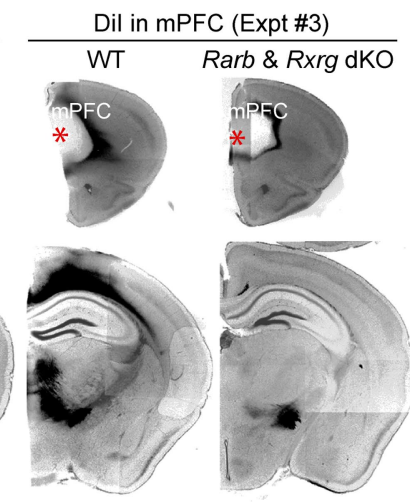

Extended Data Fig. 13 | Altered synaptic density and axonal projections in Rxrg and Rarb dKO mice. a, Example of downregulated genes between PD 0 Rarb $+/+; R x r g+/+(\mathrm{WT})$ and $R a r b-/-; R x r g-/-(\mathrm{dKO})$ that displayed an anterior to posterior gradient in PCD 18 mouse embryo (images are from the Allen Developing Mouse Brain Atlas; developingmouse.brain-map.org $\left./{ }^{58}\right)$. Scale bar: $1 \mathrm{~mm} . \mathbf{b}$, Immunostaining for PSD95/DLG4 in the cortical subregions (mPFC, MOs, OFC, MOp, and SSp) of WT and dKO brain at PD 0. Each region as shown in

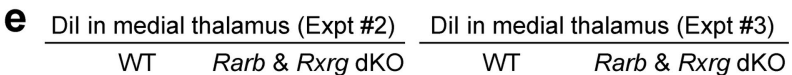
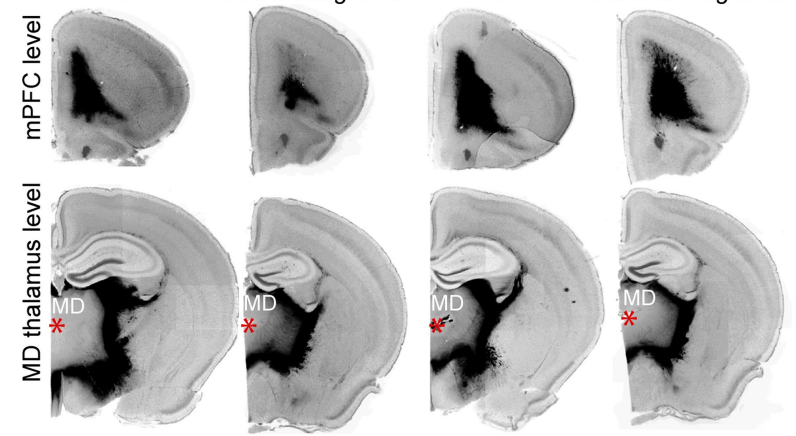

Fig. 2c. Scale bar: $25 \mu \mathrm{m} . \mathrm{N}=3$ per genotype. $\mathrm{c}$, Quantification and representative images of co-localized synaptophysin (SYP) and PSD95 puncta in PD 0 WT and dKO mPFC. Two-tailed Student'st-test; ${ }^{*} P=0.01 ; \mathrm{N}=3$ per genotype; Errors bars: S.E.M.;Scale bar: $10 \mu \mathrm{m}$. d, e, Dil placement in mPFC (c) and medial thalamus (d) with tracing data in WT and dKO brain at P21. Additional two replicates of experiment shown in Fig. 4d, e are shown. Asterisks: Dil crystal placement. Scale bar: $1 \mathrm{~mm}$. 


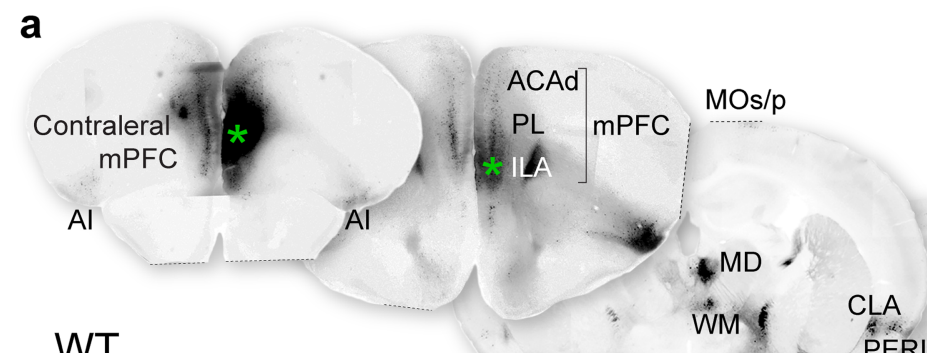

WT

$($ Rarb +/+; Rxrg +/+)

b

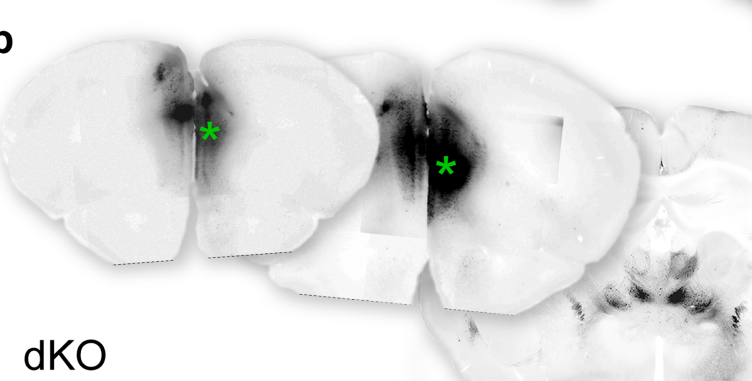

(Rarb -/-; Rxrg -/-)

C
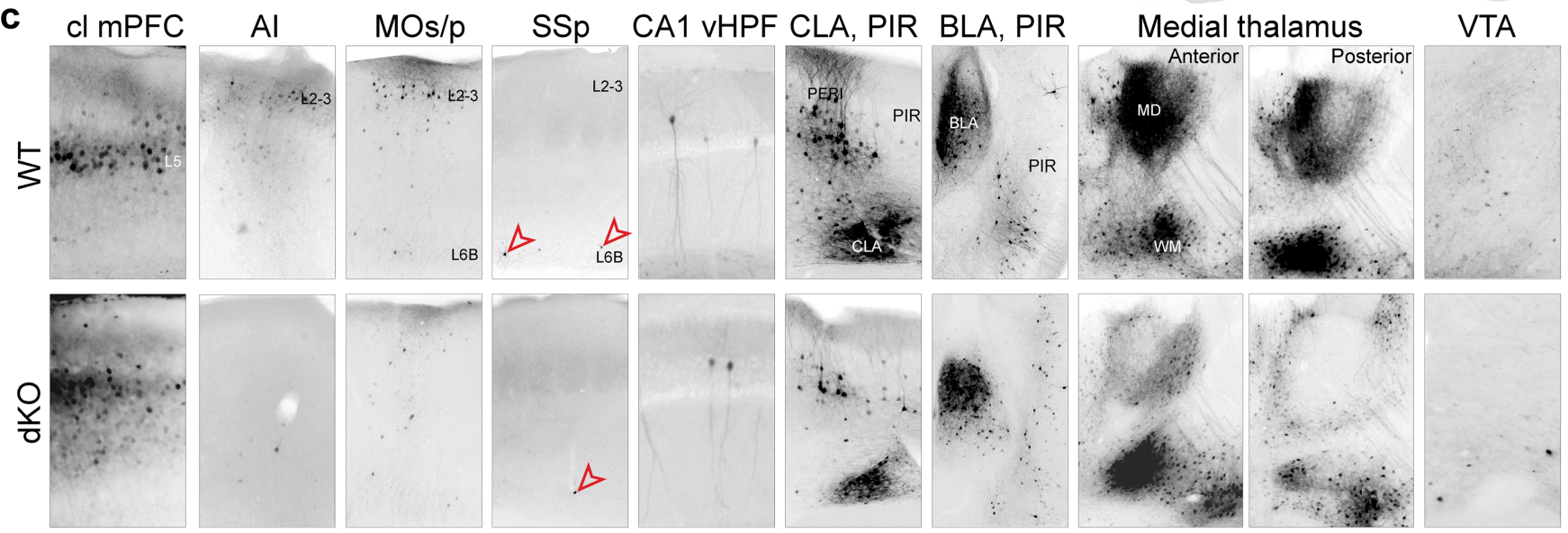

d
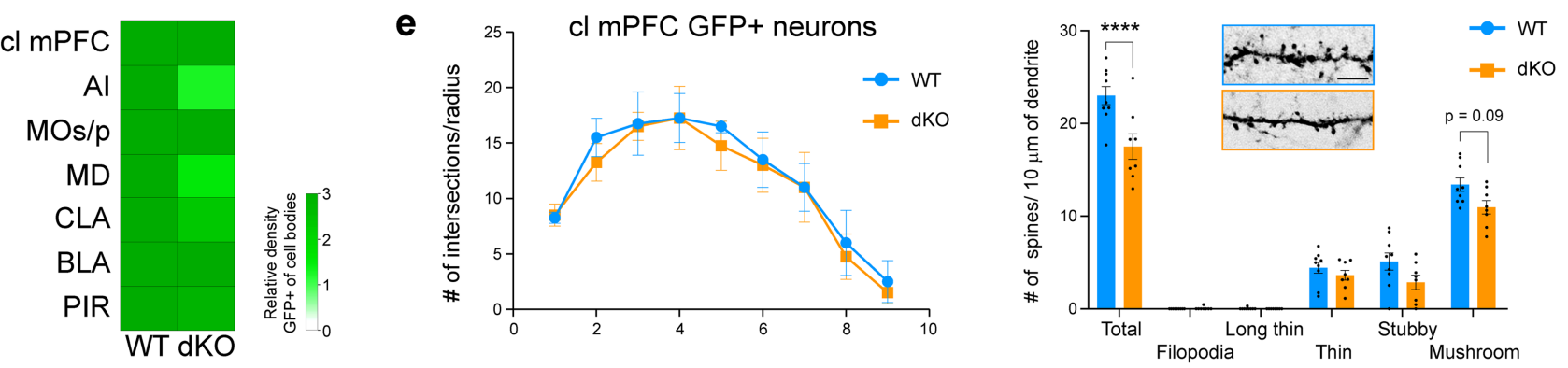

Extended Data Fig. 14 | See next page for caption. 


\section{Article}

Extended Data Fig. $14 \mid$ Analysis of axonal inputs to the mPFC and dendritic spines in Rarb and Rxrg dKO mice. Anteroposterior series of PD 30 coronal sections of representative WT (a) and $\mathrm{dKO}(\mathbf{b})$ with retrograde viral tracer AAVrg-Cag-Gfp injected into mPFC (green asterisk). Sections were inverted and converted to greyscale. Black regions indicate the presence of viral tracer. High magnification representative images of labelled regions (c) and quantification of labelling using 0-3 scale (d) $(\mathrm{N}=4$ for each genotype; see Methods section for more details). e, Quantification of Sholl analysis (Two-way ANOVA with Sidak's multiple comparison method; $P=.440->0.999)$; Error bars: S.E.M.) and dendritic spines of upper layer neurons in the contralateral mPFC in WT (blue) and dKO (orange) $(\mathrm{N}=4$ for each genotype). Two-way ANOVA with Sidak's multiple comparison method was applied; ${ }^{*} P=0.09$; ${ }^{* * * *} P<1 \mathrm{e}-4$; Error bars: S.E.M. Inset shows representative images of dendritic spines. Scale bar: $5 \mu \mathrm{m}$. ACAd, Dorsal anterior cingulate area; AI, Anterior Insula; BLA, Basolateral amygdala; vHPF, ventral hippocampal fields; CLA, claustrum; cl mPFC, Contralateral medial prefrontal cortex; ILA, Infralimbic area; LHA, Lateral habenula; MD, The mediodorsal nucleus of the thalamus; MOs/p, The secondary and primary motor areas. PER, Perirhinal cortex PIR, Piriform cortex PL, Prelimbic area; SSp, Primary somatosensory area; STN, Subthalamic nucleus; VTA, Ventral tegmental area. 


\begin{tabular}{c|c|c|c|} 
Human fetal neocortical progenitors and neurons & Differentiation and maturation \\
PCW 8 & Expansion and passage & Day 14 & -/+RA or RARi (AGN193109)
\end{tabular}
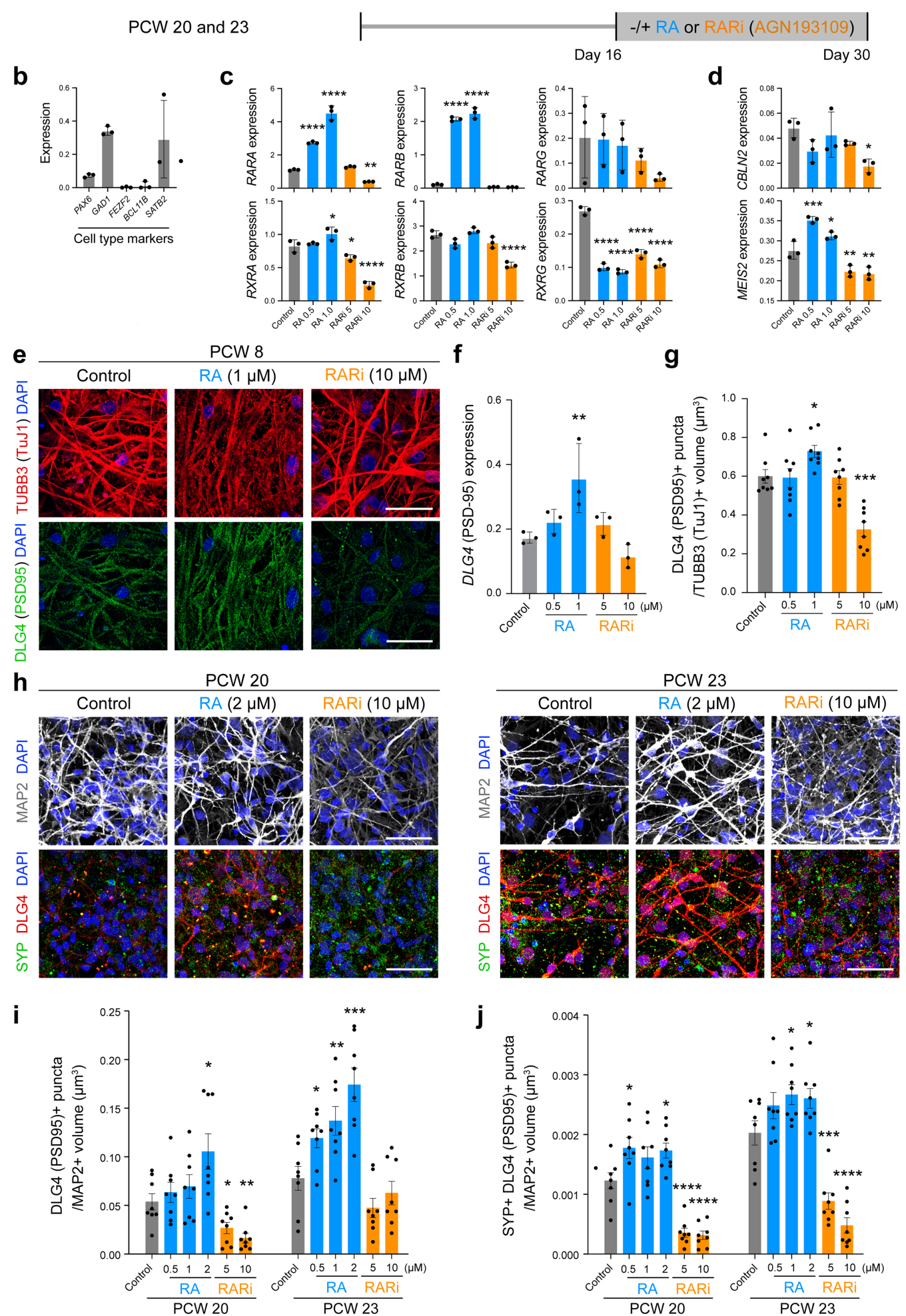

Extended Data Fig. 15 |See next page for caption.

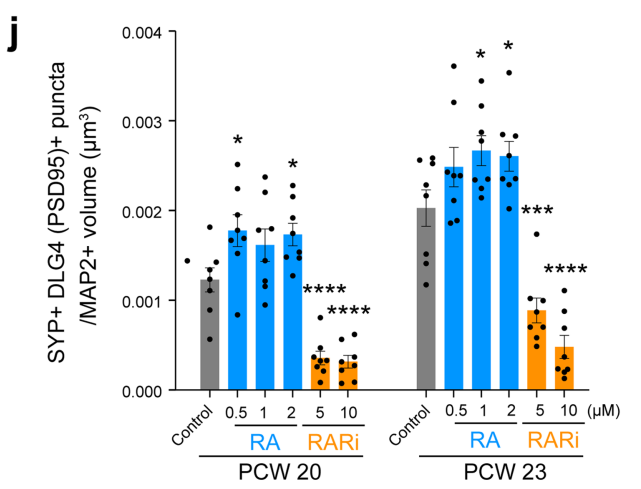




\section{Article}

Extended Data Fig. 15 | RA regulates CBLN2, MEIS2, and DLG4 (PSD95) expression and synaptogenesis in human fetal neocortical neurons.

a, Schematics of human cortical neuron differentiation and modulation of RA signalling. From PCW 8 neocortical tissue, cortical neural stem cells were isolated and expanded with FGF2 for 20 days. Neural differentiation was initiated by the mitogen withdrawal. After 14 days of differentiation, cells were treated with varying doses of RA or RA receptor inhibitor (RARi, AGN193109) for another 14 days. From PCW 20 or 23 neocortical tissue, cortical neural progenitors and neurons were isolated and cultured without FGF2. After 16 days of culture, cells were treated with RA or RARi for another 14 days. Gene expression of cell type markers (b), RA receptors (c) RA regulated genes CBLN2 and MEIS2 (d) in PCW 8 cortical cells after 28 days of differentiation, measured by ddPCR. One way ANOVA and multiple comparison, compared to vehicle (0.01\% DMSO)-treated control. Errors bars: S.E.M. ${ }^{*} P<0.05(R X R A$ expression, RA1.0, $P=0.0192$, RARi5, $P=0.0277 ; C B L N 2$ expression, RARi10, $P=0.0135$ MEIS2 expression, RA1.0, $P=0.0456),{ }^{* *} P<0.01(R A R A$ expression, RARi10, $P=0.0039 ;$ MEIS 2 expression, RARi5, $P=0.0057$, PARi10, $P=0.0028)$,

${ }^{* * *} P<0.001$ (MEIS2 expression, RA0.5, $P=0.0003$ ), ${ }^{* * * *} P<0.0001 . \mathrm{N}=3$ experimental replicates per condition. e, TUBB3/TUJ1 and DLG4/PSD95 expression in RA or RARi-treated PCW 8 cortical cells. Scale bar: $50 \mu \mathrm{m}$. f, DLG4/PSD95 transcript in PCW 8 cortical cells was significantly increased in RA $1 \mu \mathrm{M}$ condition quantified by ddPCR. Two-tailed t-test, compared to the vehicle treatment. Errors bars:S.E.M. ${ }^{* *} P=0.0083 . \mathrm{N}=8$ fields per condition. g, The ratio of total DLG4/PSD95+ puncta volume to total TUJ1+ volume in PCW 8 cortical cells was increased in the RA condition and reduced in the RARi condition. Two-tailed t-test, compared to the vehicle treatment. Errors bars: S.E.M. ${ }^{*} P=0.0152,{ }^{* * *} P=0.0001$. h, MAP2, synaptophysin (SYP) and DLG4/ PSD95 expression in RA or RARi-treated PCW 20 and 23 cortical cells. Scale bar: $50 \mu \mathrm{m}$. $\mathbf{i}$, The ratio of total DLG4/PSD95+ puncta volume to total MAP2+ volume in PCW 20 and 23 cortical cells was increased in RA conditions and reduced in RARi conditions. Two-tailed t-test, compared to the vehicle treatment. Errors bars: S.E.M. ${ }^{*} P<0.05$ (PCW 20-RA2, $P=0.0221$; PCW 20 -RARi5, $P=0.0193$; PCW 23 -RA0.5, $P=0.0192$ ), ${ }^{* *} P<0.01$ (PCW 20-RARi10, $P=0.0018$; PCW 23-RA1, $P=0.0081),{ }^{* * *} P=0.0004 . \mathrm{N}=8$ fields per condition. $\mathbf{j}$, The ratio of total number of SYP+ and DLG4/PSD95+ colocalized synaptic puncta to total MAP2+ volume in PCW 20 and 23 neocortical cells was increased in RA conditions and reduced in RARi conditions. Two-tailed t-test, compared to the vehicle treatment. Errors bars: S.E.M. ${ }^{*} P(\mathrm{PCW} 20$-RA0.5, $P=0.0271$; PCW 20-RA2, $P=0.0159$; PCW 23$\mathrm{RA} 1, P=0.0289 ; \mathrm{PCW} 23-\mathrm{RA} 2, P=0.0447),{ }^{* * *} P=0.0004,{ }^{* * * *} P<0.0001 . \mathrm{N}=8$ fields per condition. 

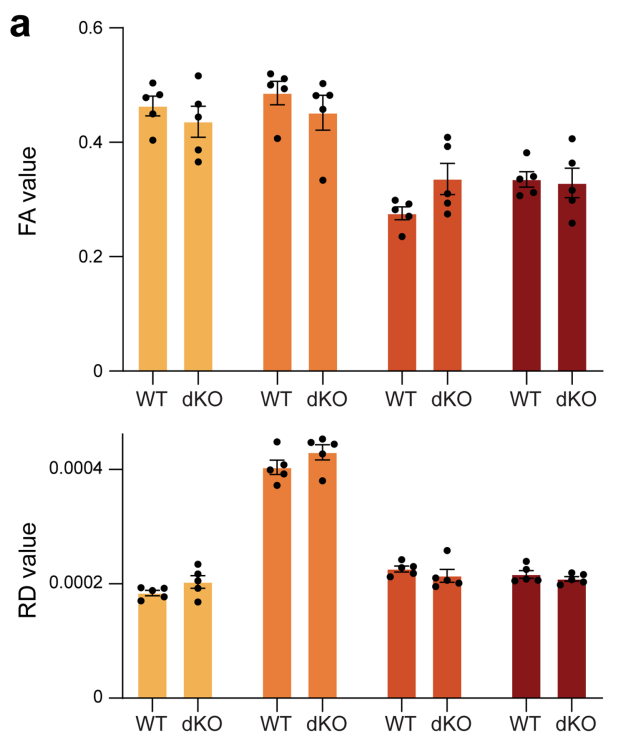

b

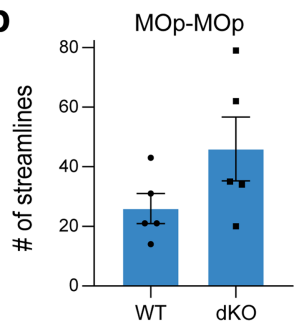

C

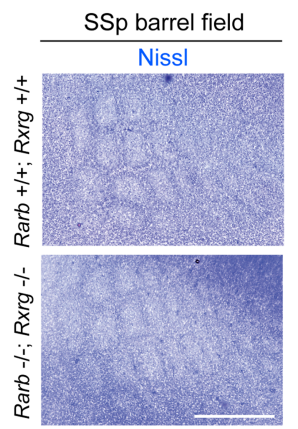

d
Corpus callosum

- Anterior commissure

- Internal capsule - Left

- Internal capsule - Right
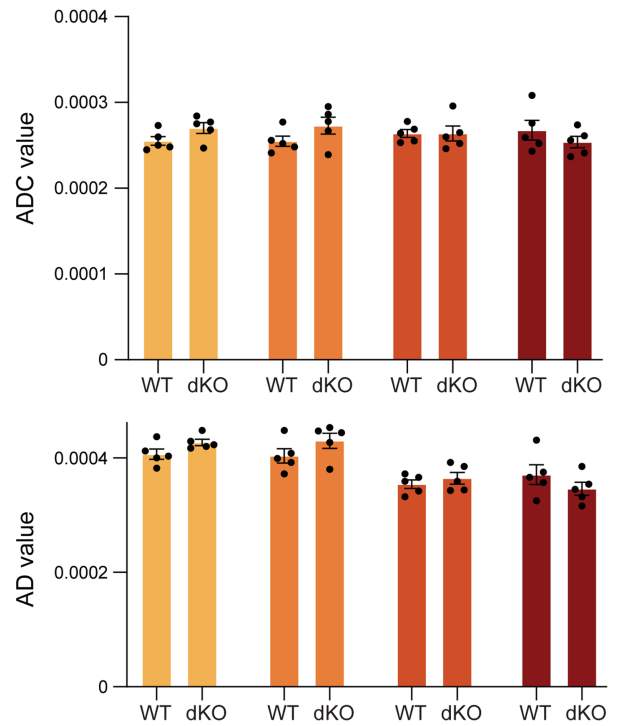

AUDp-Th

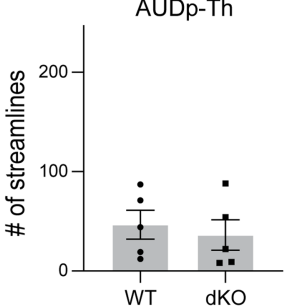

ACA-Th

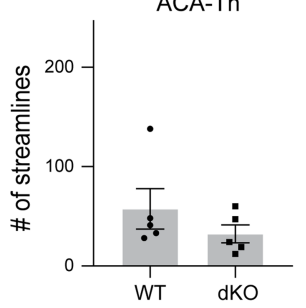

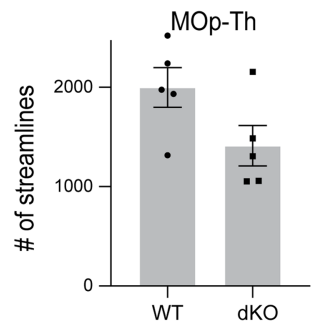
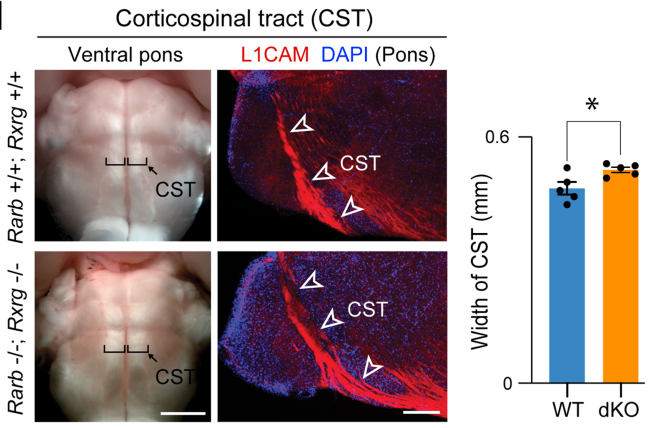

e Cell death in mPFC

f

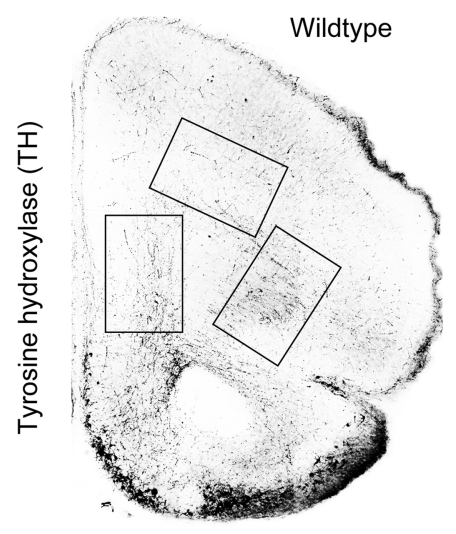

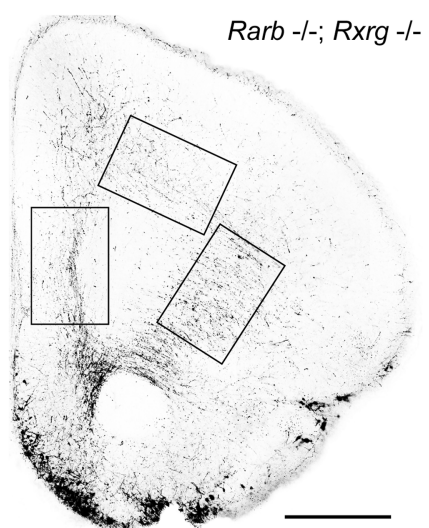

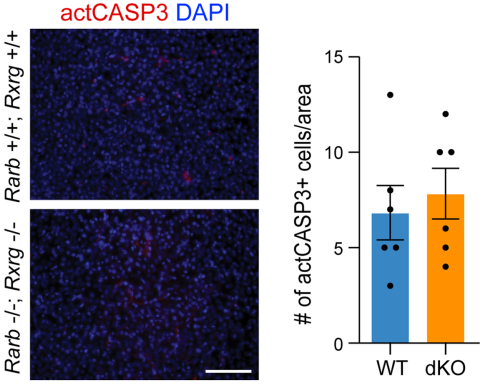

Extended Data Fig. 16 | See next page for caption.

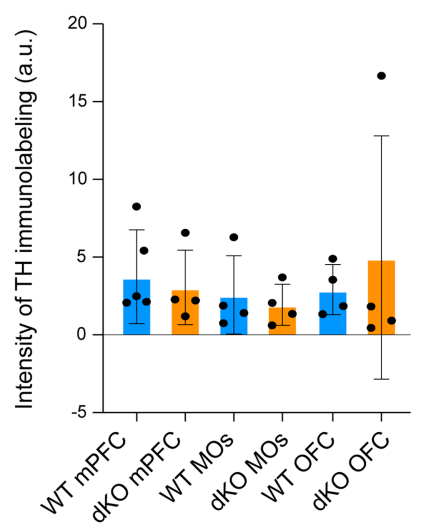




\section{Article}

Extended Data Fig. 16 | Analysis of axonal projections and cell death in Rarb and Rxrg dKO mice. a, Four scalar indexes which describe microstructural integrity do not differ in the four major white-matter tracts (corpus callosum, anterior commissure, left and right internal capsules) between WT and dKO mice. FA Comparisons: Corpus callosum (two-tailed unpaired t-test, $P=0.4$ ); Anterior cingulate (two tailed mann-whitney test, $P=0.2$ ); Internal Capsule left (two-tailed unpaired t-test, $P=0.07$ ); Internal Capsule right (two-tailed unpaired t test, $P=0.8$ ). ADC Comparisons: Corpus callosum (two-tailed unpaired t-test, $p$ value 0.1); Anterior cingulate (two-tailed unpaired t-test, $p$ value 0.1); Internal Capsule left (two-tailed unpaired t-test, $p$ value 0.9); Internal Capsule right (two-tailed unpaired t-test, $P=0.3$ ). RD Comparisons: Corpus callosum (two-tailed unpaired t-test, $P=0.1$ ); Anterior cingulate (two-tailed unpaired t-test, $P=0.1$ ); Internal Capsule left (two tailed mannwhitney test, $P=0.1$ ); Internal Capsule right (two-tailed unpaired t-test, $P=0.3$ ). AD Comparisons: Corpus callosum (two-tailed unpaired t-test, $P=0.08$ ); Anterior cingulate (two-tailed unpaired t-test, $P=0.1$ ); Internal Capsule left (two-tailed unpaired t-test, $P=0.4$ ); Internal Capsule right (two-tailed unpaired t-test, $P=0.2$ ). N=5; Errors bars: S.E.M. b, Number of streamlines of MOpthalamus and corticocortical tracts, did not differ between WT and dKO. Twotailed unpaired t-test for MOp-MOp, MOp-Th, and AUDp-th ( $P=0.1,0.07$, and 0.6 , respectively). Two-tailed Mann-Whitney test for ACA-Th $(P=0.3) . \mathrm{N}=5$ per genotype; Errors bars: S.E.M.c, Barrel formation was examined by Nissl staining at PD 5 in $\mathrm{WT}$ and $\mathrm{dKO} . \mathrm{N}=3$ per genotype. $\mathbf{d}$, Representative images and quantification of corticospinal tract (CST) width using L1CAM expression at PD 5 in WT and dKO. The width of the corticospinal tract (shown in brackets) is slightly increased in dKO. Two-tailed Student's t-test: ${ }^{*} P=0.02 ; \mathrm{N}=5$ per genotype; Errors bars: S.E.M.e, Representative images and quantification of of apoptotic cells in the MPFC detected by cleaved caspase 3 (actCASP3) between WT and dKO. Two-tailed Student'st-test: WT vs. dKO; NS; $\mathrm{N}=5$ per genotype; Errors bars:S.E.M.;Scale bars: $500 \mu \mathrm{m} ; 200 \mu \mathrm{m}$ (b,f); $1 \mathrm{~mm}$ (c); $100 \mu \mathrm{m}$ (d). f, Representative images and quantification of tyrosine hydroxylase $(\mathrm{TH})$ immunolabelled axons in WT and dKO mouse frontal cortex at PD $5(\mathrm{~N}=4)$. Rectangles represent regions analysed. Clockwise starting from left-most: mPFC, MOs, OFC. Errors bars: S.E.M. 
a
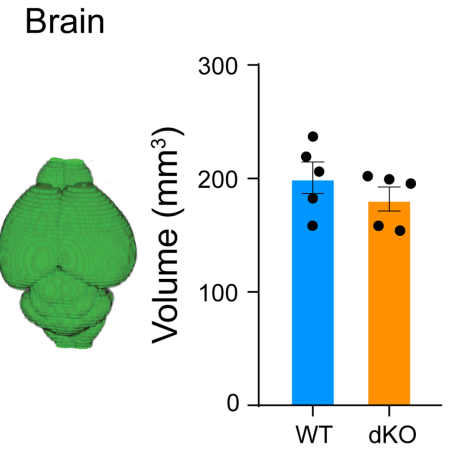

b

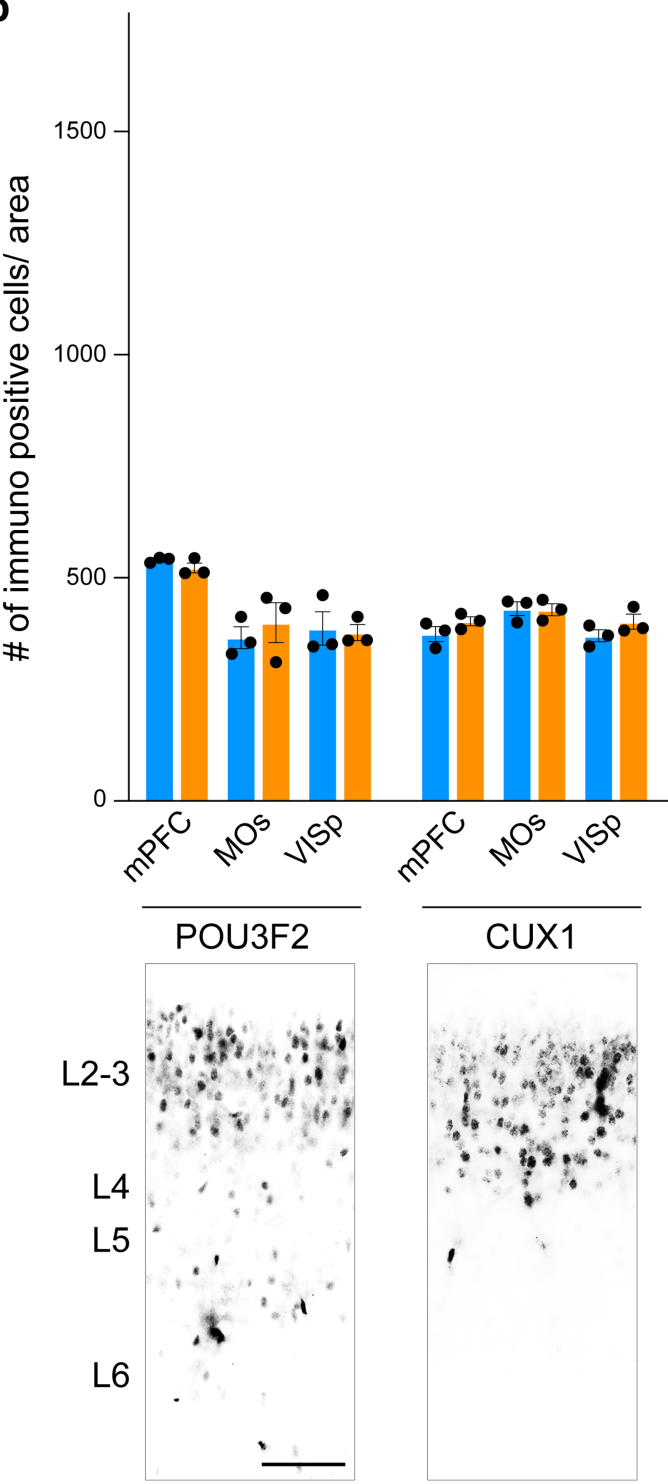

Extended Data Fig. 17|Analysis of neocortical layers and tissue volume in Rarb and Rxrg dKO mice. a, Representative images and quantification of total volume of the brain (left), cerebral neocortex (middle), anterior one-third of the neocortex (right) of WT littermates and Rarb;Rxrg dKO at PD 5. Two-tailed unpaired $t$-test for total volume of the brain and anterior one-third of the neocortex. Two-tailed Mann-Whitney test for volume of cerebral neocortex.
Anterior $1 / 3$ of the neocortex
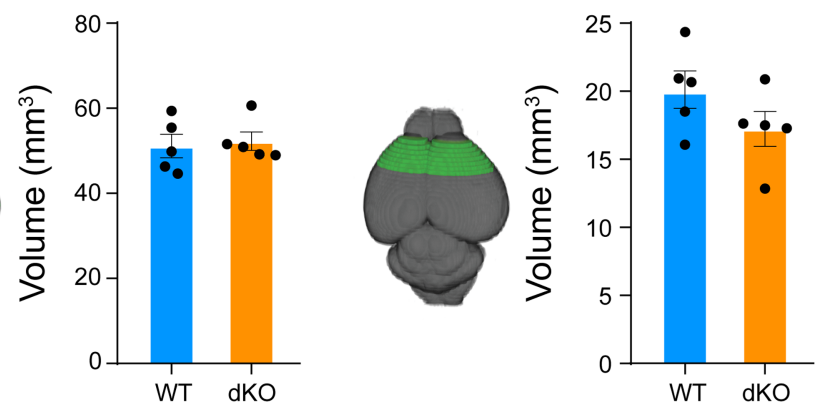

$\bullet$

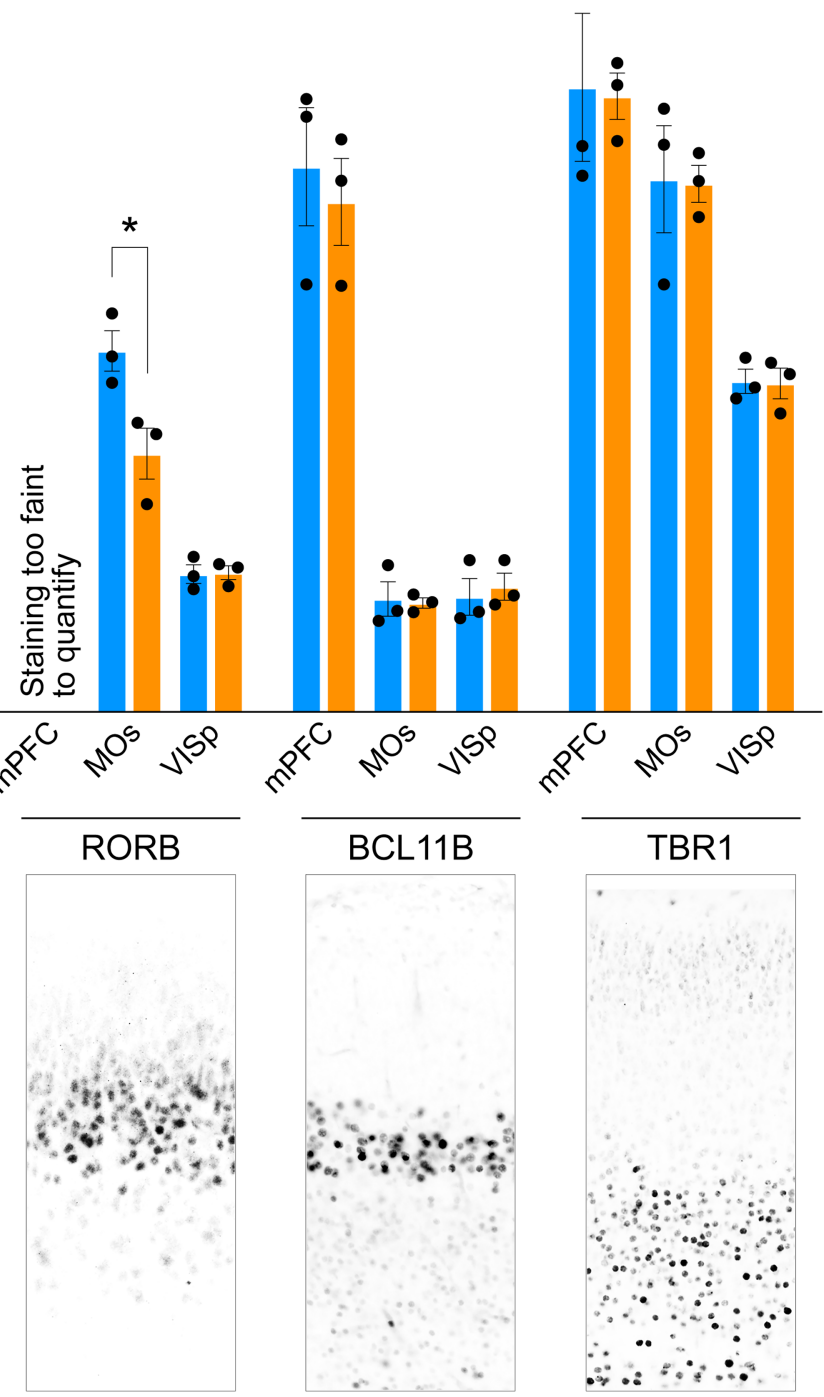

$P=0.3,0.1,0.6 . \mathrm{N}=5$. Errors bars: $\mathrm{S}$.E.M. b, Number of immune-positive cells expressing cortical layer markers POU3F2/BRN2, CUX1, RORB, BCL11B/CTIP2, TBR1 in WT and dKO mouse $\mathrm{MPFC}$, MOs, and primary visual cortex (VISp) at PD $5(\mathrm{~N}=3)$. (Unpaired t-test: WT vs. dKO ${ }^{*} P=0.03$; Errors bars: S.E.M.). RORB expression in mouse PFC was too faint to quantify. Below each graph is a representative image of each marker. Scale bar: $200 \mu \mathrm{m}$. 

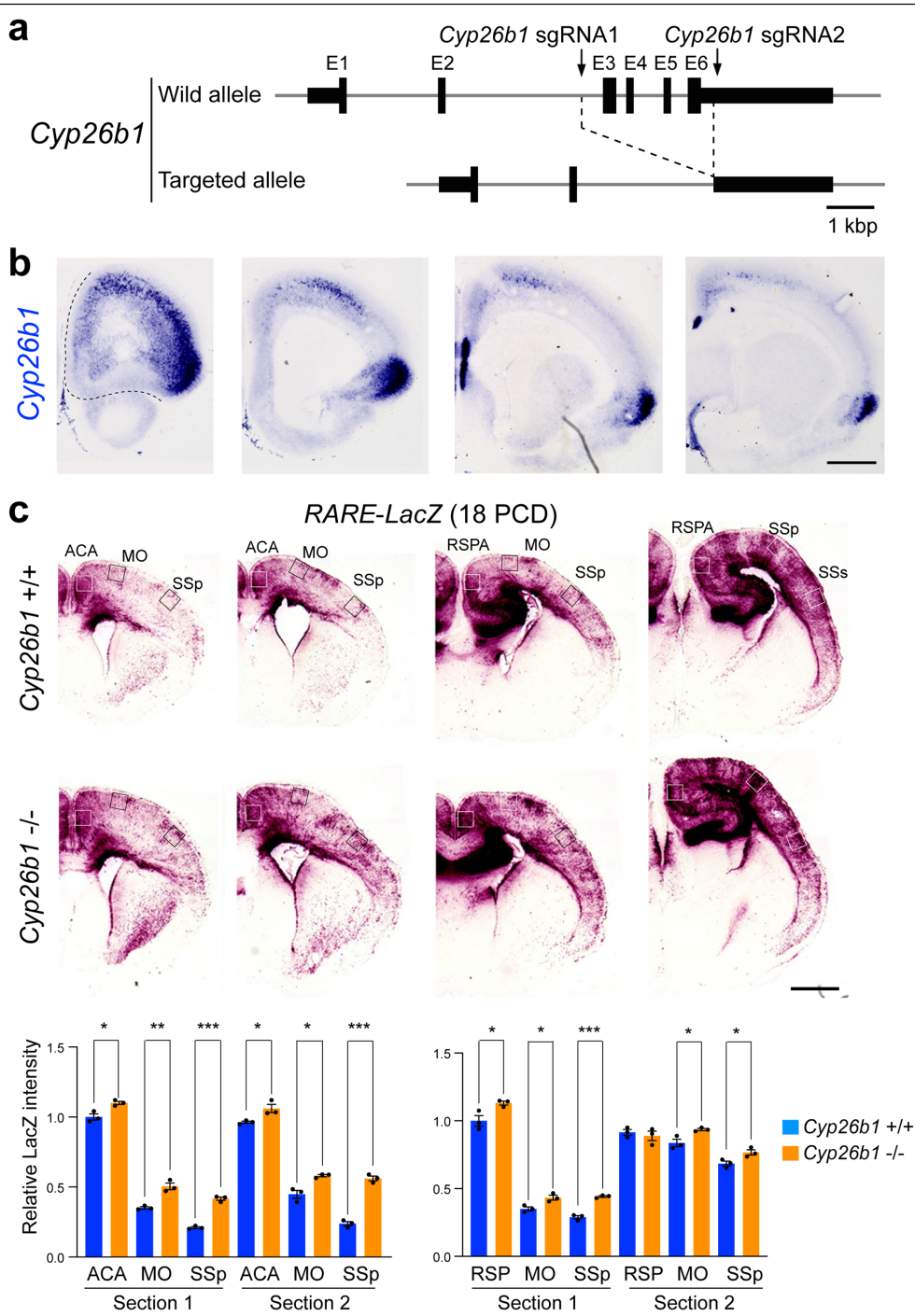

Extended Data Fig. 18 | RA signal in posterior cortical regions of Cyp26b1 KO mice. a, Strategy for the generation of Cyp26b1-- (Cyp26b1 KO) mice using CRISPR-Cas9 gene editing technique ${ }^{41}$.b, Cyp26b1 expression in PD 0 mice cortex by in situ hybridization. The colorimetric staining was purposefully extended compared to the experiment in Fig. 5 a to better visualize low control Cyp26b1+/+;RARE-lacZ (Ctrl) and Cyp26b1-/-;RARE-lacZ (KO) mouse brains at PCD 18. Scale bar: $500 \mu \mathrm{m}$. Intensity of signal in the boxed areas (ACA, $\mathrm{MO}, \mathrm{SSp}$ ) was quantified. Increase in RA signalling in Cyp26b1 KO brains is less significant in posterior regions. Two-tailed Student's t-test: Ctrl vs. Cyp26b1 KO: ${ }^{*} P=0.01 ; 0.04 ; 9 \mathrm{e}-3 ; 7 \mathrm{e}-3 ; 6 \mathrm{e}-3 ; 0.02 ; 0.02 ;{ }^{* *} \mathrm{P}=4 \mathrm{e}-3 ; 1 \mathrm{e}-3 ; 4 \mathrm{e}-3 ; 4 \mathrm{e}-3, \mathrm{~N}=3$ per genotype; Errors bars:S.E.M.;Scale bars: $200 \mu \mathrm{m}$. 
a

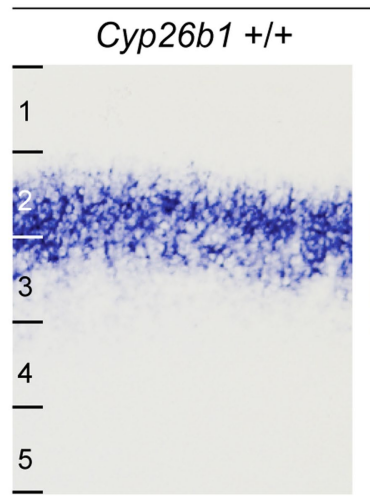

b

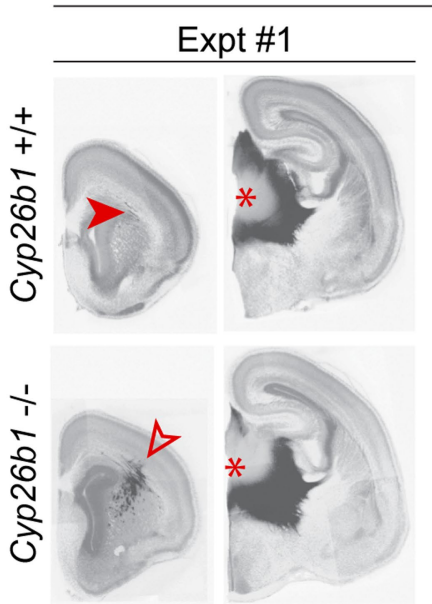

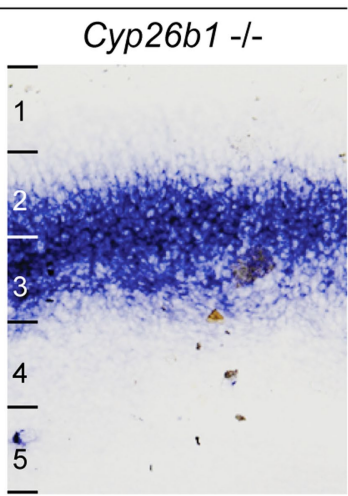

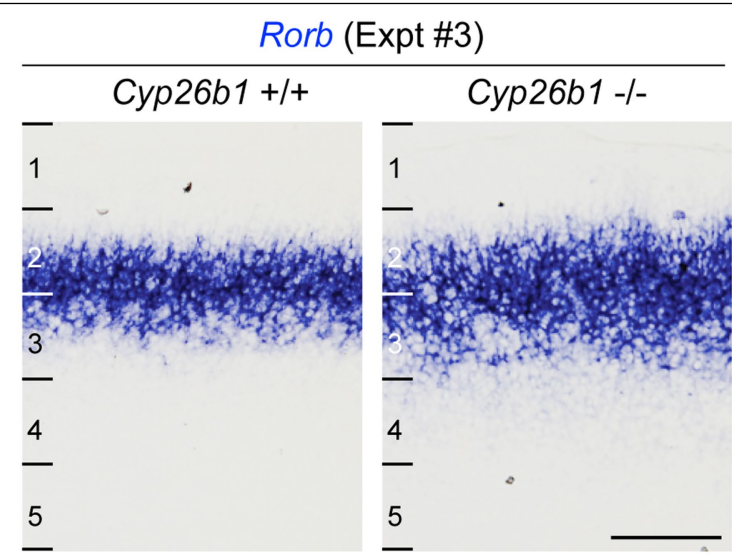

Dil placement in thalamus

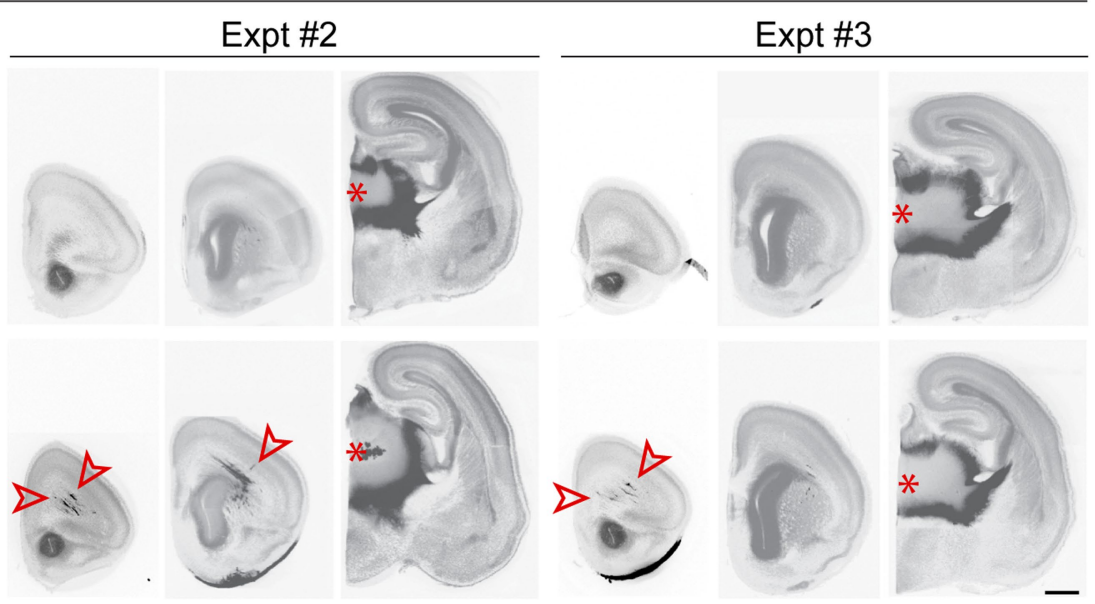

C

Dil placement in motor cortex

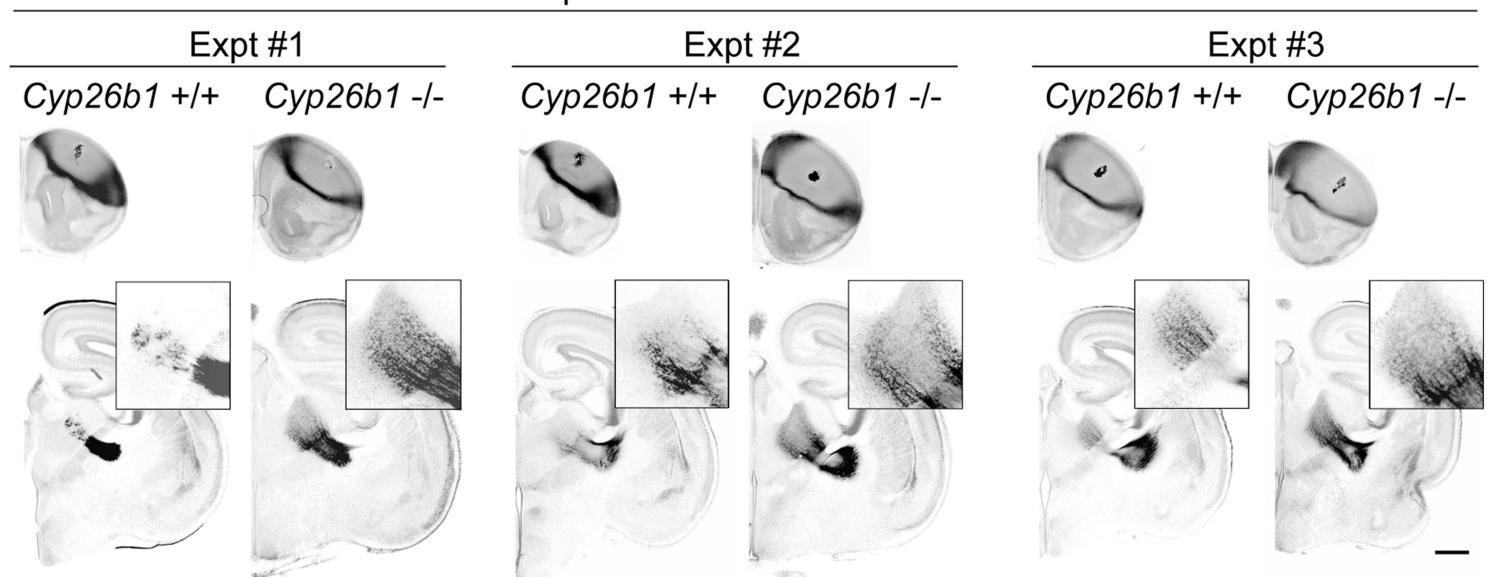

Extended Data Fig. 19|Rorb expression and medial thalamocortical projections in neonatal Cyp26b1 KO mice. a, Additional two replicates of Rorb expression in WT and Cyp26b1 KO brains from Fig. $5 \mathrm{f}$ are shown. Scale bar: $100 \mu \mathrm{m} . \mathbf{b}$, Dil was placed in the medial thalamus of WT and Cyp26b1 KO brains, and signal was detected in the PFC. Additional two replicates of experiment in Fig. $5 \mathrm{~d}$ are shown. $\mathrm{N}=3$ per genotype and condition. Arrowheads: Thalamocortical innervation of the medial and dorsolateral frontal cortex.
Asterisks: Dil crystals placed. Scale bar: $400 \mu \mathrm{m}$.c, Dil was placed in the frontal motor cortex of WT and Cyp26b1 KO brains, and signal was detected in the medial thalamus. Additional two replicates of experiment in Fig. 5e are shown. $\mathrm{N}=3$ per genotype and condition. Of note, owing to restriction related to Covid-19, PD 0 brains were left 2 months in cold room after 3 weeks in 37 degree. Scale bar: $400 \mu \mathrm{m}$. 
a

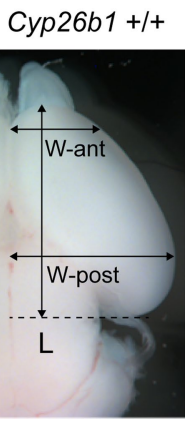

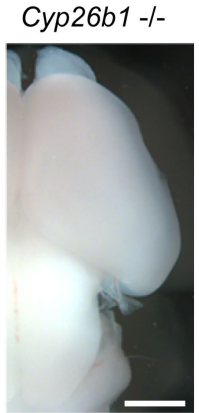

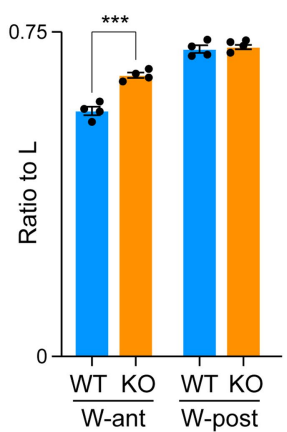

b
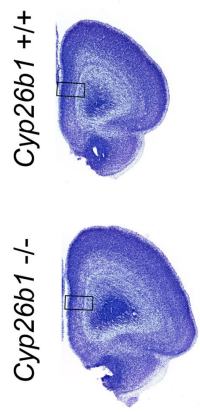

mPFC
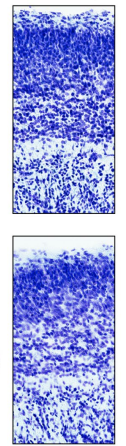
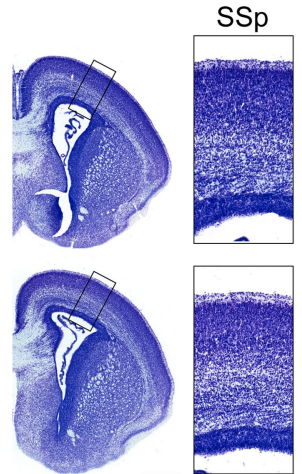

C pCAG-IRES-G $f p$

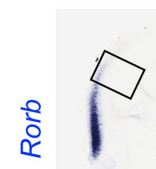

Electroporated side
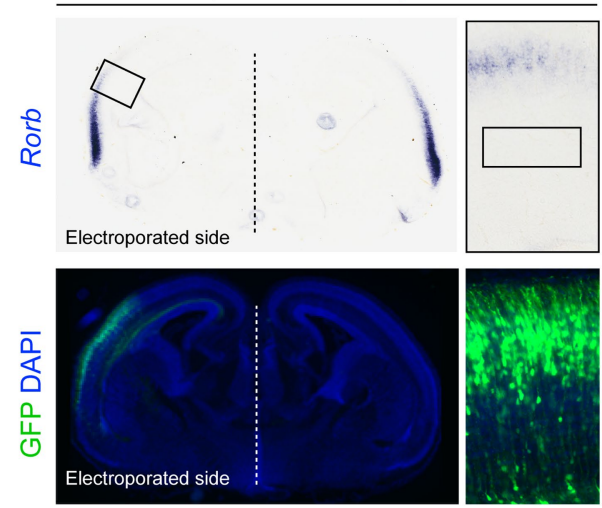

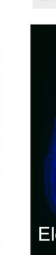

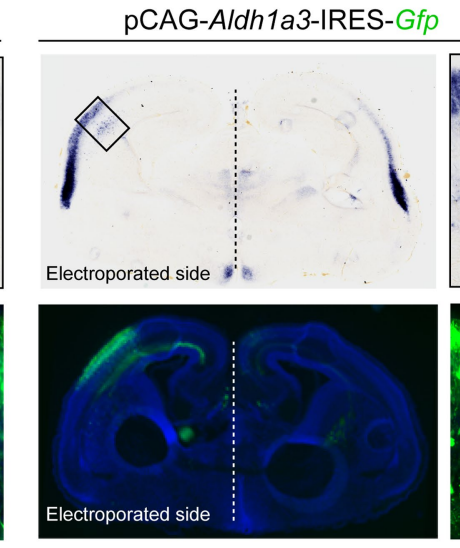

Extended Data Fig. 20 | Ectopic RA signalling leads to enlargement of the lateral region of the frontal cortex and upregulation of $R$ orb.

a, Representative image and quantification of the ratio of brain width at anterior and posterior cortex to total brain length at PCD 18 in Cyp26b1 KO brain compared WT. Two-tailed Student's t-test: WT vs. Cyp $26 b 1 \mathrm{KO}:^{* * *} P=3 \mathrm{e}-4$; $\mathrm{N}=4$ per genotype; Errors bars:S.E.M.b, Nissl staining reveals that the cortical wall and cortical plate are grossly normal when analysed in the MPFC and SSp of Cyp26b1 KO. $\mathrm{N}=3$ per genotype. $c$, Representative image and quantification of
Rorb expression after electroporation of either control pCAG-IRES-Gfp or pCAG-Aldh1a3-IRES-Gfp expression vector plasmid in the dorso-lateral fronto-parietal wall at PCD 14. Brains were dissected out at PD 5. Boxed region represents region of higher magnification to the right. GFP expression as a marker of misexpressing cells are shown in lower panels. Rorb signal intensity in the boxed area in the cortex was quantified. Two-tailed Student's t-test: ${ }^{* * *} \mathrm{P}=2 \mathrm{e}-4 ; \mathrm{N}=4$ per genotype; Errors bars: S.E.M.; Scale bars: $500 \mu \mathrm{m}(\mathbf{a})$; $200 \mu \mathrm{m}(\mathbf{b}) ; 1 \mathrm{~mm}(\mathbf{c}) ; 40 \mu \mathrm{m}$ and $500 \mu \mathrm{m}(\mathbf{d})$. 


\section{Reporting Summary}

Nature Research wishes to improve the reproducibility of the work that we publish. This form provides structure for consistency and transparency in reporting. For further information on Nature Research policies, see Authors \& Referees and the Editorial Policy Checklist.

\section{Statistical parameters}

When statistical analyses are reported, confirm that the following items are present in the relevant location (e.g. figure legend, table legend, main text, or Methods section).

n/a Confirmed

$\square \bigotimes$ The exact sample size $(n)$ for each experimental group/condition, given as a discrete number and unit of measurement

$\square$ An indication of whether measurements were taken from distinct samples or whether the same sample was measured repeatedly

$\square$ The statistical test(s) used AND whether they are one- or two-sided

Only common tests should be described solely by name; describe more complex techniques in the Methods section.

$\square \bigotimes$ A description of all covariates tested

$\square$ A description of any assumptions or corrections, such as tests of normality and adjustment for multiple comparisons

$\square$ A full description of the statistics including central tendency (e.g. means) or other basic estimates (e.g. regression coefficient) AND

variation (e.g. standard deviation) or associated estimates of uncertainty (e.g. confidence intervals)

$\varnothing$ For null hypothesis testing, the test statistic (e.g. $F, t, r$ ) with confidence intervals, effect sizes, degrees of freedom and $P$ value noted

Give $P$ values as exact values whenever suitable.

$\bigotimes \square$ For Bayesian analysis, information on the choice of priors and Markov chain Monte Carlo settings

Х $\square$ For hierarchical and complex designs, identification of the appropriate level for tests and full reporting of outcomes

\ $\square$ Estimates of effect sizes (e.g. Cohen's $d$, Pearson's $r$ ), indicating how they were calculated

$\varnothing$ Clearly defined error bars

State explicitly what error bars represent (e.g. SD, SE, CI)

Our web collection on statistics for biologists may be useful.

\section{Software and code}

Policy information about availability of computer code

Data collection No software was used for data collection.

Data analysis All software/code utilized for the analysis of data is publically available and described in the manuscript. Volocity (ver. 6.3.1), Spotfire (ver. 11.2.0) , Aperio ImageScope (ver. 12.4.3.5008), Reconstruct (ver. 1.1.0.0), ZEN2009, Adobe Photoshop (ver. 12.0 x64), Adobe Illustrator (ver. 23.1.1), and ImageJ (ver. 2.0.0-rc-69/1.52p) were used for basic image processing which included normalization by size and optical setting. MRtrix3 (ver. 3.0.0-65-g91788533) and ANTs (ver. 2.2.0.0.dev297-gf23cb) softwares were used for MRI images preprocessing. MRtrix3 (ver. 3.0.0-65-g91788533) was used for the brain segmentation and reconstruction of axonal pathways .

For manuscripts utilizing custom algorithms or software that are central to the research but not yet described in published literature, software must be made available to editors/reviewers upon request. We strongly encourage code deposition in a community repository (e.g. GitHub). See the Nature Research guidelines for submitting code \& software for further information. 
Policy information about availability of data

All manuscripts must include a data availability statement. This statement should provide the following information, where applicable:

- Accession codes, unique identifiers, or web links for publicly available datasets

- A list of figures that have associated raw data

- A description of any restrictions on data availability

Mouse RNA-seq data were deposited into the NCBI GEO database (Accession number GSE142851).

\section{Field-specific reporting}

Please select the best fit for your research. If you are not sure, read the appropriate sections before making your selection.

$\bigotimes$ Life sciences $\quad \square$ Behavioural \& social sciences $\quad \square$ Ecological, evolutionary \& environmental sciences

For a reference copy of the document with all sections, see nature.com/authors/policies/ReportingSummary-flat.pdf

\section{Life sciences study design}

All studies must disclose on these points even when the disclosure is negative.

Sample size No sample size calculation was performed. The size of the samples was determined by the availability of the samples, and the sequencing costs.

Data exclusions No data were excluded from the analysis.

Replication All mouse, macaque and human experiments performed in this manuscript with 3 biological replicates with the following exceptions: Human and macaque ALD1A1, ALD1A3, RARB, RXRG in situ hybridization experiments utilized 2 biological replicates (Fig. 2b; Extended Data Fig. 4f); MRI based Diffusion tensor imaging, scalar indexes and volumetric experiments utilized 5 biological replicates (Fig. 5b,c; Extended Data Fig. 16a,b; Extended Data Fig. 17a); ALDH1A3 Western blot experiments were replicated at least 2 times per species (Extended Data Fig. 4g); Human and chimpanzee organoid studies were performed on 3-5 replicate organoids per time and conditions (Extended Data Fig. 10a, b); Retrograde viral tracer experiments were performed with 4 biological replicates (Extended Data Fig.14a-c); Human primary cortical neuron experiments were performed with 8 fields per condition (Extended Data Fig. 15e-j); Electroporation experiments were performed with 4 biological replicates (Extended Data Fig. 20c); Quantification of mouse corticospinal tract (CST) width using L1CAM expression were performed with 5 biological replicates per genotype (Extended Data Fig. 16d); Quantification of apoptotic cells in the mouse mPFC detected by cleaved caspase3 were performed with 5 biological replicates per genotype (Extended Data Fig. 16e); Quantification of tyrosine hydroxylase (TH) immunolabeled axons in mouse frontal cortex were performed with 4 biological replicates per genotype (Extended Data Fig. 16f); Quantification of the ratio of brain width at anterior and posterior cortex to total brain length at PCD 18 were performed with 4 biological replicates per genotype (Extended Data Fig. 20a).

All experimental findings were reproducible.

Randomization Randomization was not relevant to this study as controls and mutant mouse lines did not receive different treatments, human/primate studies were descriptive studies, and human/chimpanzee organoids and human primary neuron experiments were generated using the same experimental conditions.

Blinding Group allocation was conducted as described above. Data collection was performed by independent investigators. Prior to data analysis, all experiments including immunohistological, histological, and MRI images and sequencing datasets were randomized and analyzed by independent, blinded observers as described in the methods section.

\section{Reporting for specific materials, systems and methods}


Materials \& experimental systems

\begin{tabular}{l}
\hline Involved in the study \\
$\square$ Unique biological materials \\
$\square$ Antibodies \\
$\square$ Eukaryotic cell lines \\
$\square$ Auman research participants
\end{tabular}

\section{Unique biological materials}

Policy information about availability of materials

Obtaining unique materials All unique materials used are readily available from the authors with proper MTA.

\section{Antibodies}

Antibodies used

Validation

anti-ALDH1A1 (1:500, Abcam, Cat. ab52490, anti-BCL11B/CTIP2 (1:500, Abcam, Cat. ab18465), anti-cleaved capase3 (1:500; Cell Signaling, Cat. 9611), anti-CUX1 (1:500, Santa Cruz Biotechnology, Cat. sc-13024), anti-GFAP (1:2000, Sigma-Aldrich, Cat. G3893), anti-GAD1 (1:50, R\&D systems, Cat. AF2086), anti-PAX6 (1:200; R\&D Systems, Cat. AF8150), anti-POU3F2/BRN2 (1:500, Sigma-Aldrich, Cat. SAB2702086), anti-DLG4/PSD95 (1:500; Invitrogen, Cat. 51-6900), anti-L1CAM (1:500; Millipore-Sigma, Cat. ABT143), anti-SATB2 (1:200, Abcam, Cat. ab92446), anti-SOX2 (1:200, R\&D Systems Cat. MAB2018), anti-TBR1 (1:200, Abcam, Cat. ab31940), anti-Tyrosine hydroxylase (1:1000, Immunostar, Cat. 22941), anti-TUBB3 (1:400; TuJ1 clone, Abcam, Cat.18207), Alexa Fluor 488-conjugated AffiniPureanti-IgG (1:200, Jackson ImmunoResearch, Cat. 715-545-150), Alexa Fluor 594-conjugated AffiniPure Donkey anti-IgG 1:200, (Jackson ImmunoResearch, Cat. 711-585-152), Alexa Fluor 647-conjugated AffiniPure Donkey anti-IgG (1:200, Jackson ImmunoResearch, Cat. 705-605-003), anti-GAPDH (1:5000, Abcam, Cat. ab9485), anti-ALDH1A3 (1:2000, Novus Biologicals, Cat. NBP2-15339), anti-RORB (1:500, Novus Biologicals, Cat. NBP1-82532), anti-NRGN (1:50, R\&D systems, Cat. AF7947), anti-NR4A2 (1:100, R\&D systems, Cat. AF2156), anti-SYP (1:2000, Sigma-Aldrich, Cat. SAB4200544), antiGFP antibody (1: 500, Abcam, Cat. Ab13970).

anti-ALDH1A1 (1:500, Abcam, Cat. ab52492, rabbit monoclonal IgG [EP1933Y], IHC, Jakob et al. 2021(PMID: 32588101)), antiBCL11B/CTIP2 (1:500, Abcam, Cat. ab18465, rat monoclonal IgG [25B6], IHC, Shim et al. 2012 (PMID: 22678282)), anti-cleaved capase3 (1:500; Cell Signaling, Cat. 9611, rabbit polyclonal IgG, IHC, Shim et al. 2012 (PMID: 22678282)), anti-CUX1 (1:500, Santa Cruz Biotechnology, Cat. sc-13024, rabbit polyclonal IgG, IHC, Shim et al. 2012 (PMID: 22678282)), anti-GFAP (1:2000, SigmaAldrich, Cat. G3893, mouse monoclonal IgG1 (G-A-5), IHC, Tebbencamp et al. 2018 (PMID: 30318146)), anti-GAD1 (1:50, R\&D systems, Cat. AF2086, Polyclonal Goat IgG, IHC, Sousa et al. 2017 (PMID: 29170230)), anti-PAX6 (1:200; R\&D Systems, Cat. AF8150, Polyclonal Sheep IgG, IHC, Shibata et al. 2011 (PMID: 21368052)), anti-POU3F2/BRN2 (1:500, Sigma-Aldrich, Cat. SAB2702086, rabbit polyclonal IgG, IHC, Shibata et al. 2011 (PMID: 21368052)), anti-DLG4/PSD95 (1:500; Invitrogen, Cat. 51-6900, rabbit polyclonal IgG, IHC,Velasco et al., 2019 (PMID: 31168097)), anti-L1CAM (1:500; Millipore-Sigma, Cat. ABT143, rabbit polyclonal IgG, IHC,Shim et al. 2012 (PMID: 22678282)), anti-SATB2 (1:200, Abcam, Cat. ab92446, rabbit monoclonal IgG (EPNCIR130A), IHC, Zhang et al. 2020 (PMID: 31666685)), anti-SOX2 (1:200, R\&D Systems Cat. MAB2018, Monoclonal Mouse IgG2A Clone \# 245610, IHC, Mikali et al., 2020 (PMID: 32375049)), anti-TBR1 (1:200, Abcam, Cat. ab31940, Rabbit polyclonal IgG, IHC, Han et al. 2011 (PMID: 21285371)), anti-Tyrosine hydroxylase (1:1000, Immunostar, Cat. 22941, Mouse Monoclonal IgG1, IHC, Sousa et al. 2017 (PMID: 29170230)), anti-TUBB3 (1:400; TuJ1 clone, Abcam, Cat.18207, rabbit polyclonal IgG, IHC, McCarthy N et al. 2020 (PMID: 32084389)), Alexa Fluor 488-conjugated AffiniPureanti-lgG (1:200, Jackson ImmunoResearch, Cat. 715-545-150, Donkey polyclonal IgG, IHC, Sousa et al. 2017 (PMID: 29170230)), Alexa Fluor 594-conjugated AffiniPure Donkey anti-IgG 1:200, (Jackson ImmunoResearch, Cat. 711-585-152, Donkey polyclonal IgG, IHC, Sousa et al. 2017 (PMID: 29170230)), Alexa Fluor 647-conjugated AffiniPure Donkey anti-IgG (1:200, Jackson ImmunoResearch, Cat. 705-605-003, Donkey polyclonal IgG, IHC, Sousa et al. 2017 (PMID: 29170230)), anti-GAPDH (1:5000, Abcam, Cat. ab9485, Rabbit polyclonal IgG, WB, Ni W et al. 2021 (PMID: 33376517)), anti-ALDH1A3 (1:2000, Novus Biologicals, Cat. NBP2-15339, rabbit polyclonal IgG, WB, Shrestha et al., 2020 (PMID: 32182217)), anti-RORB (1:500, Novus Biologicals, Cat. NBP1-82532, rabbit polyclonal IgG, IHC), antiNRGN (1:50, R\&D systems, Cat. AF7947, Polyclonal Sheep IgG, IHC, Tebbencamp et al.,2018 (PMID: 30318146)), anti-NR4A2 (1:100, R\&D systems, Cat. AF2156, Polyclonal Goat IgG, IHC, Sellnow et al., 2020 (PMID: 32238479)), anti-SYP (1:2000, SigmaAldrich, Cat. SAB4200544, mouse monoclonal SVP-38, IHC, D'Cruz et al., 2012 (PMID: 22970294)), anti-GFP antibody (1: 500, Abcam, Cat. Ab13970, Chicken polyclonal IgY, IHC, Shim et al. 2012 (PMID: 22678282))

\section{Eukaryotic cell lines}

Policy information about cell lines

Cell line source(s)

Human induced pluripotent stem cell line (cell line: HSB311 \#36), Chimpanzee induced pluripotent stem cell line (cell line 3651D), obtained from Yoav Gilad and Bryan Pavlovic (Department of Human Genetics, University of Chicago)

Authentication

Morphology or genotyping.

Mycoplasma contamination

All lines tested negative for mycoplasma contamination, checked monthly using the MycoAlert Mycoplasma Detection Kit (Lonza). 
Commonly misidentified lines (See ICLAC register)

\section{Animals and other organisms}

Policy information about studies involving animals; ARRIVE guidelines recommended for reporting animal research

Laboratory animals

Wild animals

Field-collected samples
B6SJLF1/J (The Jackson Laboratory) and CD-1 (Charles River laboratories) mice were used for the generation of Rarb, Rxrg and Cyp26b1 knockout mice. Knockout mice lines maintained on the C57BL6/6J (The Jackson Laboratory) background, were used at the following time points: E18 and postnatal day P0, P5, P21, and P30. Information on housing conditions for the mice was added in Method section. All relevant details of primate samples (Rhesus macaque and human) were described and put in the Source data file. Gender of mouse samples used for experiments was not specified in this study.

The study did not involve wild animals

The study did not involve samples collected from the wild.

\section{Human research participants}

Policy information about studies involving human research participants

Population characteristics Describe the covariate-relevant population characteristics of the human research participants (e.g. age, gender, genotypic information, past and current diagnosis and treatment categories). If you filled out the behavioural \& social sciences study design questions and have nothing to add here, write "See above."

Recruitment

Describe how participants were recruited. Outline any potential self-selection bias or other biases that may be present and how these are likely to impact results.

\section{Magnetic resonance imaging}

Experimental design

Design type

Design specifications

Behavioral performance measures

Ex vivo whole brains of wildtype controls and Rarb/Rxrg (dKO) mutant mice were imaged and analyzed

Acquisition

Imaging type(s)

Field strength

Sequence \& imaging parameters

Area of acquisition

Diffusion MRI

\Used

\section{Diffusion tensor imaging}

$$
9.4 \mathrm{~T}
$$

3D Stejskal-Tanner spin-echo sequence; $T R=2000 \mathrm{~ms} ; T R=25.616 \mathrm{~ms}$; in plane resolution $=0.11 \mathrm{~mm}$; slice thickness $=$ $0.22 \mathrm{~mm}$; orientation AP

Whole brain scan

Not used

Parameters 30 different angles of diffusion sensitization; $b$ value of $1000 \mathrm{~s} / \mathrm{mm} 2$; single shell; diffusion encoding duration $=4 \mathrm{~ms}$

\section{Preprocessing}

Preprocessing software

Normalization

Normalization template

Noise and artifact removal

Volume censoring

Statistical modeling \& inference

Model type and settings
Preprocessing was done with MRtrix3, software version: 3.0_RC3-135-g2b8e7d0c and ANTs, software version: 1.9

Data were not normalized since there are no normalization atlases for a specific KO group used in the study.

N/A

Denoising was done using the MRtrix denoise algorithm, and bias field corrections were done using corresponding ANTs' algorithm. Motion parameters and physiological signals were non-existed since the MRI was done ex-vivo.

N/A

N/A 
Effect(s) tested

Specify type of analysis:

Anatomical location(s) Thalamus and neocortex

Number of streamlines between the two groups

Statistic type for inference (See Eklund et al. 2016)

Two-tailed unpaired t-test or two tailed Mann-Whitney test

Correction

N/A

Models \& analysis

$\mathrm{n} / \mathrm{a} \mid$ Involved in the study

$\square \bigotimes$ Functional and/or effective connectivity

Х Graph analysis

Х $\square$ Multivariate modeling or predictive analysis

Functional and/or effective connectivity

Structural connectivity between brain regions was determined by the number of streamlines derived by seeding streamlines from a specific region of interest with an ending point in another region of interest. 